\title{
POWER ALLOCATION IN OFDM-BASED COGNITIVE RADIO SYSTEMS USING ITERATIVE ALGORITHMS
}

\author{
by \\ Aniqua Tasnim Rahman Antora \\ Bachelor of Science Degree in Electronic and Telecommunication Engineering \\ North South University, Bangladesh, 2013 \\ A Thesis \\ Presented to Ryerson University \\ in Partial Fulfillment of The \\ Requirements for The Degree of \\ Master of Applied Science \\ in \\ Electrical and Computer Engineering Program
}

Toronto, Ontario, Canada, 2015

(C)Aniqua Antora, 2015 


\title{
AUTHOR'S DECLARATION FOR ELECTRONIC SUBMISSION OF A THESIS
}

I hereby declare that I am the sole author of this thesis. This is a true copy of the thesis, including any required final revisions, as accepted by my examiners.

I authorize Ryerson University to lend this thesis to other institutions or individuals for the purpose of scholarly research

I further authorize Ryerson University to reproduce this thesis by photocopying or by other means, in total or in part, at the request of other institutions or individuals for the purpose of scholarly research.

I understand that my thesis may be made electronically available to the public.

\author{
Aniqua Antora
}


Power Allocation in OFDM-Based Cognitive Radio System Using Iterative Algorithms, Master of Applied Science, 2015, Aniqua Antora, Electrical and Computer Engineering Program with Specialization in Telecommunication Engineering, Ryerson University.

\section{Abstract}

As spectrum scarcity is becoming a serious problem, the worth of finding a general solution for such issue has become even serious due to the rapid development of wireless communications. The main objective of this thesis is to investigate the optimal power allocation procedure that maximizes the capacity in OFDM based Cognitive Radio Systems.

The main purpose of the search is to modify the conventional water-filling algorithm applied in general OFDM based Cognitive Radio systems due to the per subchannel power constraints and individual peak power constraints. For Radio Resource Allocation (RRA), one of the most typical problems is to solve power allocation using the Conventional Waterfilling. As communication system develops, the structures of the system models and the corresponding RRA problems evolve to more advanced and more complicated ones.

In this thesis Iterative Partitioned Weighted Geometric Water-filling with Individual Peak Power Constraints (IGPP), a simple and elegant approach is proposed to solve the weighted radio resource allocation problem with peak power constraint and total subchannel power constraint with channel partitions. The proposed IGPP algorithm requires less computation than the Conventional Water-filling algorithm (CWF).

Dynamic Channel Sensing Iterative (DCSI) approach is another algorithm proposed to optimally allocate power for OFDM based Cognitive Radio Systems. DCSI is a innovative concept which will allow us to solve the same problem intelligently with less complexity. It provides straight forward power allocation analysis, solutions and insights with reduced computation over other approaches under the same memory requirement and sorted parameters. 


\section{Acknowledgments}

I dedicate this study to my mother and my sister.

I wish to acknowledge those who supported me greatly in completing this thesis.

I would like to thank Professor Dr. Lian Zhao for providing an open study and discussion environment, having faith in me through out the project, constant encouragement and willingness to provide advice, and very clear guidance towards the success of this thesis project. I would also like to thank Dr. Pete He and Lilatul Ferdouse for their enlightening guidance, constructive suggestions, high-standard requirement, and unconditional support.

Special thanks to my colleagues, without their help I would not have been able to get through this difficult and emotional time. Special thanks to Hesam Nekouei, Salam Al-Juboori and Sagar Chandra Kar.

I am thankful for my supportive parents and grandparents. It is their blessing that has brought me so far. I would also like to thank my siblings for helping me cope with all sorts of situation, Specially Yosra Suzanne Rahman, who comforted me during hard times. At the end I would like to express appreciation to my better half, Mohammad Masurour Alam, who pushed me to strive towards my goal. 


\section{Contents}

1 Introduction 1

1.1 Spectrum Challenges . . . . . . . . . . . . . . . . . 1

1.2 Cognitive Radio . . . . . . . . . . . . . . . . . . . . 3

1.3 Orthogonal Frequency Division Multiplexing (OFDM) . . . . . . . . . 7

1.4 Thesis Motivation . . . . . . . . . . . . . . . . . . . . . 12

1.5 Research Contributions . . . . . . . . . . . . . . . . 13

1.6 Thesis Outline . . . . . . . . . . . . . . . . . . 13

2 Literature Review $\quad 15$

2.1 Water-Filling Method . . . . . . . . . . . . . . . . 15

2.1.1 Conventional Water-filling Method . . . . . . . . . . . . 17

2.1.2 Geometric Water-filling Method . . . . . . . . . . . . . . 19

2.1.3 Weighted Geometric Water-filling Method . . . . . . . . . . . . 22

2.1.4 Weighted Geometric Water-Filling with Individual Peak Power Con-

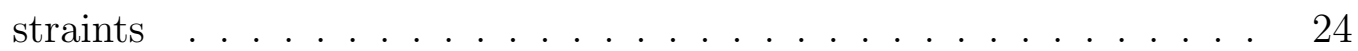

2.2 System Model . . . . . . . . . . . . . . . . . . . . 26

2.2.1 Iterative Partitioned Water-Filling Method . . . . . . . . . . . 30

2.3 Literature Survey . . . . . . . . . . . . . . . . . . . . 32

3 Algorithms and Procedures $\quad 41$

3.1 Problem Formulation . . . . . . . . . . . . . . . . . . 41 
3.2 Iterative Partitioned Weighted Geometric Water-filling with Individual Peak Power Constraints (IGPP) . . . . . . . . . . . . . . . . . . 42

3.2.1 IGPP Algorithm . . . . . . . . . . . . . . . . . 44

3.3 Dynamic Channel Sensing Iterative Method (DCSI) . . . . . . . . . . . . 50

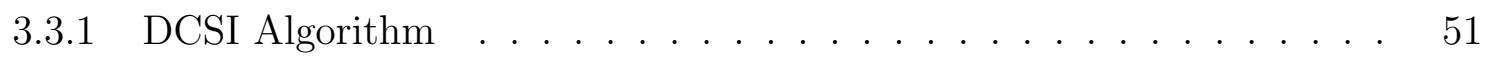

4 Numerical Results $\quad 59$

4.1 Results for Iterative Partitioned Weighted Geometric Water-filling with Individual Peak Power Constraints (IGPP) . . . . . . . . . . . . 59

4.2 Results for Dynamic Channel Sensing Iterative Method (DCSI) . . . . . . . 64

4.3 Comparison of CWF, IGPP and DCSI . . . . . . . . . . . . . . . 69

5 Conclusion and Future Work $\quad 75$ 


\section{List of Tables}

4.1 Calculation Comparison of $\left\{P_{i}\right\}$ using IPW, GWFPP and IGPP based on the numerical results captured from Fig. 4.2 to Fig. 4.4 . . . . . . . . . . . . . 64

4.2 Calculation Comparison of $\left\{P_{i}\right\}$ using IPW, GWFPP and DCSI based on the numerical results captured from Fig. 4.7 to Fig. 4.9 . . . . . . . . . . . 68 


\section{List of Figures}

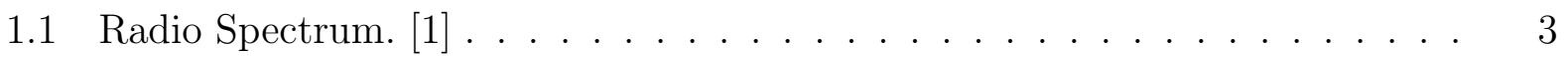

1.2 Different Types of Spectrum Sensing Methodologies to improve Spectrum Utilization in Different Domain. [2] . . . . . . . . . . . . . . . 4

1.3 Cognitive Radio Concept Architecture. [3] . . . . . . . . . . . . . . 5

1.4 Orthogonal Frequency Division Multiplexing (OFDM). . . . . . . . . 8

1.5 Orthogonal Frequency Division Multiplexing Spectral Efficiency [4]. . . . . . 9

1.6 Orthogonal Frequency Division Multiplexing (OFDM) Architecture [4]. . . . 10

2.1 Classical Water-Filling Power Allocation $P_{i}=\left(\mu-h_{i}^{-1}\right)^{+}$with $\sum_{i} P_{i}=P_{t} \quad$. 16

2.2 General Water-Filling Power Allocation $P_{i}=\left(\mu-h_{i}^{-1}\right)^{+}$with $\sum_{i} P_{i} w_{i}=P_{t} . \quad 17$

2.3 Illustration for the proposed Geometric Water-Filling (GWF) algorithm. (a) Illustration of water level step $n^{*}=3$, allocated power for the third step $P_{3}^{*}$, and step/stair depth $d_{i}=1 / h_{i}$. (b) Illustration of $P_{t}(n)$ (shadowed area, representing the total water/power above step $n$ ) when $n=2$. (c) Illustration of $P_{t}(n)$ when $n=3$. (d) Illustration of the weighted case. . . . . . . . . . 20

2.4 Cognitive Radio System Model . . . . . . . . . . . . . . . . . 27

2.5 Spectrum of Secondary User in OFDM-Based Cognitive Radio Systems . . . 28

3.1 Block Diagram of IGPP . . . . . . . . . . . . . . . . . . . . . . 47

3.2 Illustration for IPW, GWFPP and the proposed Iterative Partitioned Weighted Geometric Water-filling with Individual Peak Power Constraints (IGPP). . . 48

3.3 Block Diagram of DCSI . . . . . . . . . . . . . . . . 53

3.4 Channel Fading . . . . . . . . . . . . . . . . . . . . . . . . . . . 54 
3.5 Illustration of DCSI Algorithm in Subchannel $1 \ldots \ldots$. . . . . . . . 55

3.6 Illustration of DCSI Algorithm in Subchannel $3 \ldots \ldots$. . . . . . . 56

3.7 Illustration of DCSI Algorithm in Subchannel $2 \ldots \ldots$. . . . . . . . 57

3.8 Illustration of DCSI Algorithm in all the Subcarriers . . . . . . . . . 58

4.1 Parameters Used For The Following Experiment . . . . . . . . . . . . . 60

4.2 Iterative Partitioned Water-filling Method . . . . . . . . . . . . 60

4.3 Geometric Water-filling Method with Peak Power Constraints . . . . . . . . 61

4.4 Power allocation using Iterative Partitioned Weighted Geometric Water-filling with Individual Peak Power Constraints (IGPP) Algorithm . . . . . . . . . . 62

4.5 Optimal Power Allocation vs Subcarriers for IPW, GWFPP and IGPP . . . 63

4.6 Parameters Used For The Following Experiment . . . . . . . . . . . . . 65

4.7 Iterative Partitioned Water-filling Method _ . . . . . . . . . . . 65

4.8 Geometric Water-filling Method with Peak Power Constraints . . . . . . . 66

4.9 Power Allocation Using Dynamic Channel Sensing Iterative Method _ . . 67

4.10 Optimal Power Allocation vs Subcarriers for IPW, GWFPP and DCSI . . 67

4.11 Conventional Water-filling Method _. . . . . . . . . . . . 70

4.12 Parameters Used For The Following Experiment . . . . . . . . . . . . 70

4.13 Iterative Partitioned Water-filling Method . . . . . . . . . . . . 71

4.14 Geometric Water-filling with Individual Peak Power Constraints . . . . . . . 71

4.15 Power allocation using Iterative Partitioned Weighted Geometric Water-filling with Individual Peak Power Constraints (IGPP) Algorithm . . . . . . . . 72

4.16 Power Allocation using Dynamic Channel Sensing Iterative (DCSI) Algorithm 73

4.17 Comparison of Power Allocation with Different Algorithm. . . . . . . . . . 73 


\section{Chapter 1}

\section{Introduction}

Radio Frequency (RF) and wireless have been around for over a century with Alexander Popov and Sir Oliver Lodge laying the groundwork for Guglielmo Marconis wireless radio developments in the early 20th century. In December 1901, Marconi performed his most prominent experiment, where he successfully transmitted Morse code from Cornwall, England, to St Johns, Canada. Today, radio communications has become a highly sophisticated field of electronics.

\subsection{Spectrum Challenges}

The Radio Frequency (RF) Spectrum is a limited natural resource, which is governed by the laws of physics. Theoretically, the RF commences from approximately $9 \mathrm{KHz}$ and extends up to $3000 \mathrm{GHz}$. However, in practical scenario, not every part of the spectrum is suitable for being used for all requirements.

Mobile communication is feasible up to approx. $6 \mathrm{GHz}$ band, with currently available technology. Even within this practically usable spectrum as per current available technologies, cost effective equipment for a particular application may be available for use in still smaller/ limited frequency bands. This is the practical limitation on the spectrum due to propagation and availability of suitable equipment. 
The Canadian Table of Frequency Allocations assigns the electromagnetic spectrum and establishes the frequency allocations available for radio services in Canada. The Canadian Table is based on the provisions of the Final Acts resulting from the various World Radiocommunication Conferences (WRC), which are convened by the International Telecommunication Union (ITU). This spectrum allocation policy refers to command- and control mechanism in which the Government may decide the following: ration spectrum, specify technologies and services for spectrum use, put in strict mergers and acquisition (M\&A) norms, and confer non-sharable rights to spectrum holders etc. [5]. This static spectrum allocation mechanism causes frequency bands to be insufficient in various times and locations.

Fig. 1.1 [6] illustrates the radio spectrum and the broad range of frequencies in wireless communication context. Most of the fractions of the radio spectrum are licensed to traditional radio communications systems. Beside, practical measurements, prove that most of the licensed bands either are unused or partially used at different geographical areas at most of the times. According to the FCCs report the licensed spectrum band utilization range from $15 \%$ to $85 \%$ at different times and locations [7].

While the number of wireless connections and high data rate networks increase, spectrum demand and spectrum congestion become critical challenges in the forthcoming allencompassing wireless world. In fact, throughput, high reliability, high quality of service, mobility, and diversity of wireless services, devices based on multiple wireless standards are more and more demanded. Hence, future wireless networks will face greater spectrum scarcity due to the users requirement such as high multimedia data rate transmission and diversity of communication technologies.

The under utilization of some frequency bands opens up the opportunity to identify and exploit spectrum holes. A spectrum hole is defined as a band of frequencies assigned to a primary user $(P U)$, but, at a particular time and specific geographic location, the band is not being utilized by that user [8]. If a secondary user $(S U)$ can access a spectrum hole, the 


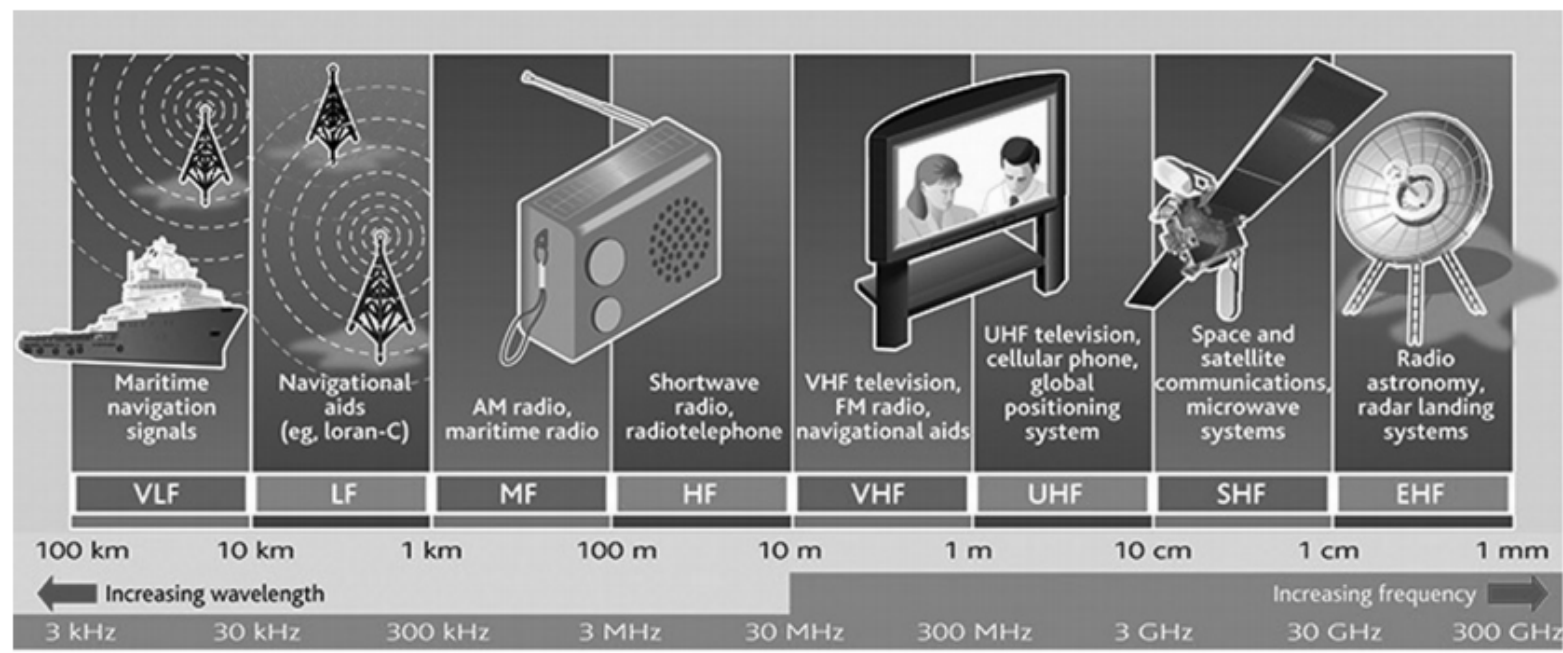

Figure 1.1: Radio Spectrum. [1]

spectrum utilization is improved significantly.

\subsection{Cognitive Radio}

The considerations mentioned previously have motivated the search for breakthrough radio technologies that can scale to meet future demands both in terms of spectrum efficiency and application performance. A promising mechanism to improve the spectrum utilization by exploiting the spectrum holes is based on the cognitive radio concept.

The definition adopted by Federal Communications Commission (FCC): Cognitive radio: A radio or system that senses its operational electromagnetic environment and can dynamically and autonomously adjust its radio operating parameters to modify system operation, such as maximize throughput, mitigate interference, facilitate interpretability, access secondary markets [9].

Fig. 1.2 shows some of the spectrum sensing option used to improve spectrum utilization. 


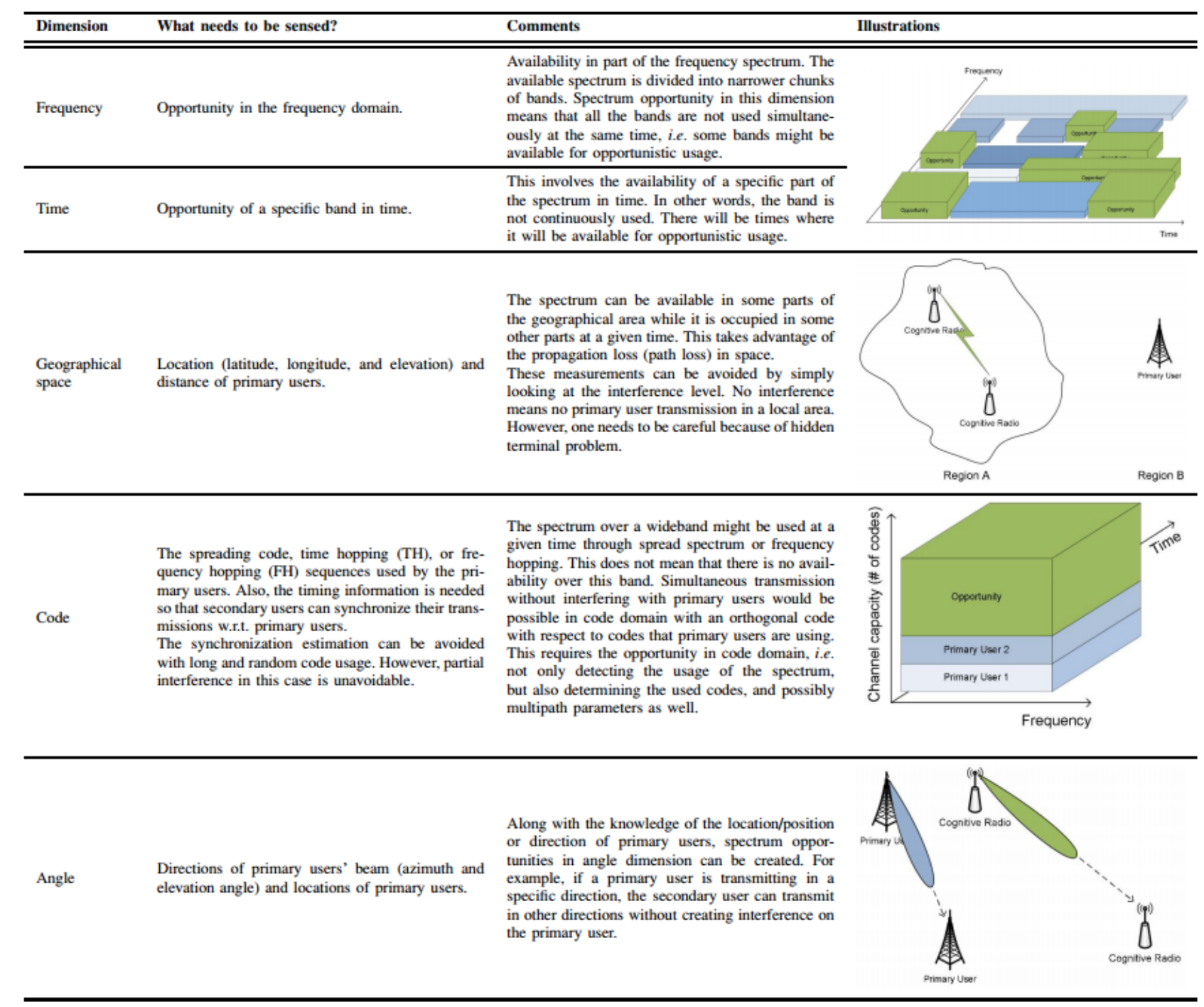

Figure 1.2: Different Types of Spectrum Sensing Methodologies to improve Spectrum Utilization in Different Domain. [2]

One of the most important components of the cognitive radio concept is the ability to measure, sense, learn, and be aware of the parameters related to the radio channel characteristics, availability of spectrum and power, radios operating environment, user requirements and applications, available networks and nodes, local policies and other operating restrictions.

Cognitive Radio Concept Architecture: There are two major subsystems in a cognitive radio: 
- A cognitive unit that makes decisions based on various inputs.

- A flexible SDR unit whose operating software provides a range of possible operating modes.

A separate spectrum sensing subsystem is also often included in the architecture of cognitive radio to measure the signal environment to determine the presence of other services or users. It is important to note that these subsystems do not necessarily define a single piece of equipment, but may instead incorporate components that are spread across an entire network. As a result, cognitive radio is often referred to as a cognitive radio system or a cognitive network.

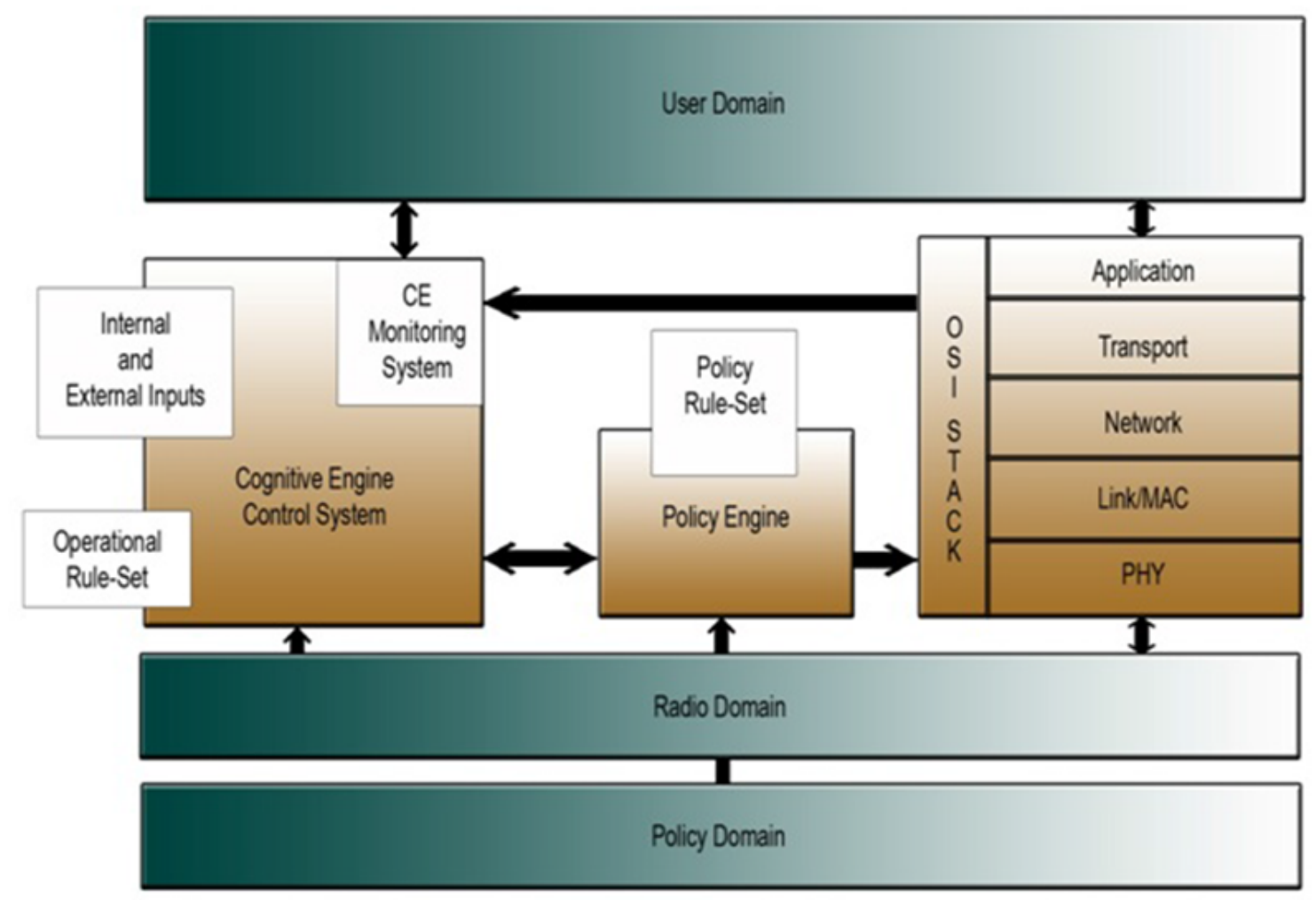

Figure 1.3: Cognitive Radio Concept Architecture. [3]

The cognitive unit is further separated into two parts as shown in the Fig. 1.3. They are: 
- The first labeled the Cognitive Engine tries to find a solution or optimize a performance goal based on the inputs received defining the radios current internal state and operating environment.

- The second engine is the Policy Engine and is used to ensure that the solution provided by the Cognitive Engine is in compliance with the regulatory rules and other policies external to the radio.

Primary Users: These wireless devices are the primary license-holders of the spectrum band of interest. In general, they have priority accessing the spectrum, and subject to certain Quality of Service (QoS) constraints which must be guaranteed.

Secondary Users: These users may access the spectrum which is licensed to the primary users. They are thus secondary users of the wireless spectrum, and are often envisioned to be cognitive radios.

Cognitive Radio (CR) is an adaptive, intelligent radio and network technology that can automatically detect available spectrum and change transmission parameters enabling more communications to run concurrently and also improve radio operating behavior.

The Importance of Cognitive Radio Networks: Cognitive networks are motivated by the apparent lack of spectrum under the current spectrum management policies. The right to use the wireless spectrum in the United States is controlled by the Federal Communications Commission (FCC) [9]. Most of the frequency bands useful to wireless communication have already been licensed by the FCC [10]. A few bands have however been designated by the FCC to be unlicensed bands, most notably the Industrial Scientific and Medical bands (ISM bands), over which the immensely popular WiFi devices transmit. These bands are filling up fast, and despite their popularity, the vast majority of the wireless spectrum is in fact licensed. 
Currently, the primary license holders obtain from the FCC the exclusive right to transmit over their spectral bands. As most of the bands have been licensed out, and the unlicensed bands are also rapidly filling up, it would appear that we are approaching a spectral crisis. This, however, is far from the case.

Recent measurements in [11] have shown that for as much as $90 \%$ of the time, large portions of the licensed bands remain unused. As licensed bands are difficult to reclaim and re-lease, the FCC is considering dynamic and secondary spectrum licensing [9] [10] as an alternative to reduce the amount of unused spectrum. Bands licensed to primary users could, under certain negotiable conditions, be shared with non-primary users without having the primary licensee release its own license. Whether the primary users would be willing to share their spectrum would depend on a number of factors, including the impact on their own communication.

Cognitive radios, wireless devices with reconfigurable hardware and software (including transmission parameters and protocols) [12], are capable of delivering what these secondary devices would need: the ability to intelligently sense and adapt to their spectral environment. Along with this newfound flexibility comes the challenge of understanding the limits of and designing protocols and transmission schemes to fully exploit these cognitive capabilities.

In particular, in order to design practical and efficient protocols, the theoretical limits must be well understood. We next describe different scenarios, assumptions and corresponding types of cognitive behavior, for which information theoretic limits have been considered [3].

\subsection{Orthogonal Frequency Division Multiplexing (OFDM)}

Frequency Division Multiplexing (FDM) extends the concept of single carrier modulation by using multiple subcarriers within the same single channel. The total data rate to be 
sent in the channel is divided between the various subcarriers. The data do not have to be divided evenly nor do they have to originate from the same information source. Advantages include using separate modulation/demodulation customized to a particular type of data, or sending out banks of dissimilar data that can be best sent using multiple, and possibly different, modulation schemes.

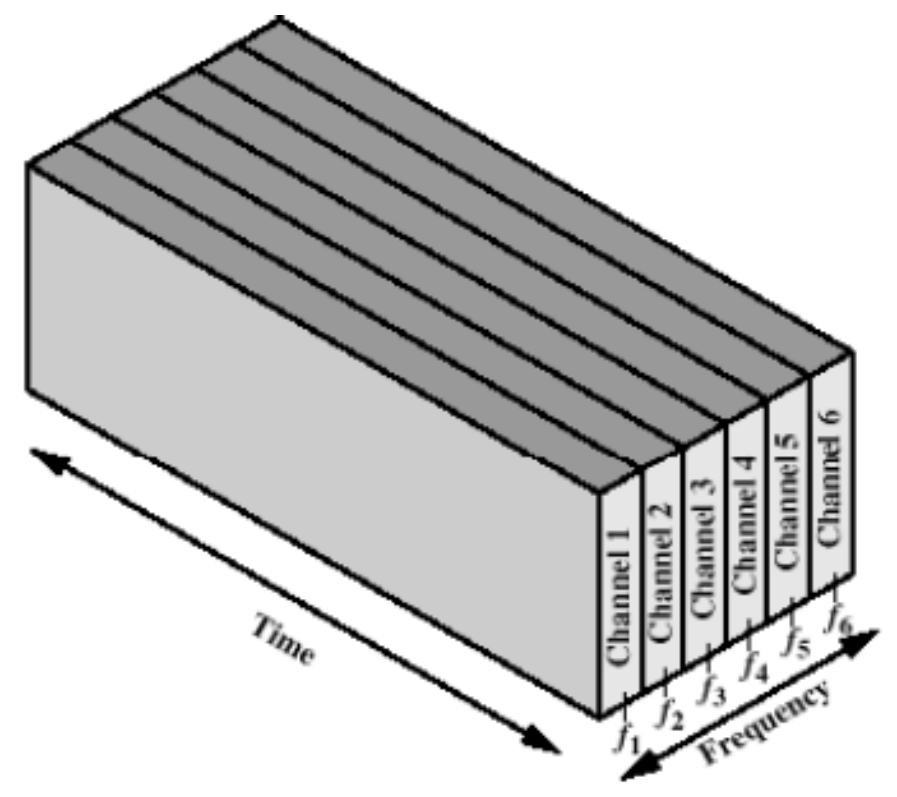

Figure 1.4: Orthogonal Frequency Division Multiplexing (OFDM).

Conceptually, OFDM is a specialized Frequency Division Multiplexing, the additional constraint being: all the carrier signals are orthogonal to each other.

In OFDM, the sub-carrier frequencies are chosen so that the sub-carriers are orthogonal to each other, meaning that cross-talk between the sub-channels is eliminated and inter-carrier guard bands are not required. This greatly simplifies the design of both the transmitter and the receiver; unlike conventional FDM, a separate filter for each sub-channel is not required.

Fig. 1.5 shows the comparison of OFDM networks with $2 \mathrm{G}$ and $3 \mathrm{G}$ networks. From the diagram we can reach a conclusion that OFDM is a preferred physical layer access technology over other methods. 


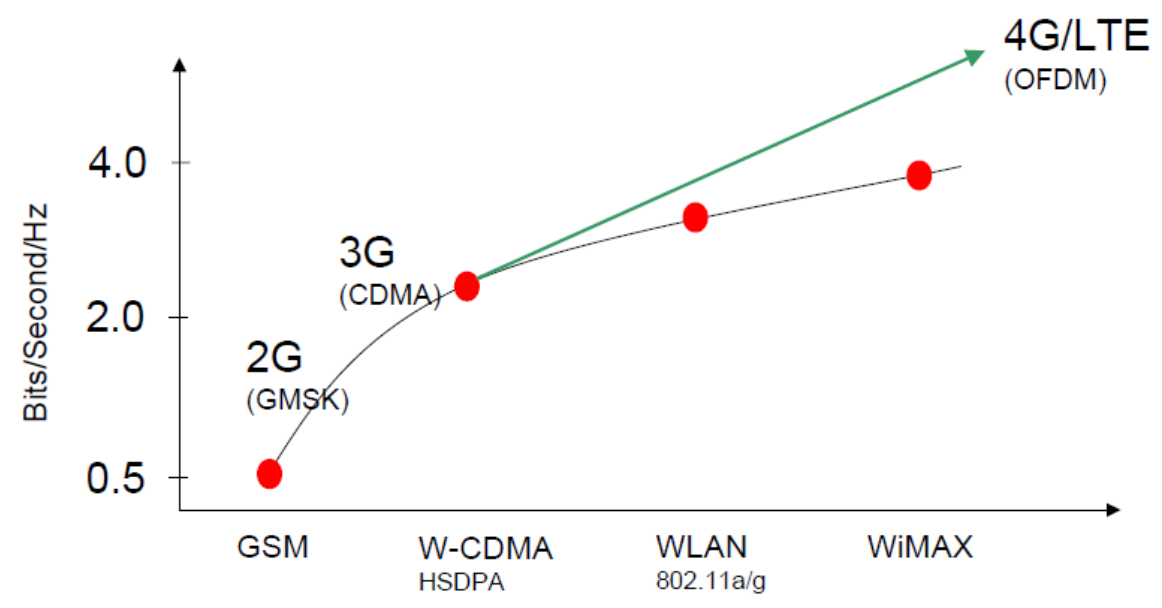

Figure 1.5: Orthogonal Frequency Division Multiplexing Spectral Efficiency [4].

OFDM Architecture: The idea behind the analog implementation of OFDM can be extended to the digital domain by using the Discrete Fourier Transform (DFT) and its counterpart, the Inverse Discrete Fourier Transform (IDFT). These mathematical operations are widely used for transforming data between the time-domain and frequency-domain. These transforms are interesting from the OFDM perspective because they can be viewed as mapping data onto orthogonal subcarriers.

For example, the IDFT is used to take in frequency-domain data and convert it to timedomain data. In order to perform that operation, the IDFT correlates the frequency-domain input data with its orthogonal basis functions, which are sinusoids at certain frequencies. This correlation is equivalent to mapping the input data onto the sinusoidal basis functions $[4]$.

In practice, OFDM systems are implemented using a combination of Fast Fourier Transform (FFT) and Inverse Fast Fourier Transform (IFFT) blocks that are mathematically equivalent versions of the DFT and IDFT, respectively, but more efficient to implement. An OFDM system treats the source symbols (e.g., the QPSK or QAM symbols that would be present in a single carrier system) at the transmitter as though they are in the frequency- 


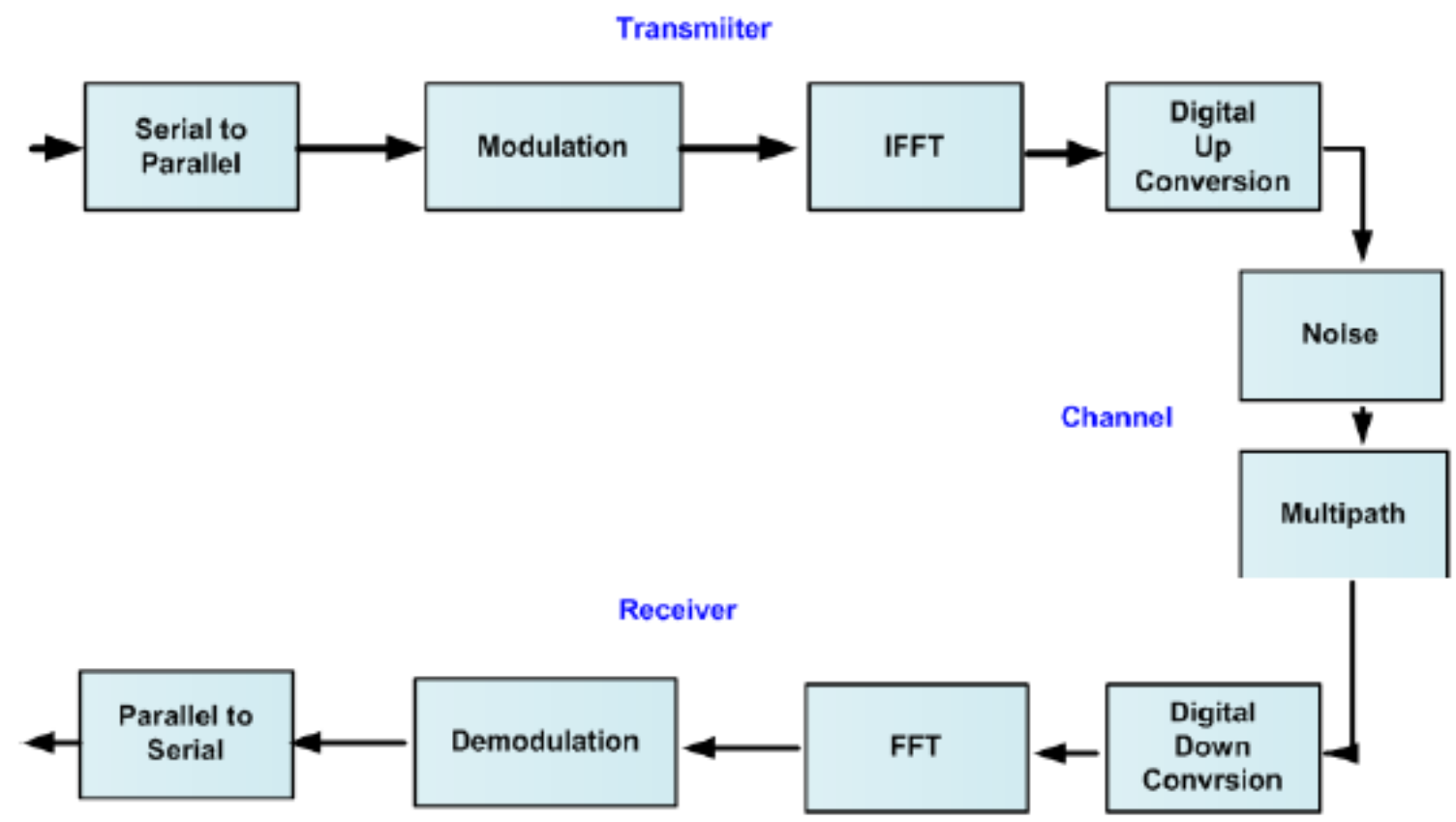

Figure 1.6: Orthogonal Frequency Division Multiplexing (OFDM) Architecture [4].

domain [4].

These symbols are used as the inputs to an IFFT block that brings the signal into the time domain. The IFFT takes in $N$ symbols at a time where $N$ is the number of subcarriers in the system. Each of these $N$ input symbols has a symbol period of $T$ seconds. Recall that the basic functions for an IFFT are $N$ orthogonal sinusoids. These sinusoids each have a different frequency. Each input symbol acts like a complex weight for the corresponding sinusoidal basis function. Since the input symbols are complex, the value of the symbol determines both the amplitude and phase of the sinusoid for that subcarrier. The IFFT output is the summation of all $N$ sinusoids. Thus, the IFFT block provides a simple way to modulate data onto $N$ orthogonal subcarriers [4]. 
The block of $N$ output samples from the IFFT make up a single OFDM symbol. The length of the OFDM symbol is $N T$ where $T$ is the IFFT input symbol period mentioned above. After some additional processing, the time-domain signal that results from the IFFT is transmitted across the channel. At the receiver, an FFT block is used to process the received signal and bring it into the frequency domain. Ideally, the FFT output will be the original symbols that were sent to the IFFT at the transmitter. When plotted in the complex plane, the FFT output samples will form a constellation, such as $16-Q A M$ [4].

However, there is no notion of a constellation for the time-domain signal. When plotted on the complex plane, the time-domain signal forms a scatter plot with no regular shape. Thus, any receiver processing that uses the concept of a constellation (such as symbol slicing) must occur in the frequency- domain [4].

OFDM Advantages: OFDM has been used in many high data rate wireless systems because of the many advantages it provides [13].

- One of the main advantages of OFDM is that is more resistant to frequency selective fading than single carrier systems because it divides the overall channel into multiple narrow band signals that are affected individually as flat fading sub-channels.

- Interference appearing on a channel may be bandwidth limited and in this way will not affect all the sub-channels. This means that not all the data is lost.

- Using close-spaced overlapping sub-carriers, a significant OFDM advantage is that it makes efficient use of the available spectrum.

- Another advantage of OFDM is that it is very resilient to inter-symbol and inter-frame interference. This results from the low data rate on each of the sub-channels.

- Using adequate channel coding and interleaving it is possible to recover symbols lost due to the frequency selectivity of the channel and narrow band interference. Not all the data is lost. 
- One of the issues with CDMA systems was the complexity of the channel equalisation which had to be applied across the whole channel. An advantage of OFDM is that using multiple sub-channels, the channel equalization becomes much simpler.

\subsection{Thesis Motivation}

As spectrum scarcity is becoming a serious problem, the worth of finding a general solution for such issue has become even serious due to the rapid development of wireless communications.

In [14] the power allocation problem in OFDM-based cognitive radio systems is modeled based on the convex optimization theory. the authors present and prove the sufficient and necessary conditions that the optimal power allocation should satisfy; compared to an intuitive algorithm, a more efficient algorithm, named as Iterative Partitioned Water-Filling (IPW) is proposed and proved to be optimal.

In [15] a simple and elegant Geometric Water-Filling (GWF) approach is proposed to solve the weighted radio resource allocation problems. Geometric Water-Filling Peak to Peak Power Constraints (GWFPP) Algorithm is a dynamic power distribution process. The state of this process is the difference between the individual peak power sequence and the current power distribution sequence obtained by the Algorithm GWF [15].

In this thesis, we proposed two efficient power allocation algorithms for OFDM-based cognitive radio networks with low computational complexity. By exploiting some properties of the Iterative Partitioned Water-Filling and Geometric Water-Filling Peak to Peak Power Constraints method, we proposed Iterative Partitioned Weighted Geometric Water-filling with Individual Peak Power Constraints (IGPP) and Dynamic Channel Sensing Iterative 
Method (DCSI) to solve the target problem. Thus, the algorithm can be used to solve the power allocation problem with high efficiency.

\subsection{Research Contributions}

In this paper we utilize both [14] and [15] algorithms to come up with Iterative Partitioned Weighted Geometric Water-filling with Individual Peak Power Constraints (IGPP), an unique solution which maximizes the power allocation to the $S U$ s better than if algorithm [14] and algorithm [15] were to run separately. In this thesis we have also introduced Dynamic Channel Sensing Iterative Method (DCSI) algorithm which is a combined recursive algorithm efficiently allocating power to the SUs.

The key contributions of this thesis to the field of Power Allocation in OFDM-based Cognitive Radio System is as follows:

- Proposing a new hybrid algorithm which uses Iterative Partitioned Weighted Peak Power Geometric Water-Filling Method.

- Proposing another new iterative algorithm called Dynamic Channel Sensing Iterative Method which uses Channel Sensing Information.

- Evaluating the proposed algorithms and comparing with other algorithms and illustrating improved performance.

\subsection{Thesis Outline}

The remaining chapters of this thesis are structured as follows: 
Chapter 2: Literature Review. Introduce background information, water-filling algorithm as a fundamental tool in radio resource management and review literature to present the state of the art technologies in the area.

Chapter 3: Algorithms and Procedures. Present an overview of the related approaches to the algorithm proposed in this thesis. Describe the methods which we use for the proposed new algorithms. Present the proposed algorithms IGPP and DCSI. Illustrate the proposed algorithms in details.

Chapter 4: Numerical Results. Numerical results of the IGPP and DCSI are presented in this chapter. Compare the proposed algorithm results with the related work results. Demonstrate the amount of improvement based on practical parameters.

Chapter 5: Conclusion and Future Work. Conclude the thesis, and propose future work for improvement. 


\section{Chapter 2}

\section{Literature Review}

In this Chapter, we first discuss the different types of Water-Filling (WF) methods like: Conventional Water-Filling, Geometric Water-Filling Method (GWF), Weighted Geometric Water-Filling Method (WGWF), Weighted Water-Filling with Individual Peak Power Constraints (WFPP) and Iterative Partitioned Water-Filling Method. Then we have literature review to report related work in the open literature.

\subsection{Water-Filling Method}

The well-known classical water-filling solution solves the problem of maximizing the mutual information between the input and the output of a channel composed of several subchannels (such as a frequency-selective channel, a time-varying channel, or a set of parallel subchannels arising from the use of multiple antennas at both sides of the link) with a global power constraint at the transmitter [16].

In traditional OFDM systems, given a certain power constraint, the optimal power allocation maximizing the sum capacity is the well-known water-filling algorithm [17].

Many engineering problems that can be formulated as constrained optimization problems result in solutions given by a water-filling structure. This capacity-achieving solution has 
the visual interpretation of pouring water into a tank whose bottom is given by the inverse of the subchannel gains, hence the name water-filling or water-pouring [16].

In Fig. 2.1, this capacity-achieving solution has the visual interpretation of pouring water over a surface (or curve) given by the inverse of the subchannel gains, hence, the name waterfilling or waterpouring [18], [19]. More general water-filling expressions such as $P_{i}=\left(\mu a_{i}-d_{i}\right)^{+}$, where the $a_{i}$ 's and $d_{i}$ 's are arbitrary positive numbers, also have the same visual interpretation after the change of variables $\tilde{P}_{i}=P_{i} / a_{i}, h_{i}=a_{i} / d_{i}$ and with a resulting weighted power constraint given by $\sum_{i} \tilde{P}_{i} w_{i}=P_{t}$, where $w_{i}=a_{i}$ are weights that can be visually interpreted as the width of each of the subchannels as can be observed from Fig. $2.2[16]$.

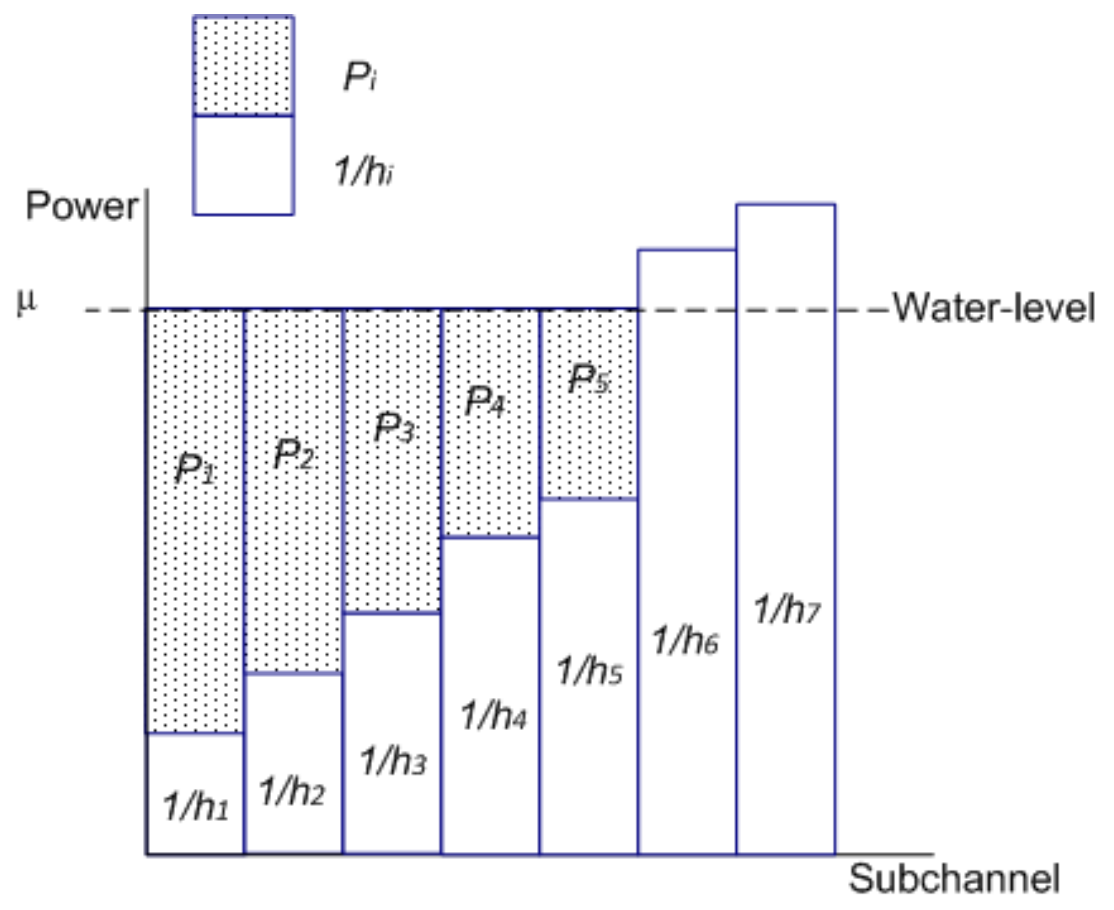

Figure 2.1: Classical Water-Filling Power Allocation $P_{i}=\left(\mu-h_{i}^{-1}\right)^{+}$with $\sum_{i} P_{i}=P_{t}$ 


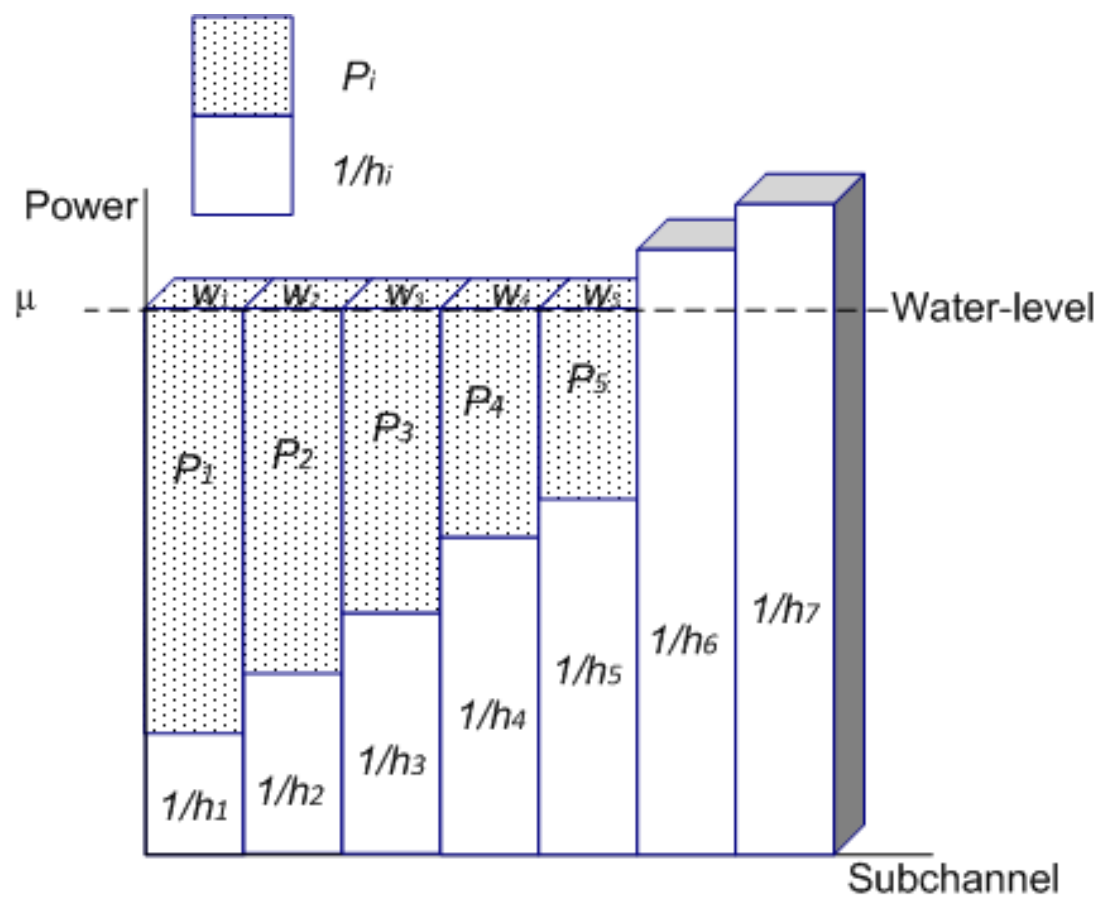

Figure 2.2: General Water-Filling Power Allocation $P_{i}=\left(\mu-h_{i}^{-1}\right)^{+}$with $\sum_{i} P_{i} w_{i}=P_{t}$

Many different constrained optimization problems result in water-filling solutions. In order to obtain an algorithm to evaluate a specific water-filling solution, it suffices to particularize a general algorithm. Such an algorithm indeed gives the desired solution with a linear worstcase complexity.

\subsubsection{Conventional Water-filling Method}

The conventional water-filling (CWF) problem has a sum power constraint under nonnegative individual powers. Since the solution is parameterized with a water level, the problem reduces to obtaining the water level such that the power constraint is satisfied with equality. This leads to a non-linear system, in one parameter, such as the water level, that is determined by the sum power constraint and the function itself. Further, this non-linear system consists of a non-linear equation and another inequality to find the water level. 
A class of methods to solve the non-linear system are called the conventional WF algorithm. In order to find the exact value of the water level, different algorithms have been proposed that can be classified into iterative algorithms and exact algorithms.

The conventional water-filling problem can be abstracted and generalized into the following problem: given $P_{t}>0$, as the total power or volume of the water; the allocated power and the propagation path gain for the $i$ th channel are given as $P_{i}$ and $h_{i}$ respectively, $i=1 \ldots N$; and $N$ is the total number of subcarriers. Let $\left\{h_{i}\right\}_{i=1}^{N}$ be a sorted sequence, which is positive and monotonically decreasing, find that

$$
\begin{array}{ll}
\max _{\left\{P_{i}\right\}_{i=1}^{N}} & \sum_{i=1}^{N} \log \left(1+h_{i} P_{i}\right) \\
\text { subject to: } & 0 \leq P_{i}, \forall i \\
& \sum_{i=1}^{N} P_{i}=P_{t}
\end{array}
$$

To find the solution to problem (2.1), we usually start from the Karush-Kuhn-Tucker (KKT) conditions of the problem, as a group of the optimality conditions, and derive the system (2.2) below from the KKT conditions,

$$
\left\{\begin{array}{l}
P_{i}=\left(\mu-\frac{1}{h_{i}}\right)^{+}, \text {for } i=1, \ldots, N \\
\sum_{i=1}^{N} P_{i}=P_{t} \\
\mu \geq 0
\end{array}\right.
$$

where $(x)^{+}=\max \{0, x\} . \mu$ is the water level chosen to satisfy the power sum constraints with equality $\left(\sum_{i=1}^{N} P_{i}=P_{t}\right)$. The solution to $(2.2)$ is referred as a solution of the CWF problem (2.1).

It can be seen that the implied system (2.2) has been used to find the optimal solution. The existence of its Lagrange multipliers and the implication mentioned above determine that enumeration can be utilized to find the water level $\mu$. 


\subsubsection{Geometric Water-filling Method}

In paper [15] a geometric water-filling (GWF) approach is proposed to solve the conventional waterfilling problem and its weighted form. It has two advantages, they are:

1) The geometric approach can compute the exact solution to the CWF, including the weighted case, with less computation and easier analysis without determining the water level through solving the non-linear system.

2) Machinery of the proposed geometric approach can overcome the limitations of the CWF algorithm to include more stringent constraints.

Figure 2.3 gives an illustration of the Gemetric Water-Filling (GWF) algorithm proposed in [15]. Suppose there are 4 steps/stairs $(N=4)$ with unit width inside a water tank. For the conventional approach, the dashed horizontal line, which is the water level $\mu$, needs to be determined first and then the power allocated (water volume) above is solved.

Let us use $d_{i}$ to denote the "step depth" of the $i$ th stair which is the height of the $i$ th step to the bottom of the tank, and is given as

$$
d_{i}=\frac{1}{h_{i}}, \text { for } i=1,2, \ldots, N .
$$

Since the sequence $h_{i}$ is sorted as monotonically decreasing, the step depth of the stairs indexed as $[1, \cdots, N]$ is monotonically increasing. We further define $\delta_{i, j}$ as the "step depth difference" of the $i$ th and the $j$ th stairs, expressed as,

$$
\delta_{i, j}=d_{i}-d_{j}=1 / h_{i}-1 / h_{j} \text {, as } i \geq j \text { and } 1 \leq i, j \leq N .
$$

Instead of trying to determine the water level $\mu$, which is a real nonnegative number, the water level step is the target to solve. The water level step is an integer number from 1 to $N$, denoted by $n^{*}$, as the highest step under water. Based on the result of $n^{*}$, we can write out the solutions for power allocation instantly. 


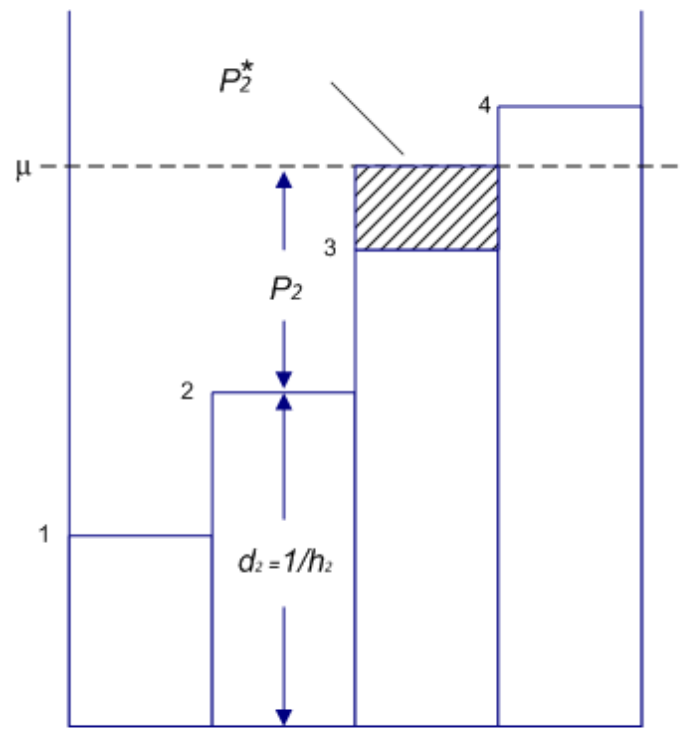

(a)

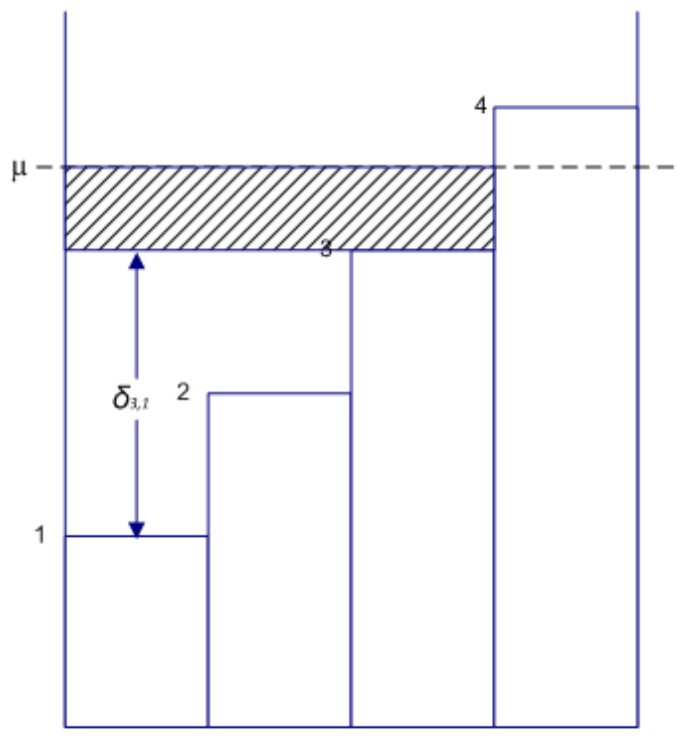

(c)

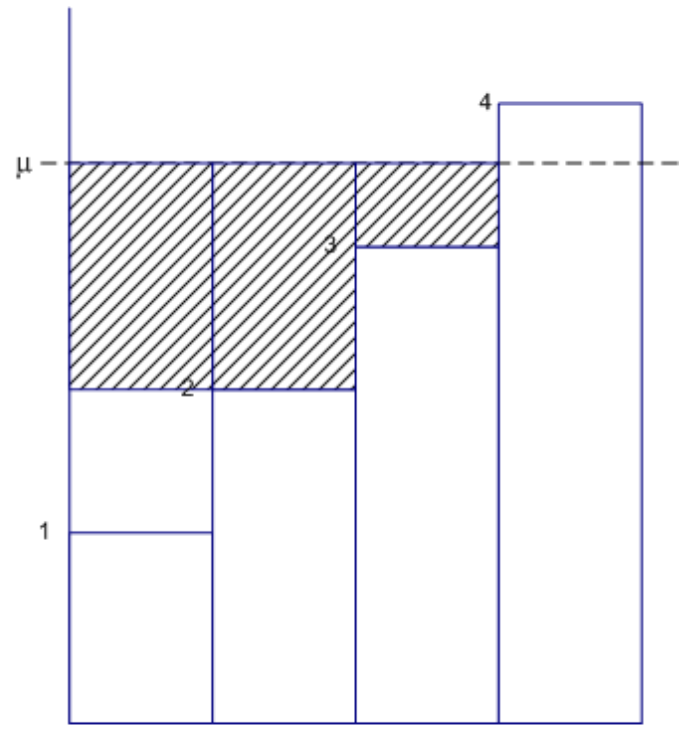

(b)

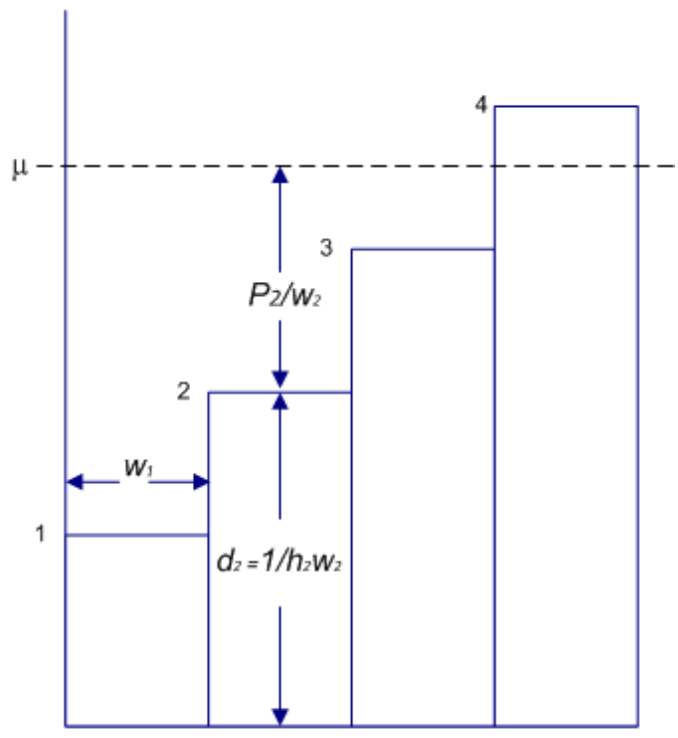

(d)

Figure 2.3: Illustration for the proposed Geometric Water-Filling (GWF) algorithm. (a) Illustration of water level step $n^{*}=3$, allocated power for the third step $P_{3}^{*}$, and step/stair depth $d_{i}=1 / h_{i}$. (b) Illustration of $P_{t}(n)$ (shadowed area, representing the total water/power above step $n$ ) when $n=2$. (c) Illustration of $P_{t}(n)$ when $n=3$. (d) Illustration of the weighted case.

Figure 2.3 illustrates the concept of $n^{*}$. Since the third level is the highest level under water, we have $n^{*}=3$. The shaded area denotes the allocated power for the third step by $P_{3}^{*}$. 
In the following, we explain how to find the water level step $n^{*}$ without the knowledge of the water level $\mu$. Let $P_{t}(n)$ denote the water volume above step $n$ or zero, whichever is greater. The value of $P_{t}(n)$ can be solved by subtracting the volume of the water under step $n$ from the total power $P_{t}$, as,

$$
\begin{aligned}
P_{t}(n) & =\left\{P_{t}-\left[\sum_{i=1}^{n-1}\left(\frac{1}{h_{n}}-\frac{1}{h_{i}}\right)\right]\right\}^{+} \\
& =\left\{P_{t}-\left[\sum_{i=1}^{n-1} \delta_{n, i}\right]\right\}^{+}, \text {for } n=1, \ldots, N
\end{aligned}
$$

Due to the definition of $P_{t}(n)$ being the power (water volume) above step $n$, it cann't be a negative number. Therefore we use $\{\cdot\}^{+}$in (2.5) to assign 0 to $P_{t}(n)$ if the result inside the bracket is negative. The corresponding geometric meaning is that the $n$th level is above water.

The above result is the shadowed area in Fig. 2.3, which is also an expansion of the composite form of (2.5).

Then the following proposition was proposed:

The explicit solution to (2.1) is:

$$
P_{i}= \begin{cases}P_{n^{*}}+\left(d_{n^{*}}-d_{i}\right) & 1 \leq i \leq n^{*} \\ 0, & n^{*}<i \leq N,\end{cases}
$$

where the water level step $n^{*}$ is given as

$$
n^{*}=\max \left\{n \mid P_{t}(n)>0, \quad 1 \leq n \leq N\right\}
$$

and the power level for this step is

$$
P_{n^{*}}=\frac{1}{n^{*}} P_{t}\left(n^{*}\right)
$$




\subsubsection{Weighted Geometric Water-filling Method}

For the weighted case, the generalized problem can be stated as: given $P_{t}>0$, as the total power or volume of the water; the allocated power and the propagation path gain for the $i$ th antenna are given as $P_{i}$ and $h_{i}$ respectively, $i=1, \ldots, N$; and $N$ is the total number of the transmit antenna. Furthermore, the weighted coefficients $w_{i}>0, i \in[0, N]$, and $\left\{h_{i} w_{i}\right\}_{i=1}^{N}$ being monotonically decreasing, such that

$$
\begin{array}{ll}
\max _{\left\{P_{i}\right\}_{i=1}^{N}} & \sum_{i=1}^{N} w_{i} \log \left(1+h_{i} P_{i}\right) \\
\text { subject to: } & 0 \leq P_{i}, \forall i \\
& \sum_{i=1}^{N} P_{i}=P_{t}
\end{array}
$$

Using the proposed geometric approach, the geometric relation for the weighted case can be extended as shown in Fig. (2.3) to obtain the solution.

In Fig. (2.3), the width of the $i$ th step is denoted as $w_{i}$. The value of $1 / h_{i}$ denotes the volume under the $i$ th step to the bottom of the tank. Hence, the step depth of the $i$ th step is given as,

$$
d_{i}=1 /\left(h_{i} w_{i}\right), \quad i=1, \cdots, N \text {. }
$$

Then, $P_{t}(n)$, the water volume above step $n$, can be obtained using the similar approach as in the previous subsection considering the step depth difference and the width of the stairs as,

$$
\begin{aligned}
& P_{t}(n)=\left[P_{t}-\sum_{i=1}^{n-1}\left(d_{n}-d_{i}\right) w_{i}\right]^{+}, \text {for } \\
& n=1, \ldots, N
\end{aligned}
$$

As an example in Fig. (2.3), the water volume above step 1 and below step 3 with the width $w_{1}$ can be found as: the step depth difference, $\left(d_{3}-d_{1}\right)$ multiplying the width of the step, $w_{1}$. Therefore, the corresponding $P_{t}(n=3)$ can be expressed as,

$$
P_{t}(n=3)=\left[P_{t}-\left(d_{3}-d_{1}\right) w_{1}-\left(d_{3}-d_{2}\right) w_{2}\right]^{+},
$$


which is an expansion of (2.11). The explicit solution to (2.9) is:

$$
\begin{cases}P_{i}=\left[P_{n^{*}} / w_{n^{*}}+\left(d_{n^{*}}-d_{i}\right)\right] w_{i}, & 1 \leq i \leq n^{*} \\ P_{i}=0, & n^{*} \leq i \leq N\end{cases}
$$

where,

$$
n^{*}=\max \left\{n \mid P_{t}(n)>0,1 \leq n \leq N\right\}
$$

and the power level for this step is,

$$
P_{n^{*}}=\frac{w_{n^{*}}}{\sum_{i=1}^{n^{*}} w_{i}} P_{t}\left(n^{*}\right)
$$

Similar to the unweighted case, the first step is to calculate $P_{t}(n)$, then find the water level step, $n^{*}$, from (2.14), which is the maximal index making $P_{t}(n)$ nonnegative. The corresponding power level for this step, $P_{n^{*}}$, can be obtained by applying (2.15). Then for those steps with index higher than $n^{*}$, the power level is assigned with zero. For those steps below $n^{*}$, the power level is assigned as in (2.14).

With the GWF approach, the weighted problem could be solved straightforwardly, avoiding complicated derivation and calculation. When the weighting factors are set to ones, the corresponding unweighted case is obtained.

In the following description of algorithm implementation, we only provide weighted case.

When $n^{*}$ is obtained, $P_{t}\left(n^{*}\right)$ is given.

Then it is memorized and only multiplied by a constant to compute $P_{n^{*}}$. Thus, how to search $n^{*}$ is a key point for the proposed GWF and the procedure is stated as follows:

1) Initialize $W_{P}=0 ; P_{t_{M}}=P_{t}^{*}=P_{t} ; i=1$. 
2) Compute $W_{P}<=W_{P}+w_{i} ; P_{t}^{*}<=P_{t}^{*}-\left(d_{i+1}-d_{i}\right) W_{P}$. Then $i<=i+1$, where the symbol " $<=$ " represents the assignment operation.

3) If $P_{t}^{*}>0$ and $i \leq N, P_{t_{M}}=P_{t}^{*}$, and repeat the step 2 ; else, output $n^{*}=i-1$ and $P_{n^{*}}=\frac{w_{n^{*}}}{W_{P}} P_{t_{M}}$

It is easy to see that $\frac{P_{n^{*}}}{w_{n^{*}}}+d_{n^{*}}$ is the water level due to $\frac{P_{n^{*}}}{w_{n^{*}}}+d_{n^{*}}=\frac{P_{i}}{w_{i}}+d_{i}$, for $1 \leq i \leq n^{*}$.

\subsubsection{Weighted Geometric Water-Filling with Individual Peak Power Constraints}

The weighted WFPP problem is stated as follows. Given $P_{t}>0$, as the total power or volume of the water; the allocated power and the propagation path gain for the $i$ th antenna are given as $P_{i}$ and $h_{i}$ respectively, $i=1, \ldots, N$; and $N$ is the total number of the transmit antenna. Also, the weights $w_{i}>0, \forall i$, and without loss of generality, $\left\{h_{i} \cdot w_{i}\right\}_{i=1}^{N}$ being positive and monotonically decreasing, find that,

$$
\begin{array}{ll}
\max _{\left\{P_{i}\right\}_{i=1}^{N}} & \sum_{i=1}^{N} w_{i} \log \left(1+h_{i} P_{i}\right) \\
\text { subject to: } & 0 \leq P_{i} \leq S_{i}, \forall i \\
& \sum_{i=1}^{N} P_{i} \leq P_{t}
\end{array}
$$

Compare the problem (2.16) with (2.9), the constraint of $0 \leq P_{i}$ is extended to $0 \leq P_{i} \leq S_{i}$, i.e., additional individual peak power constraints, and $\sum_{i=1}^{N} P_{i}=P_{t}$ to $\sum_{i=1}^{N} P_{i} \leq P_{t}$.

The problem (2.16) is thus referred to as (weighted) water-filling with sum and individual peak power constraints (WFPP). In this section, we discuss the solution to the WFPP problem.

The following presents an algorithm which is a modification of the above discussed GWF and it is termed as the GWFPP. 
For convenience, the expression (2.11) can be extended into the expression:

$$
\begin{aligned}
& P_{t}\left(i_{n}\right)=\left[P_{t}-\sum_{t=1}^{|E|-1}\left(\frac{1}{d_{i_{n}}}-\frac{1}{d_{i_{t}}}\right) w_{i_{t}}\right]^{+}, \\
& \text {for } n=1, \ldots,|E|
\end{aligned}
$$

where $E$ is a subsequence of the sequence $\{1,2, \ldots, P\},|E|$ is the cardinality of the set $E$, so $E$ can be expressed as $\left\{i_{1}, i_{2}, \ldots, i_{|E|}\right\}$. Especially, if $E$ is taken as the sequence $\{1,2, \ldots, N\}$, then the extended expression is regressed into the original expression.

Algorithm GWFPP: Input: vector $\left\{d_{i}\right\},\left\{w_{i}\right\},\left\{P_{t_{i}}\right\}$ for $i=1,2, \ldots, N$, the set $E=$ $\{1,2, \ldots, N\}$, and $P_{t}$.

1) utilize (2.14)-(2.15)compute $\left\{P_{i}\right\}$.

2) The set $\Lambda$ is defined by the set $\left\{i \mid P_{i}>P_{t_{i}}, i \in E\right\}$. If $\Lambda$ is the empty set, output $\left\{P_{i}\right\}_{i=1}^{N}$; else, $P_{i}=P_{t_{i}}$, as $i \in \Lambda$.

3) Update $E$ with $E \backslash \Lambda$ and $P_{t}$ with $P_{t}-\sum_{t \in \Lambda} P_{t}$. Then return to 1$)$ of the GWFPP.

Algorithm GWFPP is a dynamic power distribution process. The state of this process is the difference between the individual peak power sequence and the current power distribution sequence obtained by the Algorithm GWF. The control of this process is to use (2.14)-(2.15) of the Algorithm GWF based on the state mentioned above. Thus, a new state for next time stage appears. Therefore, an optimal dynamic power distribution process, the GWFPP, with the state feedback is formed. Since the finite set $E$ is getting smaller and smaller until the set $\Lambda$ is empty, Algorithm GWFPP carries out $N$ loops to compute the optimal solution, at most. 


\subsection{System Model}

The terms Cognitive Radio and Orthogonal Frequency Division Multiplexing can be understood better, with the following definitions:

- Cognitive Radio [20] is a well known technology which provides flexible, efficient and reliable spectrum usage. It adapts to the present radios operating characteristics to the real-time conditions. With the help of cognitive radio technology the $S U$ s are able to intelligently detect and utilize the idle licensed frequency channels when the PUs are not present. This implements the efficient reuse of licensed channels.

- Orthogonal Frequency Division Multiplexing (OFDM) [21] is a compatible method for CR systems. OFDM enables the $S U$ s to fill the spectral gaps left by the PUs. FFT components at the OFDM systems receiver can also be used for the $S U$ s to execute the channel detection [14]. [21] proposed that the SUs' band covers multiple PUs' licensed spectrum, then the $S U_{\text {s }}$ modulate zero on the subcarriers which belongs to the detected PUs' licensed spectrum while utilizing other subcarriers for transmission.

One of the typical cognitive radio systems is shown in Fig. 2.4. $P U$ system is licensed with a certain channel. As the $P U$ s do not utilize the channel all the time, the channel is not utilized properly it's full capacity.

With the help of cognitive radio technology the $S U$ s are able to intelligently detect and utilize the idle licensed frequency channels when the PUs are not present [22]. With the participation of the $S U$, the channel efficiency can be improved. Guaranteeing the controlled interference is a crucial problem in the implementation of cognitive radio systems [23].

If a spectrum opportunity exists, the $S U$ has to detect that and then begin transmission.

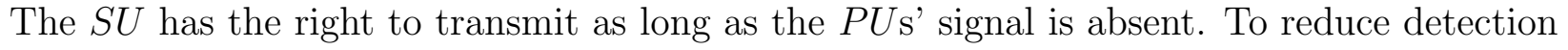
errors and collisions, a certain amount of detection probability needs to be achieved. In Fig. 2.4 active $P U_{1}$ can be detected and the interference caused by the $S U$ s' transmission to the 


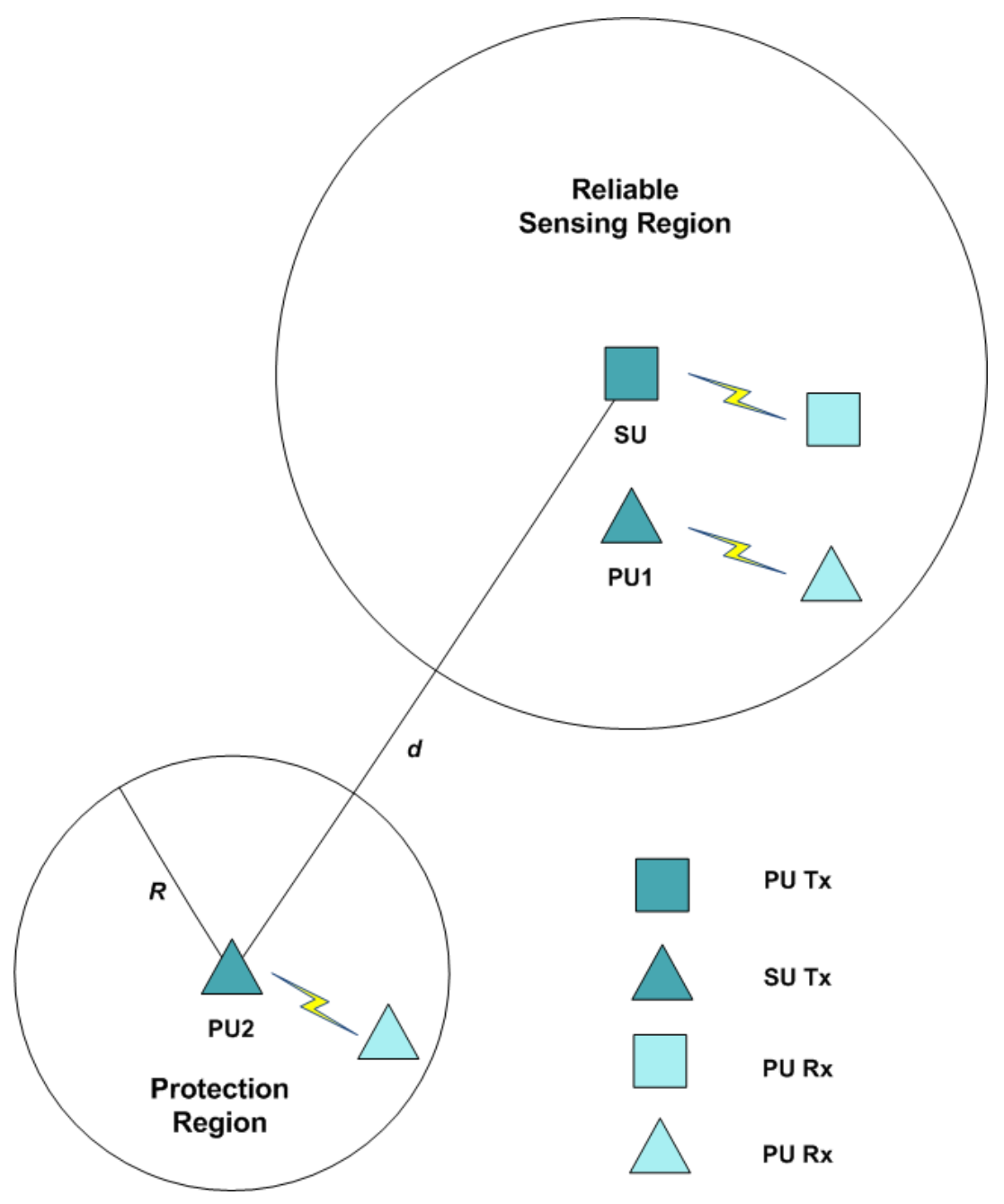

Figure 2.4: Cognitive Radio System Model

receiver of $P U_{1}$ can be circumvented. The $S U$ is unable to detect $P U_{2}$ 's signal with the required detection probability as $P U_{2}$ 's transmitter is far away from the $S U$.

In Fig. 2.4, $P U_{2}$ defines a protection area whose radius is $R$. This also requires the interference power at the margin and at a certain value, for example $\eta$. Here, $S U$ s' transmission power $P_{t x}$ is tied to a power constraint, which is given by,

$$
P_{t x} \leq \eta(d-R)^{\beta}
$$


Here, $d$ is the distance between the $S U$ transmitter and the nearest $P U$ transmitter beyond the reliable sensing region. $\beta$ is the value of the path attenuation factor. We only consider the distance based on the path loss for simplicity. In reality the constraints on the SUs' power density function is more relevant for the $P U$. To be precise just the power constraint is not enough to describe the $S U$ s' impact on the performance of the $P U$ s.

For the proper utilization of the spectrum, the opportunities left by the PUs, OFDM modulation with the ability to reconfigure subcarrier structure is a good match for cognitive radio systems as this particular system needs to be highly flexible. In OFDM-based Cognitive radio system OFDM modulation's FFT component has the ability to conduct spectrum sensing. This plays a key factor to deduct the overhead on the implementation of the cognitive radio capability.

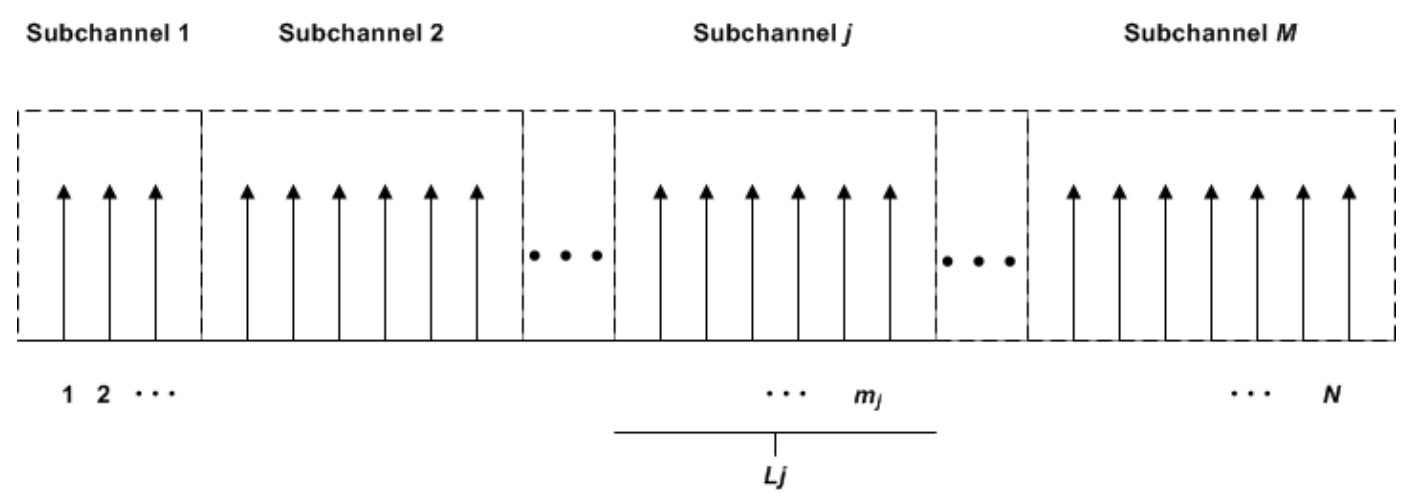

Figure 2.5: Spectrum of Secondary User in OFDM-Based Cognitive Radio Systems

For an OFDM-based cognitive radio system, as shown in Fig. 2.5, we can see that the spectrum belonging to $M$ number of $P U_{\mathrm{s}}$ can be potentially used by the $S U_{\mathrm{s}}$. We can see that the total number of subcarriers is $N$ and the $j$ th subchannel which is licensed to the $j$ th $P U$ system has $L_{j}$ subcarriers.

In this system, the $S U$ detects each subchannel before any transmission. This utilizes the proper spectrum sensing method of OFDM modulation. According to the Channel 
State Information (CSI) of the subcarriers over the $S U$ 's transmission link and the spectrum sensing results, $S U$ s can calculate their allocated power. SU's transmission can also predict different modulation types and other parameters.

The received signal of the $i$ th subcarrier within one OFDM symbol can be formulated as:

$$
y_{i}=h_{i} x_{i}+n_{i}
$$

where $x_{i}$ and $y_{i}$ are the transmitted and received signals on the $i$ th subcarrier respectively. The channel gain is represented by $h_{i} . n_{i}$ is the Normalised Additive White Gaussian Noise (AWGN) with mean 0 and variance 1.

When a $P U$ transmitter is detected to be absent in a subchannel, all the subcarriers in this particular subchannel gets modulated by zero during the transmission period, i.e., the total added power of the subcarriers in this subchannel is initially zero. Or else this subchannel can be used by the $S U$ but with a particular power constraint. This power constraint will be discussed in the next section. After channel detection we assume $G_{j}$ is the power constraint on the $j$ th subchannel, then the equation can be shown as

$$
G_{j} \triangleq \begin{cases}0 & P U_{j} \text { is detected } \\ \eta_{j}\left(d_{j}-R_{j}\right)^{\beta_{j}} & P U_{j} \text { is not detected }\end{cases}
$$

From the equation, $\eta_{j}$ is the value of the interference power constraint of $P U_{j}$ near the marginal area where the radius is $R_{j}, d_{j}$ is the distance between the $S U$ s' transmitter and the nearest undetectable $P U_{j}$ s transmitter and $\beta_{j}$ represents the path attenuation factor.

In traditional OFDM systems, optimal power allocation that maximizes the channel capacity is water-filling on the subcarriers with the total power constraint [24]. In order to avoid unacceptable interference to the $P U$ s that can not be detected due to the large distance from the $S U$, the $S U$ should limit it's transmit power even when no $P U$ is detected [25]. 
OFDM-based Cognitive Radio System introduces per subchannel power constraints. WaterFilling method is considered to be a solution to distribute power to it's maximize capacity to the $S U$ s.

\subsubsection{Iterative Partitioned Water-Filling Method}

In [14] the authors investigated the optimal power allocation strategy that aims at maximizing the capacity of OFDM-based Cognitive Radio systems. For this paper the objective function and the constraints are as follows:

$$
\begin{array}{ll}
\max _{\left\{P_{i}\right\}_{i=1}^{N}} & \sum_{i=1}^{N} \log \left(1+h_{i} P_{i}\right) \\
\text { subject to: } & P_{i} \geq 0, \forall i \\
& \sum_{i=1}^{N} P_{i} \leq P_{t} \\
& F_{j} \leq G_{j}, \forall j
\end{array}
$$

where, $N$ is the total number of subcarriers and $j=1 \ldots M$; and $M$ is the total number

of subchannels. $G_{j}$ is the power constraint on the $j$ th subchannel. $F_{j}=\sum_{i=m_{j}}^{m_{j+1}-1} P_{i}$ is the power allocated to the $j$ th subchannel and $m_{j}$ is the index of the last subcarrier in the $j$ th subchannel. A power allocation vector $\mathbf{P}$ is the solution in equations (2.21) if and only if it satisfies:

$$
P_{i}=\max \left(0, \mu_{j}-\frac{1}{h_{i}}\right)
$$

Here $i=1,2, \ldots . . N$ and $j$ is the index of the subchannel which the $i$ th subcarrier belongs to. Assume $A \triangleq\left\{j \mid F_{j}<G_{j}\right\}, B \triangleq\left\{j \mid F_{j}=G_{j}\right\}$. If $P_{t}<\sum_{j=1}^{M} G_{j}$ we have $A \neq \emptyset$. then $\mu_{j}$ is the common water-level for each subchannel and is determined by:

for $j \epsilon A$

$$
\mu_{j}=\hat{\mu}
$$




$$
\sum_{j \in A} \sum_{i=m_{j-1}+1}^{m_{j}} \max \left(0, \hat{\mu}-\frac{1}{h_{i}}\right)=P_{t}-\sum_{j \in B} G_{j}
$$

If $B \neq \emptyset$, for $j \epsilon B$

$$
\begin{gathered}
\sum_{i=m_{j-1}+1}^{m_{j}} \max \left(0, \mu_{j}-\frac{1}{h_{i}}\right) \\
\mu_{j} \leq \hat{\mu}
\end{gathered}
$$

The subcarriers with the optimal power allocation can be divided into two sets: the set $A$, i.e., the subchannels whose allocated power is strictly smaller than the corresponding per subchannel power constraint and the set $B$, i.e., the subchannels whose allocated power is equal to the corresponding per subchannel power constraint.

For subchannels in $A$, the allocated power is the result of water-filling on all the subcarriers that belong to these subchannels with power $P_{t}-\sum_{j \in B} G_{j}$. Thus, all the subchannels in $A$ have a common water level $\hat{w}$.

For each subchannel in $B$, e.g., the $j$ th subchannel where $j \epsilon B$, the allocated power is the result of water-filling on the subcarriers that belong to the $j$ th subchannel with power $G_{j}$.

Therefore, each subchannel in $B$ has a unique water level $\mu_{j}$ and satisfies $\mu_{j} \leq \hat{\mu}$, i.e., the water level of the subchannel which has a unique water level is less or equal to the common water level.

Also, based on the sufficient property of the Iterative Partitioned Water-filling Method and the strict concavity of the objective function, there is only one power allocation vector satisfying all the conditions. 
However, as in the traditional water-filling problem, it is difficult to obtain the explicit expression of the optimal power allocation vector. An intuitive algorithm directly obtained from Iterative Partitioned Water-filling Method is the exhaustive search algorithm, which is described in brief below [23]:

1) Divide all the subchannels into two sets, $A$ and $B$. There are $2^{M}$ kinds of partitions in total.

2) Remove such partitions that $P_{t}-\sum_{j \in B} G_{j} \leq 0$ for each of the rest partitions, perform traditional waterfilling on the subcarriers that belong to the $j$ th subchannel, where $j \epsilon B$, with power $G_{j}$ respectively and obtain the resulting water level $\mu_{j}$.

3) Perform traditional water-filling on all the subcarriers that belong to the subchannels in $A$ with power $P_{t}-\sum_{j \in B} G_{j}$ and obtain the common water level $\hat{\mu}$.

4) Verify each partition whether satisfies $F_{j}<G_{j}$ where $j \epsilon A$ and $\mu_{j} \leq \hat{\mu}$ where $j \epsilon B$. There is only one available partition and the corresponding power allocation vector is the solution.

Here the iterative partitioned water-filling algorithm is applied to power allocation in OFDM-based cognitive radio systems. This algorithm aims at maximizing the capacity with consideration of the per subchannel power constraints caused by the PUs' interference limits [23].

\subsection{Literature Survey}

In this section, we concisely discuss most recent algorithms reported in the open literature to solve the target power allocation problems. 
Due to its great flexibility in dynamically allocating unused spectrum among CR users, and the ease of analysis of the PUs' spectral activity [26], OFDM has already been recognized in the literature as a potential transmission technology for CR systems. Since both CR user and $P U$ may exist in side-by-side bands yet have different access technologies, mutual interference is the limiting factor for performance of both networks.

Specifically, in [27] the authors have shown that using OFDM modulation causes mutual interference between the $P U$ and CR users due to the non-orthogonality of the transmitted signals. The amount of interference introduced to the PUs' band by a Cognitive Radio Users subcarrier depends on the power allocated in that subcarrier as well as the spectral distance between that particular subcarrier and the PUs band. In order to exploit the time-varying nature of fading gains across the OFDM subcarriers, power loading algorithms have been proposed in the literature [28].

In [29] the authors investigate an optimal power loading algorithm for an OFDM-based cognitive radio (CR) system. The downlink transmission capacity of the Cognitive Radio User is thereby maximized, while the interference introduced to the $P U$ remains within a tolerable range. They propose two suboptimal loading algorithms that are less complex. They also study the effect of a subcarrier nullifying mechanism on the performance of the different algorithms under consideration. The performance of the optimal and suboptimal schemes is compared with the performance of the classical power loading algorithms, e.g., waterfilling and uniform power but variable rate loading schemes that are used for conventional OFDM-based systems [29].

The authors in [30] propose Cognitive radio (CR) systems to exploit the unbalanced spectrum utilization and by allowing $S U_{\mathrm{s}}$ to use the idle spectrum of licensed users or $P U_{\mathrm{s}}$ to gain a higher spectrum utilization. Here, one of the challenges is to detect the available spectrum bands of the PUs [31]. In this approach, the energy detection-based spectrum sensing method is the most common spectrum-sensing technique due to its low computational 
complexities and easy implementation [32] [33].

In the Opportunistic Spectrum Access (OSA) mode, the $S U$ s access the spectrum when the PUs do not use it concurrently [34]. Compared to the spectrum sharing mode, in which the PUs and $S U$ s can share the spectrum channel simultaneously, the OSA mode causes less interference to the $P U \mathrm{~s}[35]$.

When using OFDM in cognitive radio networks, the power allocation schemes for the spectrum resources will be very flexible and convenient [36]. However, it becomes very challenging to allocate power to individual subchannels in the OFDM-based cognitive radio networks. In traditional power allocation problems, water-filling algorithms are prevalent [37] [38]. Because additional interference constraints must be considered in cognitive radio networks, the water-filling algorithms are always performed iteratively to solve the power allocation problems [29] [39].

In paper [40] the authors present a new water-filling algorithm for power allocation in OFDM based CR systems. The conventional water-filling algorithm cannot be directly employed for power allocation in a CR system, because there are more power constraints in the cognitive radio power allocation problem than in the classic OFDM system. In this paper, a novel algorithm based on iterative water-filling is presented to overcome such limitations. However, the computational complexity in iterative water-filling is very high. Thus, the authors explore features of the water-filling algorithm and propose a low-complexity algorithm using power-increment or power-decrement water-filling processes [40].

The classical water-filling approach [41], which states that the transmitter should avoid using the subcarriers with poor channel conditions, has been considered as the optimal power allocation scheme for OFDM systems. A number of algorithms have been proposed in the literature for a single user case [42] [43]. These algorithms, more generally known as bit and power loading schemes, are practical implementation of the classical water-filling scheme. 
A comprehensive survey on bit and power allocation algorithms for single user OFDM systems was presented in [44]. In this paper the authors presents a solution to an energyefficient resource allocation problem which maximizes the CR (i.e., secondary) link capacity taking into account the availability of the subcarriers (and hence the reliability of transmission by CRs) and the limits on total interference generated to the PUs. They consider an energy-aware capacity expression by taking into account another factor called subcarrier availability. Optimizing such an expression saves valuable resources such as battery life by selectively allocating power to under utilized subcarriers [45].

In most of the previous works, most power allocation schemes for OFDM-based CR systems intend to improve the system throughput (or Spectrum Efficiency (SE)) [29], [46], [47][48]. [46] and [29] give the optimal and suboptimal power allocation for OFDM-based CR systems under the single interference power constraint.

The paper [47] gives the optimal power allocation for overlay and underlay OFDM-based CR systems under multiple interference power constraints. [49] studies the joint power and bits allocation schemes. [50] considers the power allocation for OFDM-based CR systems with imperfect spectrum sensing. [48] Studies the joint optimization of detection threshold and the power allocation.

In [51] the authors investigate power allocation algorithms for OFDM-based cognitive radio systems, where the intra-system channel state information (CSI) of the $S U$ is perfectly known. However, due to loose cooperation between the $S U$ and the $P U$, the inter-system CSI is only partially available to the $S U$ transmitter.

The authors in paper [51] explore the two types of $P U_{\mathrm{s}}$ being considered to have different capabilities. One is a dumb (Peak Interference-Power tolerable) system that can tolerate a certain amount of peak interference at each subchannel. Another is a more sophisticated (Average Interference-Power tolerable) system that can tolerate the interference from the 
$S U$ as long as the average interference over all subchannels is within a certain threshold. Accordingly, we introduce an interference power outage constraint, with which the outage is maintained within a target level. The outage is here defined as the probability that peak or average interference power to the $P U$ is greater than a given threshold. With both this interference-power outage constraint along with a transmit-power constraint, we propose optimal and suboptimal algorithms to maximize the capacity of the $S U$.

In [52] the authors consider a spectrum underlay network, where an OFDM-based cognitive radio (CR) system is allowed to share the subcarriers of an OFDMA-based primary system for simultaneous transmission. Instead of using the conventional Interference Power Constraint (IPC) to protect the PUs in the primary system, a new criterion referred to as Rate Loss Constraint (RLC), in the form of an upper bound on the maximum rate loss of each $P U$ due to the CR transmission, is proposed for primary transmission protection. Assuming the channel state information (CSI) of the $P U$ link, the $C R$ link, and their mutual interference links is available to the $C R$, the optimal power allocation strategy to maximize the achievable rate of the $C R$ system is derived under RLC together with $C R$ s transmit power constraint [52].

In paper [53], the authors propose a new class of sub-optimal subcarrier power allocation algorithm that significantly reduces complexity of OFDMA-based CR systems. Two suboptimal proposals, called Pre-set Filling Range (PFR) and Maximum Filling Range (MFR) are studied. Investigations show that this new power allocating algorithm allows CR systems obtain high throughput while retaining low complexity.

The different types of work related to resource allocation to $S U$ s under dynamic spectrum sharing are particularly relevant to our context. In a dynamic spectrum access environment with multiple primary and secondary users, the problem of determining the optimal number of $S U_{\mathrm{s}}$ relative to the number of $P U_{\mathrm{s}}$, which maximizes the sum throughput (primary and secondary), is addressed in [54]. The authors demonstrate by simulations the tradeoff between sum throughput maximization and $P U$ interference minimization considering im- 
perfect sensing and different levels of interference tolerance at the primary and secondary receivers. However, the interference dynamics and effects of channel fading due to the propagation environment are not considered.

In [55], the implications of two fundamental concepts in dynamic spectrum access, namely, spectrum opportunity and interference constraints, are described in terms of communication activities of $P U \mathrm{~s}$ in the neighborhood of secondary transmitters and receivers. Also, for a dynamic spectrum access environment, the parameters required to specify the interference constraints and the parameters that affect the transmission power control of $S U$ s are identified.

In [56], the problem of optimal power control for secondary users under interference constraints for primary users is formulated as a concave minimization problem. The authors propose a branch and bound algorithm to obtain the solution. A transmit power control scheme for a secondary user (i.e., cognitive radio) is proposed in [57], which exploits the location information of the primary receiver obtained indirectly through spectrum sensing, to limit the interference caused to the primary receiver. The problem of channel and power allocation for secondary users in a cellular cognitive radio network is addressed in [58]. In particular, the authors propose a heuristic-based two-phase resource allocation scheme. In this scheme, channels and power are first allocated to the cognitive radio base stations to maximize their total coverage area while maintaining the interference constraints for $P U$ s. Then each base station allocates the channels among the cognitive radios within its cell such that the total number of cognitive radios served is maximized.

In paper [59], the problem of dynamic spectrum access with QoS guarantee (in terms of minimum required signal-to interference ratio) for $S U$ s under an interference temperature constraint for $P U \mathrm{~s}$ is discussed. The problem was formulated as a convex geometric program where the globally optimal solution could be obtained under a feasible power allocation for the $S U$ s. Also, a centralized tree-pruning algorithm for removing secondary links is proposed 
for the cases where a feasible power allocation among all the $S U$ s is not possible.

In [60], a distributed power and admission control algorithm that minimizes the total transmitted power (primary and secondary users) in a cognitive radio system is proposed. The objective of the proposed algorithm is to satisfy the target signal-to-interference-noise ratio (SINR) requirements of all users. With a similar spirit, in [61], algorithms for joint rate and power allocation as well as admission control algorithms are proposed for cognitive radios in a spectrum underlay scenario which guarantee the minimum SINR and rate constraints for $S U$ s and the interference constraints for $P U$ s. Maximum transmit power constraint and the fairness performance for the secondary users are considered.

In [62] the authors present solutions for the spectrum sharing problem in a dynamic spectrum access environment. They consider the case where only mean channel gains from $S U$ s to primary receiving points averaged over short-term fading are available while either instantaneous or mean channel gains for the links among $S U$ s are available [62].

In [63], power, time slots and subcarriers are jointly optimized by two separate optimal algorithms in three steps while keep fairness among users and maximization of total capacity. However, the existence of $P U$ has not been taken into considered.

In [64], two novel algorithms are presented for subcarrier and power allocation in OFDMAbased CR networks, which are constant power subcarrier allocation and heuristic joint subcarrier and power allocation respectively. Both of them are aiming at maximizing the sum capacity while maintaining the interference power introduced in the primary network band below a predefined threshold.

In [29], an optimal and two suboptimal power loading algorithms for an OFDM-based cognitive radio system are investigated while keeping the interference introduced to the primary user within a tolerable range only. 
In [65] the authors investigate the subcarrier and power allocation problem in CR systems, where primary and CR users co-exist in adjacent bands, while keeping the total interference introduced to the $P U$ band below a certain threshold and the total power allocated to the CR users under a constraint. First, according to the different purposes of the resource allocation, several suboptimal subcarrier allocation algorithms are investigated, which are termed as Max-Rate, Min-Interference and Fair-Rate subcarrier allocation algorithm, respectively. Further, for a given subcarrier allocation three suboptimal power allocation algorithms which have less complexity are proposed and compared to the optimal power allocation algorithm [65].

OFDM-based cognitive radio networks are examined in [46], where an optimal scheme, derived via Lagrangian formulation, is proposed to maximize the downlink capacity of the cognitive users while guaranteeing the interference introduced to the primary user being below a specified threshold.

The work in [66] extends the results of [46] to the multiuser scenarios, where discrete sum rate of secondary network is maximized constrained on the interference induced to the PUs' bands and on the total transmits power. Considering networks in which multiple secondary links coexist with multiple primary links through OFDMA-based air-interface, reference [67] utilizes the dual framework [68] to provide centralized and distributed algorithms which aim at maximizing the total sum rate of secondary networks subject to interference temperature constraints specified at $P U$ s' $^{\prime}$ receivers. All the above mentioned studies, however, only consider unicast transmission.

On the other hand, multi-casting, which is an efficient means of transmitting the same content to multiple receivers while minimizing the network resource usage [69], is certainly an attractive transmission technique for secondary networks who only have a limited access to the available spectrum. 
In [69], the authors explains a practically optimal joint subcarrier assignment and power allocation scheme to maximize the weighted sum rate of all $S U$ s of an OFDM based cognitive radio multi-cast network, while satisfying the tolerable interference level induced to the $P U$ s. 


\section{Chapter 3}

\section{Algorithms and Procedures}

Due to the rapid developments of wireless communications, spectrum scarcity is becoming a serious problem. Federal Communications Commission (FCC) documents indicate that both in the time domain and the spatial domain many licensed frequency bands are severely under utilized. This is why the spectrum management is trying to allow the licensed channels to be reused by other users that do not cause intolerable interference to the license holders [70]. The license holder of a channel is called Primary Users (PUs) and all the other users re-using $P U$ 's channel is known as Secondary Users $(S U s)$.

This Chapter focuses on the proposed algorithms which exhibits better performance. Implementations of the related algorithms are also shown.

\subsection{Problem Formulation}

We use $P_{t}$ to denote the OFDM block's total power constraint and $P_{i}$ is the allocated signal power on the $i$ th subcarrier. Hence the optimal power allocation problem can be expressed by, 


$$
\begin{array}{ll}
\max _{\left\{P_{i}\right\}_{i=1}^{N}} & \sum_{i=1}^{N} w_{i} \log \left(1+\left|h_{i}\right|^{2} P_{i}\right) \\
\text { subject to: } & 0 \leq P_{i} \leq S_{i}, \forall i \\
& \sum_{i=1}^{N} P_{i} \leq P_{t} \\
& F_{j} \leq G_{j}, \forall j
\end{array}
$$

where, $N$ is the total number of subcarriers and $j=1 \ldots M$; and $M$ is the total number

of subchannels, $F_{j}=\sum_{i=m_{j}}^{m_{j+1}-1} P_{i}$ is the power allocated to the $j$ th subchannel and $m_{j}$ is the index of the first subcarrier and $m_{j+1}-1$ is the index of the last subcarrier in the $j$ th subchannel.

We can also write the last constraint as $\sum_{i=m_{j}}^{m_{j+1}-1} P_{i} \leq G_{j}$.

\subsection{Iterative Partitioned Weighted Geometric Water- filling with Individual Peak Power Constraints (IGPP)}

The target problem is to identify the optimal power allocation strategy that aims at maximizing the capacity in OFDM based cognitive radio systems. The main purpose of this research was to modify the conventional water-filling algorithm applied in general OFDM based Cognitive Radio systems due to the per subchannel power constraints and individual peak power constraints.

Objective functions with several constraints in optimizing problems have resulted researchers applying the water-filling method to solve the complex problems. Many types of algorithms have been proposed in the past regarding the Conventional Water-Filling method. CWF has limitations of it's own. The peak power constraints and the total subchannel power constraints are not included in the constraints for CWF. These constraints can make the solutions more complex and it can be time consuming to solve the problem. In order to obtain an algorithm to simplify the process and decrease the computational time we have manip- 
ulated the conventional water-filling method. This has helped us to achieve our desired solution with a less complexity.

However, the complexity of this algorithm is far too high. The decision was taken to consider $2^{M}$ kinds of partitions and thus Iterative Partitioned Water-filling (IPW) was formulated [14]. This algorithm helped to solve the following constraint:

$$
\begin{aligned}
& F_{j} \leq G_{j}, \forall j \\
& F_{j}=\sum_{i=m_{j}}^{m_{j+1}-1} P_{i} \\
& \sum_{i=m_{j}}^{m_{j+1}-1} P_{i} \leq G_{j}
\end{aligned}
$$

Geometric Water-filling (GWF) approach is also proposed to solve the weighted radio resource allocation problems.Unlike the Conventional Water-filling (CWF) algorithm, we eliminate the step to find the water level through solving a non-linear system from the Karush-Kuhn-Tucker conditions of the target problem by GWF.

Although here we are proposing an exhaustive search algorithm to obtain the optimal power allocation vector, however the complexity of this algorithm is far too high. As a result, we develop a more efficient algorithm called IGPP which combines both IPW and GWFPP to get the optimal result.

Iterative Partitioned Weighted Geometric Water-filling with Individual Peak Power Constraints (IGPP) is a simple and elegant approach proposed to solve the weighted radio resource allocation problem with peak to peak power constraint and partitioned total subchannel power constraint. The proposed IGPP algorithm requires less computation than the CWF algorithm, under the same memory requirement and sorted parameters. Furthermore, the proposed IGPP algorithm avoids complicated derivation. 


\subsubsection{IGPP Algorithm}

The subcarriers with the optimal power allocation can be divided into two sets: the set $A$, i.e., the subchannels whose allocated power is strictly smaller than the corresponding per subchannel power constraint and the set $B$, i.e., the subchannels whose allocated power is equal to the corresponding per subchannel power constraint [14].

For subchannels in $A$, the actual power allocated to different subchannels are the result of waterfilling on all the subcarriers that belong to each subchannels. The subchannels in $A$ have a common water level $\hat{w}$.

To use IPW For each subchannel in $B$, e.g., the $j$ th subchannel where $j \epsilon B$, the allocated power is the result of water-filling on the subcarriers that belong to the $j$ th subchannel with power $G_{j}[14]$.

Therefore, each subchannel in $B$ has a unique water level $w_{j}$ and satisfies $w_{j} \leqslant \hat{w}$, i.e., the water level of the subchannel which has a unique water level is less or equal to the common water level [14]. But this does not follow the peak power constraints.

Thus IGPP is introduced. For IGPP the first step is to divide all the subchannels into two sets, say $A$ and $B$ and there are $2^{M}$ partitions in total. $E$ set is a subsequence of the sequence $\{1,2, \ldots, N\}, N$ is the total number of subcarriers, $|E|$ is the cardinality of the set $E$, so $E$ can be expressed as $\left\{i_{1}, i_{2}, \ldots, i_{|E|}\right\}$.

Here, $P_{t}$ is the total power available. The next step would be to perform GWFPP for every $j$ th subchannel. We can calculate $\left\{P_{i}\right\}$ using the equations through :

$$
\begin{gathered}
P_{i}=\left[P_{n^{*}} / w_{n^{*}}+\left(d_{n^{*}}-d_{i}\right)\right] w_{i} \quad 1 \leq i \leq n^{*} \\
P_{i}=0 \quad n^{*} \leq i \leq N,
\end{gathered}
$$




$$
\begin{gathered}
n^{*}=\max \left\{n \mid P_{t}(n)>0,1 \leq n \leq N\right\} \\
P_{n^{*}}=\frac{w_{n^{*}}}{\sum_{i=1}^{n^{*}} w_{i}} P_{t}\left(n^{*}\right)
\end{gathered}
$$

Since the finite set $E$ is getting smaller and smaller until the set $\Lambda$ is empty, Algorithm GWFPP carries out at most, $N$ loops to compute the optimal solution [15]. The next steps are to remove partitions that are represented by set $\{D\}$ which is a set of subchannels which satisfies $F_{j} \leq G_{j}$ where $j \epsilon A$. For each of the remaining partitions in the remainder set $A$, the geometric water-filling is performed on the subcarriers that belong to the $j$ th subchannel.

Algorithm IGPP carries out the loops to compute the optimal solution until the set $A$ is empty. The last step would be to verify each partition whether they satisfy $F_{j} \leq G_{j}$ where $j \epsilon B$. According to the paper [14], there is only one available partition and the corresponding power allocation vector is the solution.

Based on the property of IGPP and the strict constraints on the objective function, there can be only one power allocation vector which satisfies all the conditions. In CWF problem, it is difficult to obtain the explicit expression of the optimal power allocation vector. A derived algorithm obtained from [14] and [15] forming IGPP is one of the exhaustive search algorithm, which is described below: 


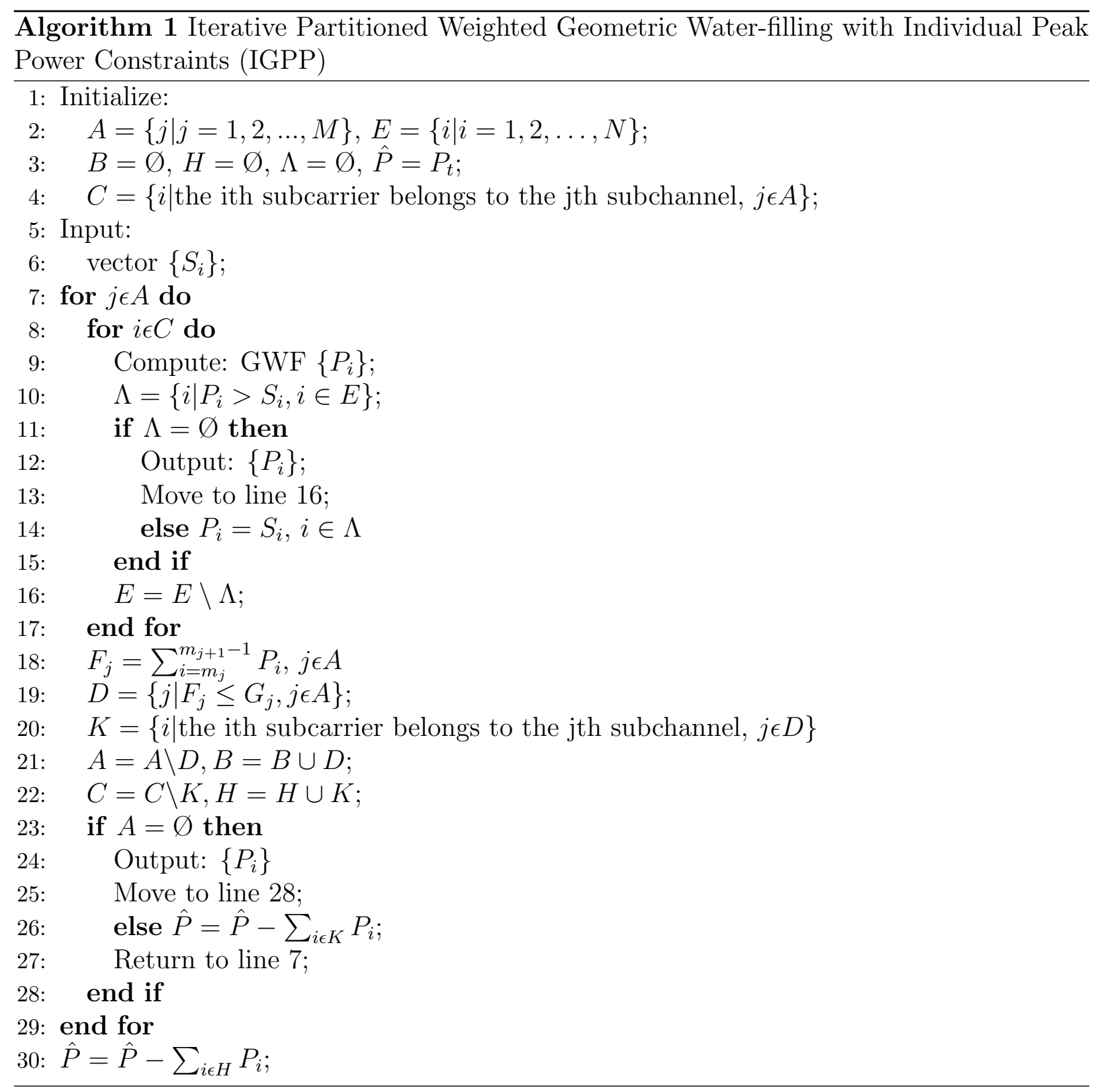




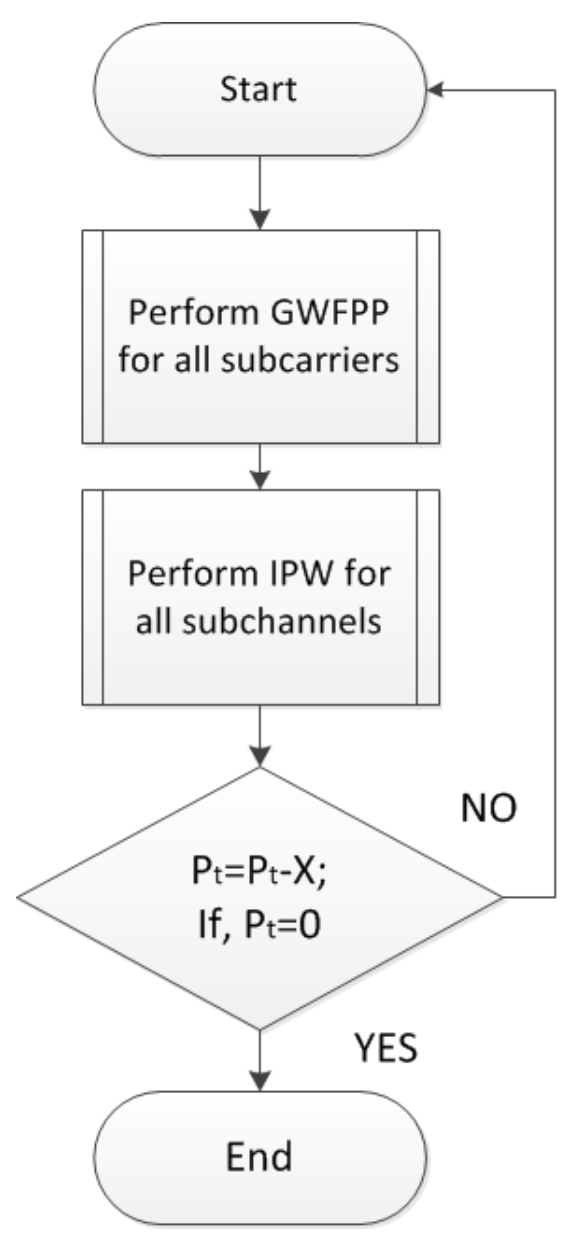

Figure 3.1: Block Diagram of IGPP

Fig. 3.1 shows the general block diagram of the pseudo code showed in the previous page. The block diagram sums up the total procedure behind IGPP. At first GWFPP is done for all the subcarriers and then IPW validates if the total subchannel power constraint is met. When both of the constraints are validated the power allocated through calculation is the $P_{i}$. This particular loop continues. As mentioned earlier $P_{t}$ is the total power and after each loop $X$ unit worth of power is deducted from $P_{t} . \quad X=\sum_{i \epsilon K} P_{i}$ which is the sum of the total power allocated through one loop. Once set $\{A\}$ becomes empty the loop exists and algorithm ends itself. Thus we obtained $\left\{P_{i}\right\}$. 


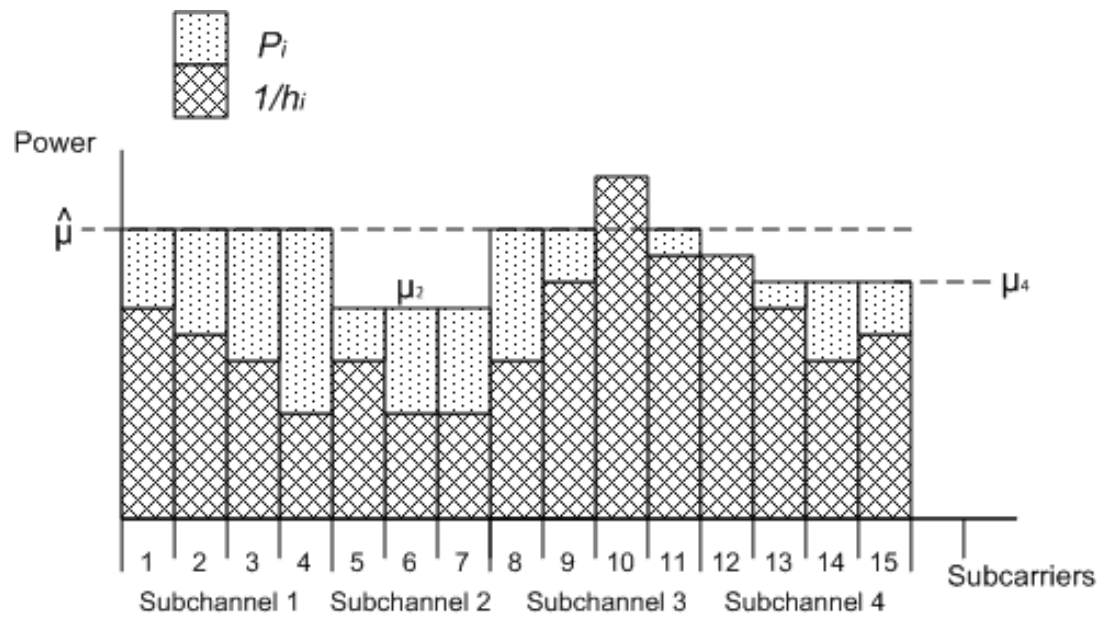

(a)

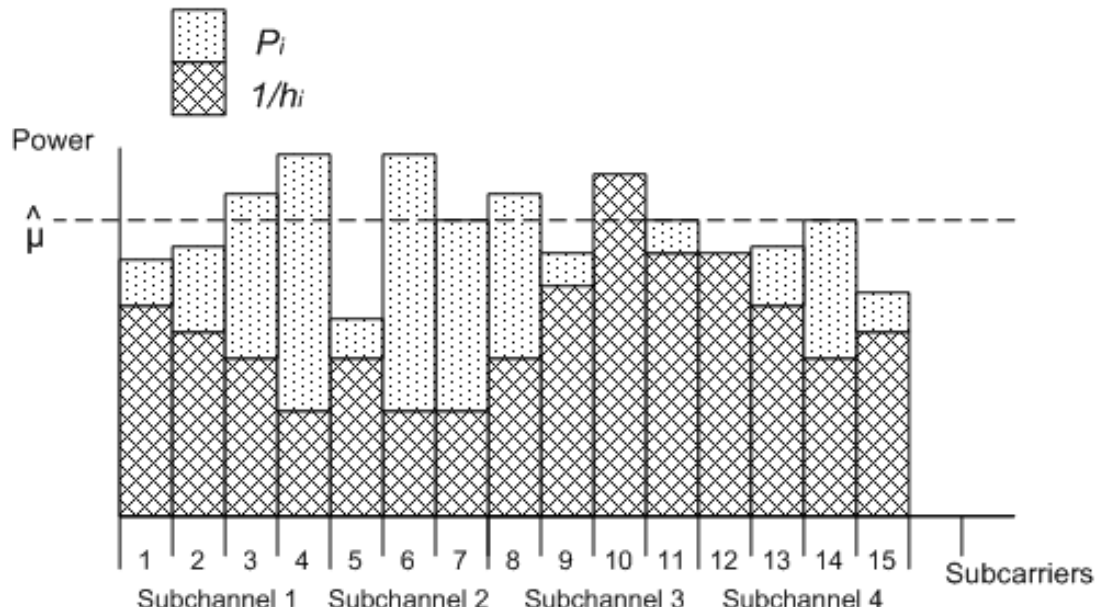

(a)

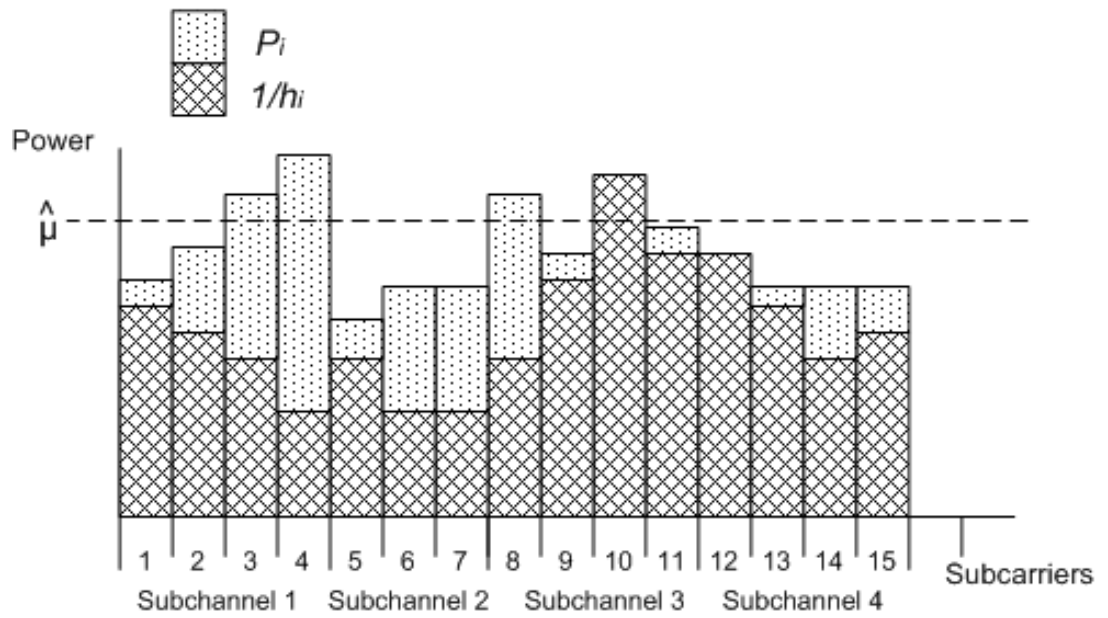

(a)

Figure 3.2: Illustration for IPW, GWFPP and the proposed Iterative Partitioned Weighted Geometric Water-filling with Individual Peak Power Constraints (IGPP). 
Fig. 3.2 illustrates the IPW, GWFPP and IGPP algorithms. Same samples of fading were selected for the three algorithms. The figure illustrates how water levels differ from one algorithm to another for the same samples of fading.

Fig. 3.2 (a) illustrates the water levels and power allocation for Iterative Partitioned Water-Filling (IPW) algorithm. The main aspect of this algorithm is to partition the sucarriers into subchannels and focus on the constraint where it dictates $F_{j} \leq G_{j}$. For CWF the water level would be the same for all the subchannels and subcarriers. In the figure $\hat{\mu}$ shows the water level for CWF approach. Where as for IPW the water level depends on the subchannels. Although the water level for the subcarriers in the same subchannels are the same but this water level might not be the same for the other subchannels. For example, Subchannel 1 and 3 have the same water level as CWF but subchannel 2 and 4 have different water level $\mu_{2}$ and $\mu_{4}$ respectively. This is due to the $F_{j} \leq G_{j}$ constraint.

Fig. 3.2 (b) illustrates the water levels and power allocation for Geometric Water-Filling for Peak Power Constraints (GWFPP) algorithm. Unlike IPW the water levels for each subcarriers are different and this is due to the peak power constraints. CWF can not fullfill $0 \leq P_{i} \leq S_{i}$ due to the fact that the water level remains the same for all the subcarriers. The figure shows the different water levels for different subcarriers.

Fig. 3.2 (c) illustrates the water level and power allocation for Iterative Partitioned Weighted Geometric Water-filling with Individual Peak Power Constraints (IGPP) algorithm. The graphical example of how IPW and GWFPP was used to create IGPP is shown in this figure. It also shows how water level and power allocation varies in each of the subcarriers and subchannels. IGPP has the ability to fullfill both of the constraints $F_{j} \leq G_{j}$ and $0 \leq P_{i} \leq S_{i}$ where as IPW and GWFPP can accommodate only one of the constraints. First each subchannels go through the GWFPP algorithm to determine the individual peak water levels and then IPW is introduced to keep the total subchannel power within the $F_{j} \leq G_{j}$ constraint. Thus optimal solution for power allocation is shown in Fig. 3.2 (c) using IGPP. 


\subsection{Dynamic Channel Sensing Iterative Method (DCSI)}

The conventional water-filling (CWF) problem has a sum power constraint under nonnegative individual powers. It can be solved by Non-Geometric Water-filling approaches. Since the solution is parameterized with a water level, the problem reduces to obtaining the water level such that the power constraint is satisfied with equality. This leads to a non-linear system, in one parameter, such as the water level, that is determined by the sum power constraint. Further, this non-linear system consists of a non-linear equation and another inequality to find the water level. In order to find the exact value of the water level, different algorithms have been proposed that can be classified into iterative algorithms and exact algorithms. The iterative algorithms are trivially implemented in practice and get close to the exact value.

For Radio Resource Allocation (RRA), one of the most typical problems is to solve power allocation using the CWF. When we consider different weight of the channels, the problem can be solved using weighted WF algorithm. As communication system develops, the structures of the system models and the corresponding RRA problems evolve to more advanced and more complicated ones like (3.1).

The CWF method cannot solve the problem stated in (3.1) of generalized problems through determining a unified water level. Thus Dynamic Channel Sensing Iterative (DCSI) algorithms is proposed to give a solution which is close to the exact value for power allocation for OFDM based Cognitive Radio Systems. 


\subsubsection{DCSI Algorithm}

In DCSI we first calculate the channel fading in the subcarriers. The subcarriers' total fading subchannels are given priority for power allocation. For example the $j$ th subchannel with total fading $\sum_{i=m_{j}}^{m_{j+1}-1} f_{i}$ which is less than the $j+1$ th subchannel with total fading $\sum_{i=m_{j+1}}^{m_{j+2}-1} f_{i}$ will be given more preference according to DCSI.

For DCSI we also consider the $G_{j}$, the total power constraint on the $j$ th subchannel. We run the algorithm based on descending $G_{j}$. This will prioritise the subchannels being more suitable for utilization. The priority will be given to the subchannel with greater $G_{j}$.

In the algorithm for ascending $i$, a loop will be generated which will automatically calculate temporary power for each subcarrier. They are denoted as $\left\{B_{M_{j}}, \ldots, B_{M_{j+1}-1}\right\}$.

After calculating $\left\{B_{M_{j}}, \ldots, B_{M_{j+1}-1}\right\}$ we run two separate loops which will determine if the temporary allocated powers abide by the actual power constraints.

We start DCSI by initializing set $\{A\}$ which represents the subchannels and set $\{E\}$ is the representation of all the subcarriers. Set $\left\{G_{j}\right\}$ is total power constraints for the $M$ number of subchannels. Set $\left\{F_{i}\right\}$ is the fading for all the subcarriers and $\left\{S_{i}\right\}$ set represents the peak power constraints for $N$ number of subcarriers. $P_{t}$ is the total given power.

The algorithmic representation of DCSI in the following page, 


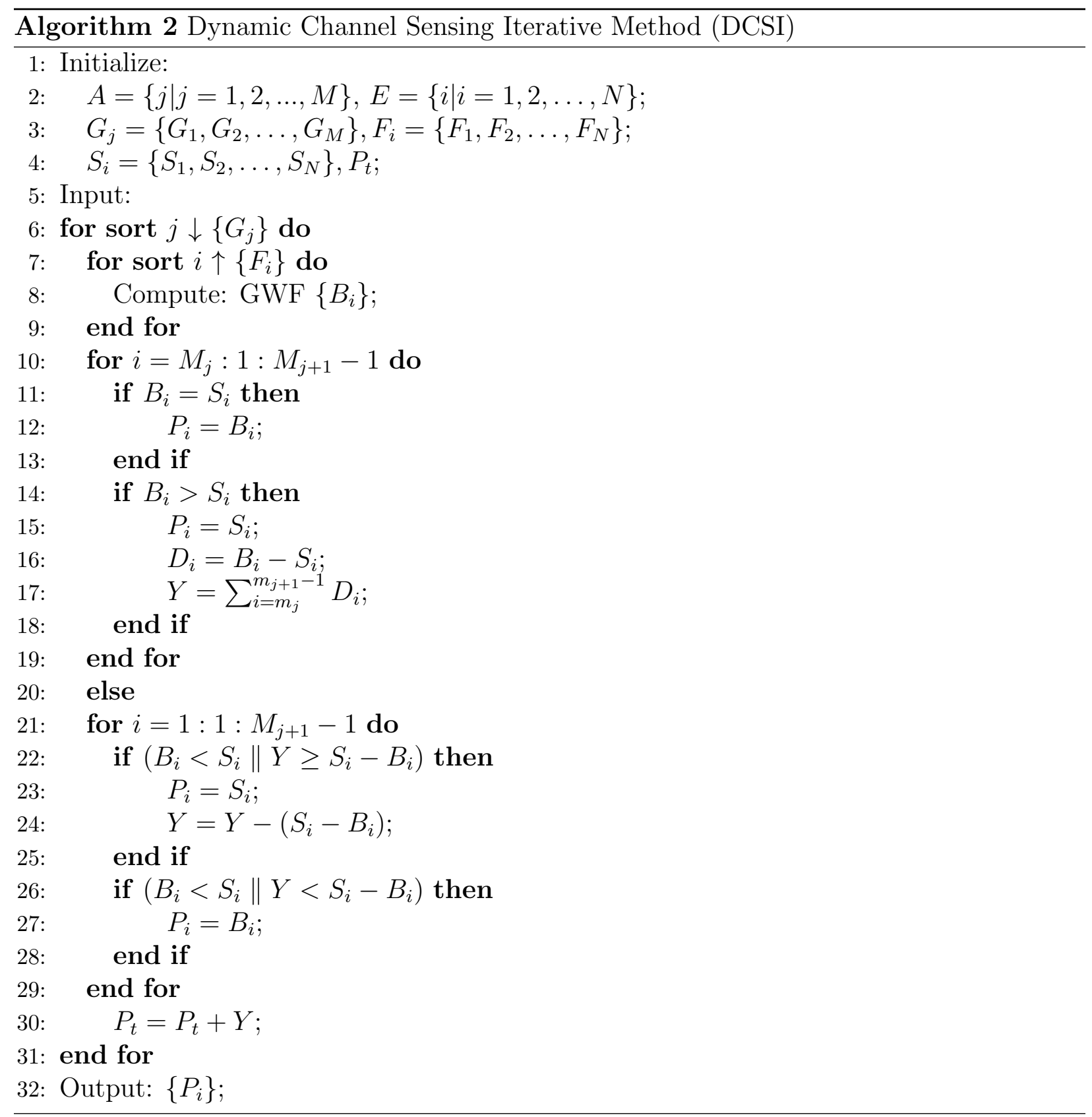




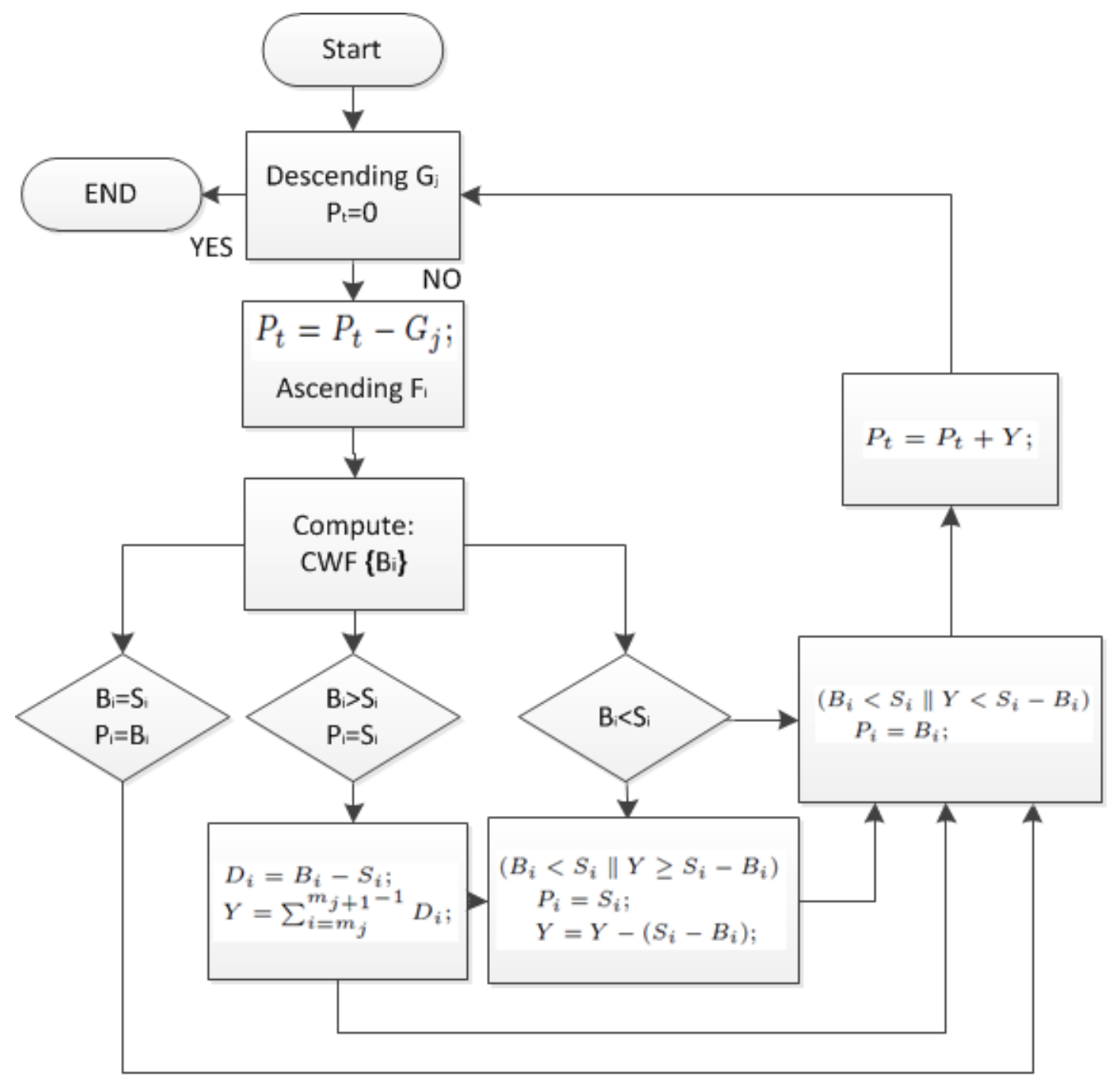

Figure 3.3: Block Diagram of DCSI

Fig. 3.3 illustrates the basic block diagram of the DCSI pseudo code captured in the previous page. $P_{t}$ is the total power and available resources and $S_{i}$ are the peak power constraints. At first the algorithm sorts the subchannels $j$ according to the descending values of $G_{j}$. This initiates the first FOR loop. Before the second FOR loop gets innitiated the algorithm provides $G_{j}$ values amount of power for the second nested FOR loop. Thus each time power is deducted $P_{t} \leq P_{t}-G_{j}$ and the updated $P_{t}$ is left for the following loops. For a particular subchannel, the algorithm then sorts the subcarriers $i$ according to the fading values of the channels which is represented by $F_{i}$. This initiates a second loop within the 
first loop. The purpose of this loop is to calculate temporary power $B_{i}$ for each subcarriers. $B_{i}$ is calculated through CWF. Once, $B_{i}$ is calculated and it is validated through three IF statements. If, $B_{i}=S_{i}$ then $P_{i}=B_{i}$, if $B_{i}>S_{i}$ then $B_{i}=S_{i}$ and the extra temporary power allocated before is deducted and we get a power value of $D_{i}=B_{i}-S_{i}$ and the accumulated value of the excess power is calculated by $Y$. If, $B_{i}<S_{i}$ the algorithm can calculate $B_{i}=S_{i}$ by compensating by the excess power $Y$. If there is any excess power, $Y$ is left over after completion of one loop we can accommodate this extra power for the next loops to come. This continues until the first loop is finished or $P_{t}$ becomes 0 .

For understanding DCSI, we use following sample values to illustrate the algorithm steps: $i=1: 11, j=1: 3, G_{1}=10, G_{2}=8, G_{3}=9, F_{1}=1, F_{2}=2, F_{3}=3$ and $F_{4}=4$.

Below is a step by step process on how to obtain the solutions,

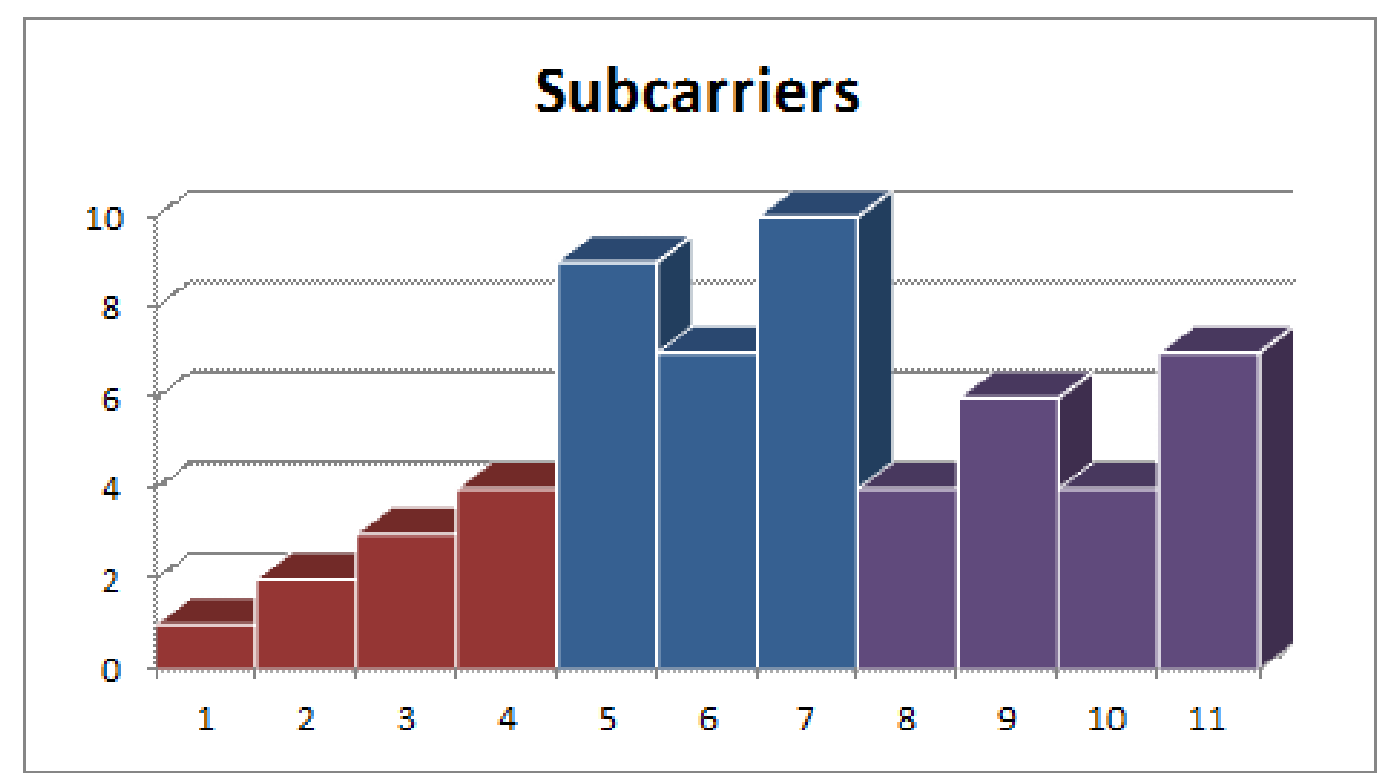

Figure 3.4: Channel Fading

In Fig. 3.4 the $\mathrm{x}$-axis represents the subcarriers and y-axis represents the fading. We first sort $\left\{G_{j}\right\}$ in an descending order. As a result $G_{j}=\left[G_{1} G_{3} G_{2}\right]$. This helps to run the 
sorted $j$ loop. The subcarriers $\{1,2,3,4\}$ are in subchannel 1 , subcarriers $\{5,6,7\}$ are in subchannel 2 and subcarriers $\{8,9,10\}$ are in subchannel 3 . We assume $P_{t}=22$.

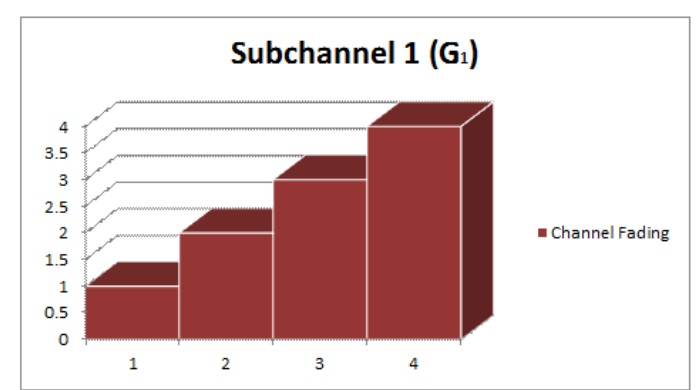

(a)

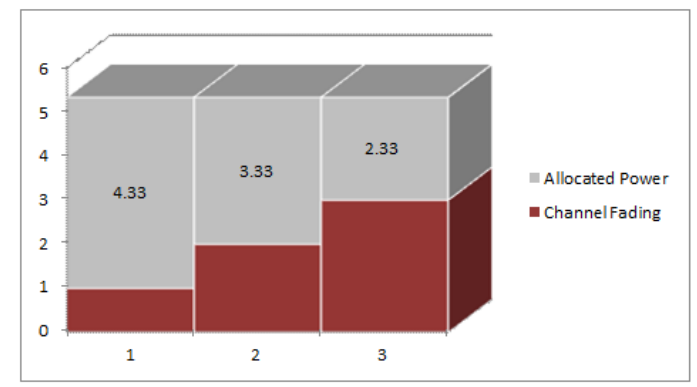

(c)

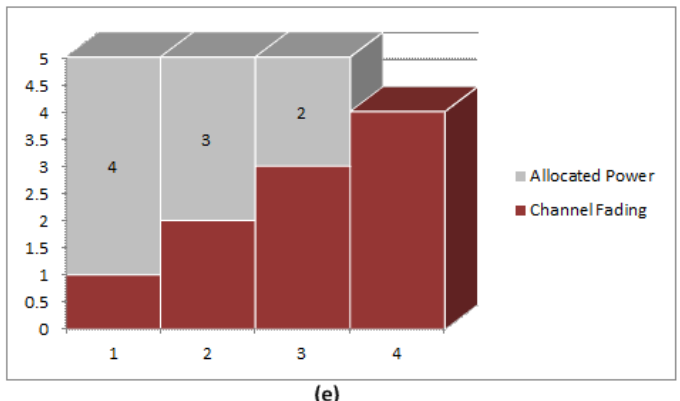

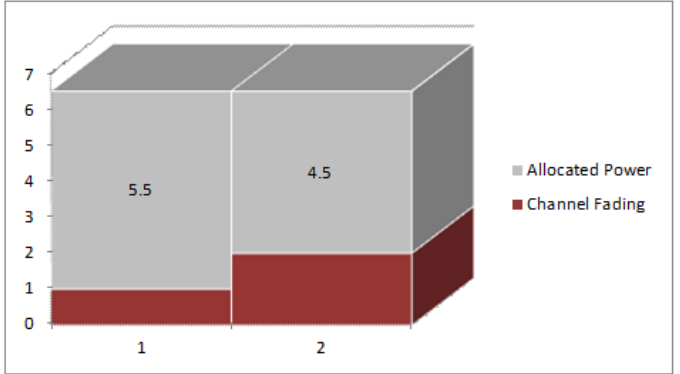

(b)

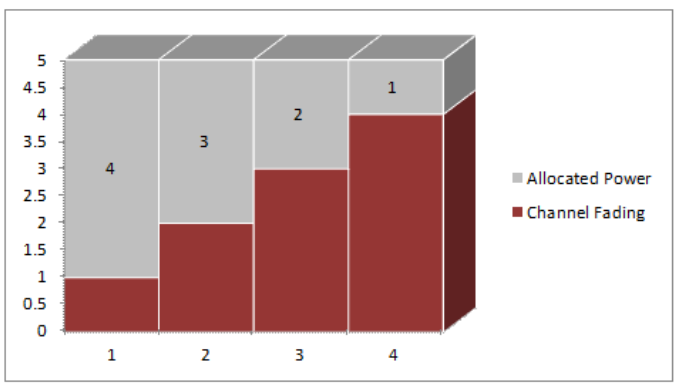

(d)

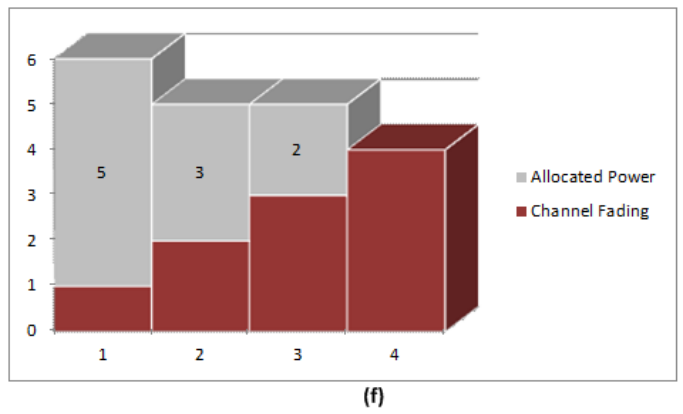

Figure 3.5: Illustration of DCSI Algorithm in Subchannel 1

In Fig. 3.5 the $\mathrm{x}$-axis represents the subcarriers and y-axis represents the fading and eventually the allocated power. From Fig. 3.5 (a) we can then sort $\left\{F_{i}\right\}$ in an ascending order. As a result $F_{i}=\left[\begin{array}{llll}F_{1} & F_{2} & F_{3} & F_{4}\end{array}\right]$ and run another loop for the sorted $i$. According to the sorted loops for the first cycle $j=1$ and $i=1$ and we calculate the temporary $P_{t}$ to be $P_{t}-G_{i}$ 
According to Fig. 3.5 (b) We can calculate $B_{i}$ by performing CWF but for visual understanding we can compute it by taking an arbitrary variable $X_{i}$ which can be calculated by, $X_{1}=\left(F_{1}+F_{2}+G_{1}\right) /(1+1)=6.5, B_{1}=X_{1}-F_{1}=6.5-1=5.5$, and $B_{2}=X_{1}-F_{2}=6.5-2=4.5$.

According to the algorithm if, $B_{i+1}+F_{i+1}>F_{i+2}$ then we continue the similar process for Fig. 3.5 (c). $X_{2}=\left(F_{1}+F_{2}+F_{3}+G_{1}\right) /(1+2)=5.33, B_{1}=X_{1+1}-F_{1}=5.33-1=4.33$, $B_{1+1}=X_{1+1}-F_{1+1}=5.33-2=3.33$ and $B_{1+2}=X_{1+1}-F_{1+2}=5.33-3=2.33$.

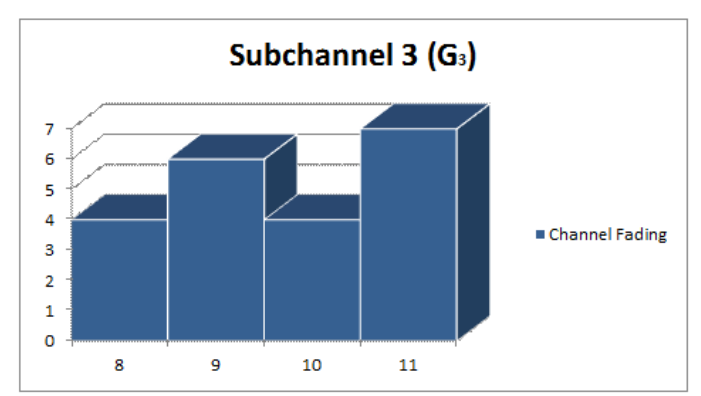

(a)

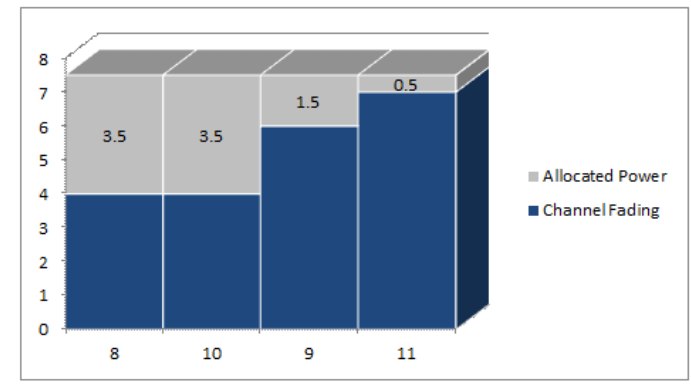

(c)

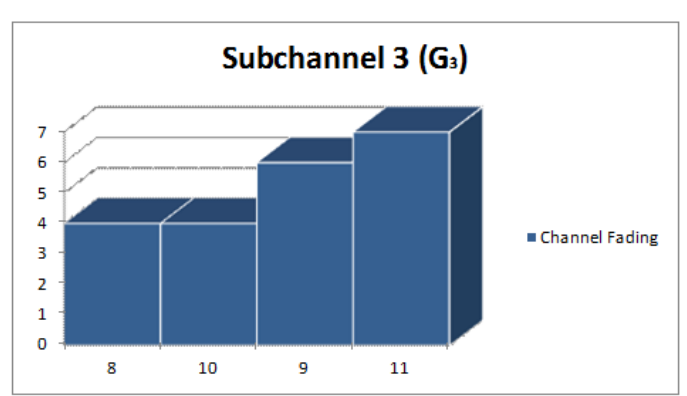

(b)

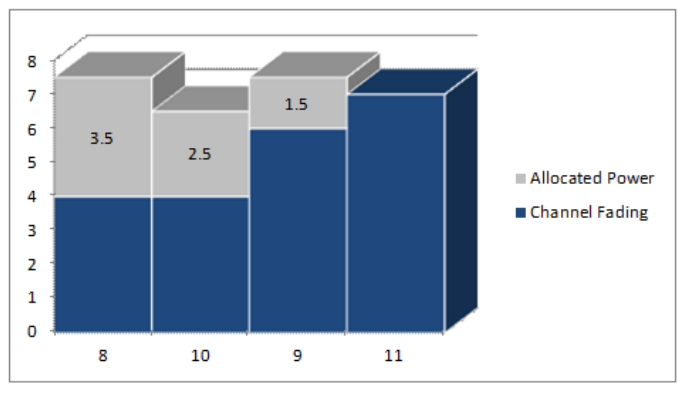

(d)

Figure 3.6: Illustration of DCSI Algorithm in Subchannel 3

According to the algorithm if, $B_{i+2}+F_{i+2}>F_{i+3}$ then we continue the similar process. According to Fig. $3.5(\mathrm{~d})$, we get $X_{3}=\left(F_{1}+F_{2}+F_{3}+F_{4}+G_{1}\right) /(1+3)=5$. We end up calculating $B_{1}=X_{1+2}-F_{1}=4, B_{1+1}=X_{1+2}-F_{1+1}=3, B_{1+2}=X_{1+2}-F_{1+2}=2$ and $B_{1+3}=X_{1+2}-F_{1+3}=1$. To check our result if they match the power constraint we run the results through two separate loops. For the first loop it checks if $B_{i}=S_{i}$ then $B_{i}=P_{i}$ and if $B_{i}>S_{i}$ then $S_{i}=P_{i}, B_{i}-S_{i}=D_{i}$ and $Y=\sum_{i=m_{j}}^{m_{j+1}-1} D_{i}$. According to these constraints 
when we go through the algorithm we get the following result illustrated in Fig. 3.5 (e). Due to peak power constraint $S_{4}=0$ no power was allocated to the 4th subcarrier as a result $P_{4}=0$.

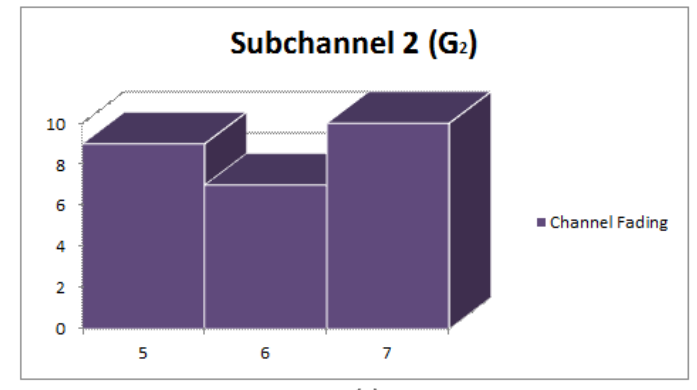

(a)

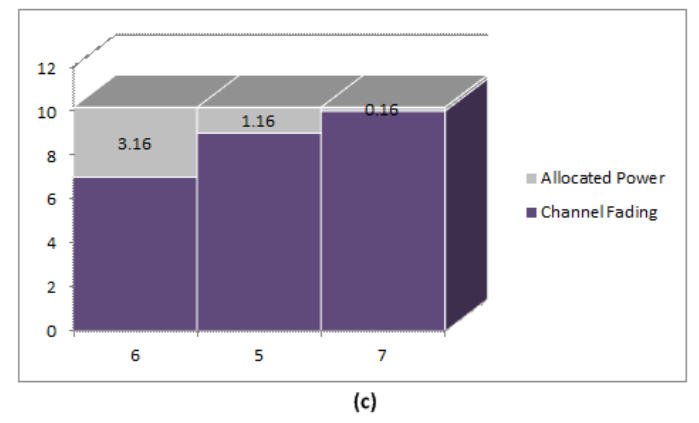

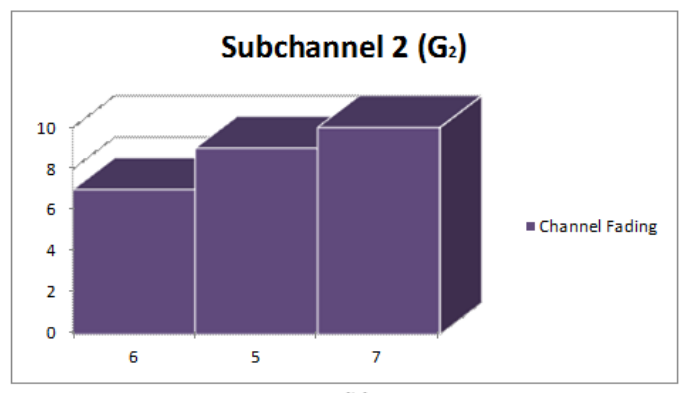

(b)

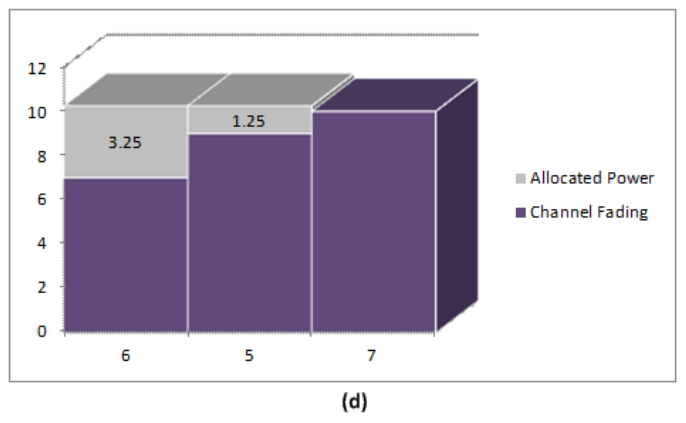

Figure 3.7: Illustration of DCSI Algorithm in Subchannel 2

The second loop calculates if $\left(B_{i}<S_{i} \| Y>S_{i}-B_{i}\right)$ then $S_{i}=P_{i}$ and $Y=Y-\left(S_{i}-B_{i}\right)$. As shown in Fig. 3.5 (f), the extra power was transferred to $P_{1}$ as $B_{1}<S_{1}$. At this point the first cycle for the sorted $i$ loop comes to an end. 


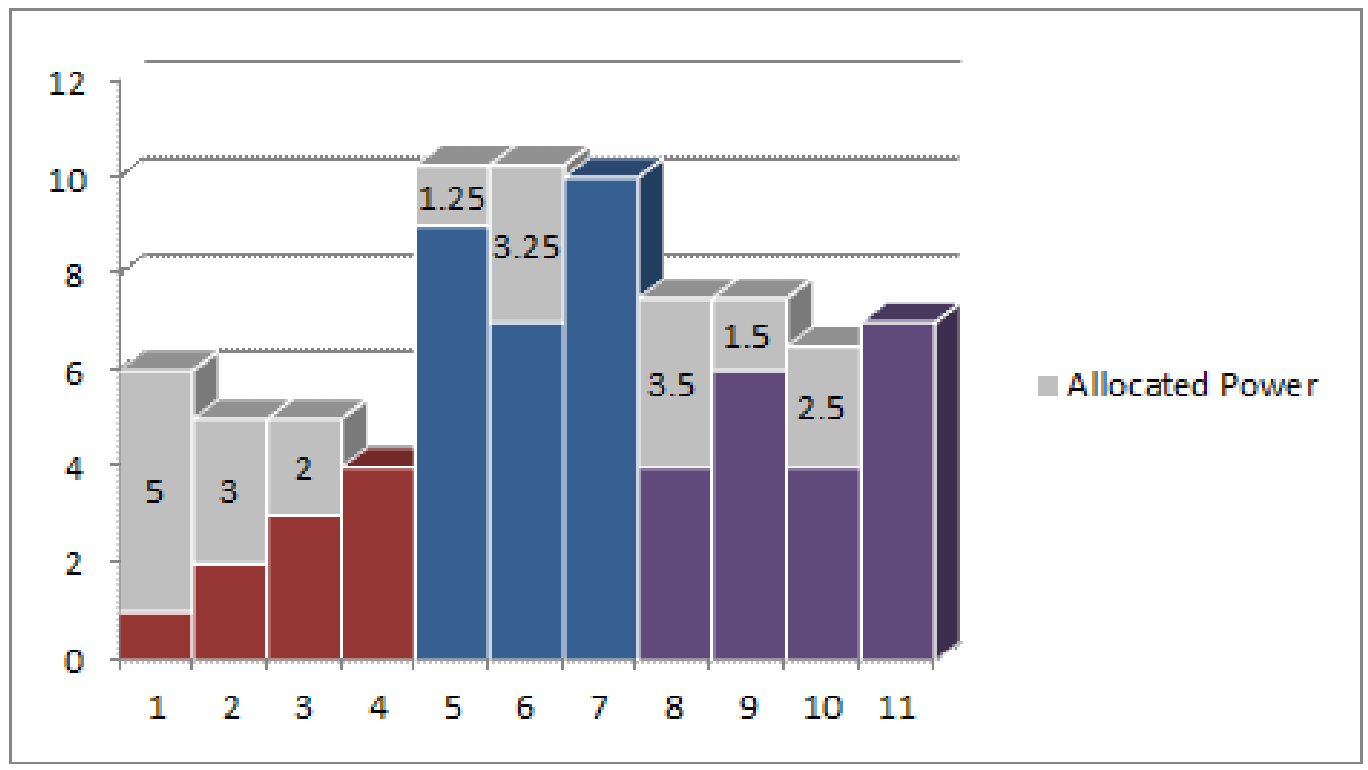

Figure 3.8: Illustration of DCSI Algorithm in all the Subcarriers

Before the loop resumes itself it must calculate the updated $P_{t}$ by $P_{t}+Y$. After the sorted $i$ loop completely finishes, the procedure for sorted $j$ loop goes through the same repetitive cycle.

The same algorithm helps to determine $P_{i}$ for the following subchannels shown in Fig. 3.6 and Fig. 3.7 where the $\mathrm{x}$-axis represents the subcarriers and $\mathrm{y}$-axis represents the fading and eventually the allocated power. After the algorithm has been fully completed we are left with set of $\left\{P_{i}\right\}$ outputs shown in Fig. 3.8. We can then calculate $\max _{\left\{P_{i}\right\}_{i=1}^{N}} \sum_{i=1}^{N} w_{i} \log (1+$ $\left.\left|h_{i}\right|^{2} P_{i}\right)$ accordingly.

DCSI, is a simple algorithm which calculates optimized $\left\{P_{i}\right\}$ through repetitive calculation. The numerical results and the comparison with Conventional water-filling method and IGPP will be given in the following chapter. 


\section{Chapter 4}

\section{Numerical Results}

In the previous chapter, the proposed Iterative Partitioned Weighted Geometric Waterfilling with Individual Peak Power Constraints (IGPP) Algorithm and Dynamic Channel Sensing Iterative (DCSI) Algorithm have been discussed. In this chapter, we show the numerical results, features, comparisons of the algorithms in details. The improvement of IGPP and DCSI over other algorithms will be illustrated in this chapter.

The proposed algorithms have been implemented in MATLAB. Randomized fading have been used to generate the outcomes of each algorithms.

\subsection{Results for Iterative Partitioned Weighted Geo- metric Water-filling with Individual Peak Power Constraints (IGPP)}

To get numerical results we generated randomised gains to illustrate the results by using IPW, GWFPP, and IGPP. For Fig. 4.2 to Fig. 4.4 we used the same set of channel gains. We assumed $P_{t}=159$.

For Fig. 4.2, IPW method was applied to get the following result. IPW is based on partition of subchannels. As a result we assumed Subcarriers $\{1,2,3,4\}$ to be in Subchannel 1, 
Subcarriers $\{5,6,7\}$ to be in Subchannel 2, Subcarriers $\{8,9,10,11\}$ to be in Subchannel 3 and the last set of Subcarriers $\{12,13,14,15\}$ to be in Subchannel 4. For each of these channels, $G_{j}=\{69,44,29,17\}$. Due to the total subchannel power constraint for the partitions, the water levels are different for each subchannel.

\begin{tabular}{|c|c|c|c|c|c|c|c|c|c|c|c|c|c|c|}
\hline Subcarriers & 1 & 2 & 3 & 4 & 5 & 6 & 7 & 8 & 9 & 10 & 11 & 12 & 13 & 14 \\
\hline & 33 & 30 & 25 & 15 & 25 & 15 & 15 & 25 & 35 & 50 & 40 & 40 & 33 & 25 \\
\hline $\begin{array}{l}\text { Peak Power } \\
\text { (Si) }\end{array}$ & 8 & 10 & 22 & 35 & 6 & 35 & 28 & 22 & 5 & 0 & 3 & 0 & 7 & 18 \\
\hline
\end{tabular}

Figure 4.1: Parameters Used For The Following Experiment

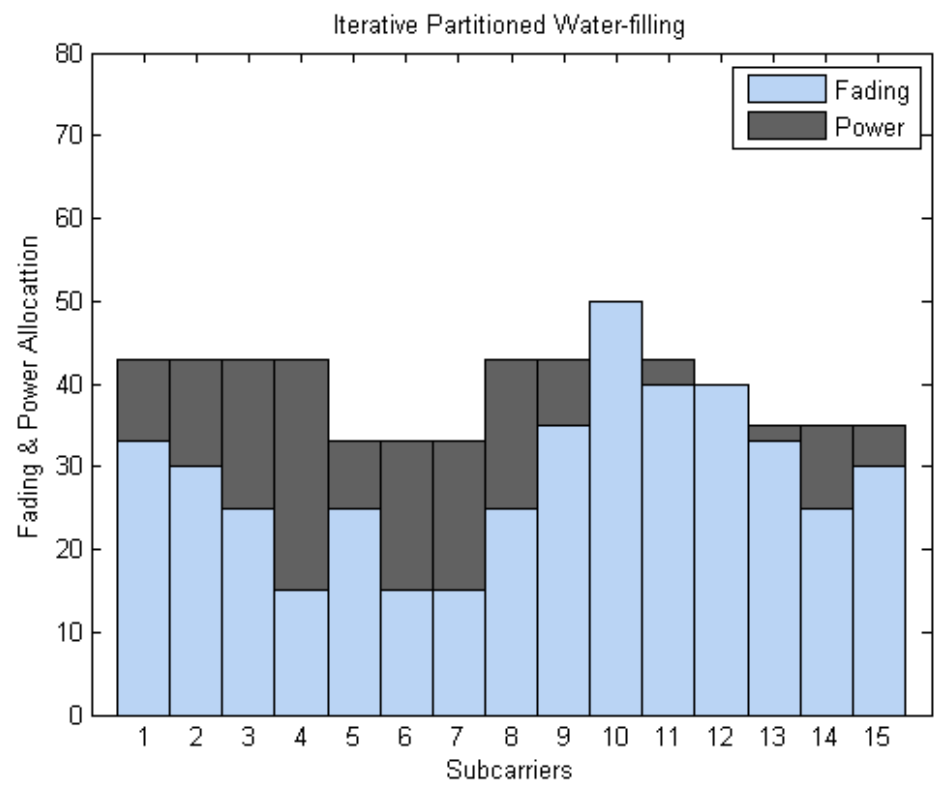

Figure 4.2: Iterative Partitioned Water-filling Method 


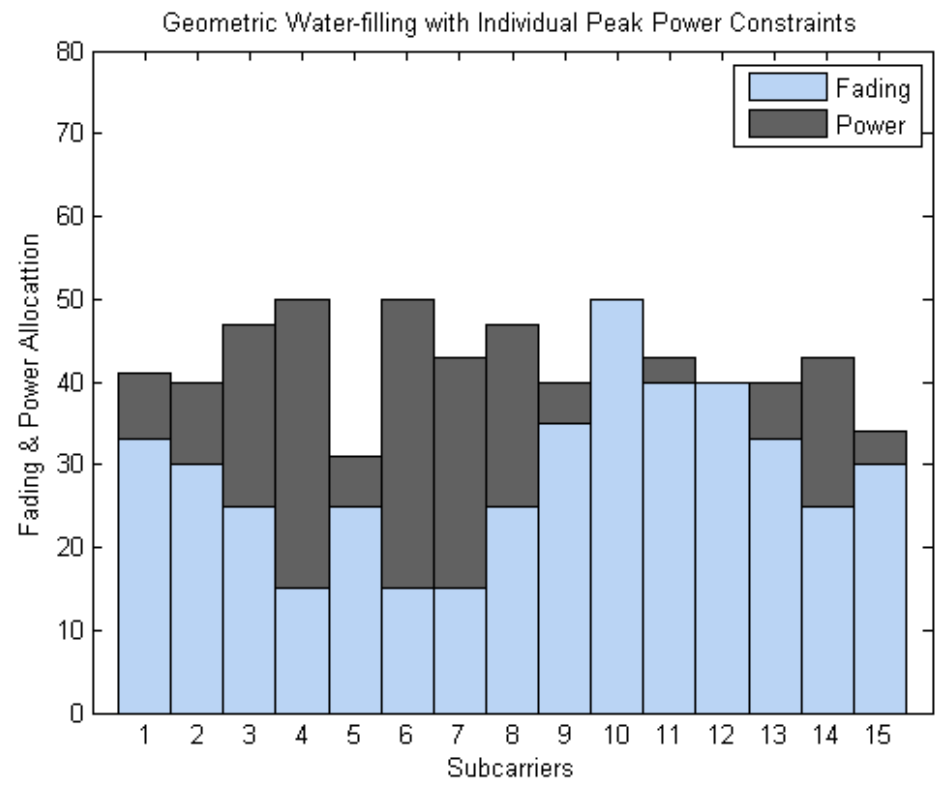

Figure 4.3: Geometric Water-filling Method with Peak Power Constraints

Fig. 4.3 is the result of GWFPP algorithm. Here the peak power constraints of our algorithm is put to test. The peak power constraint $\left\{S_{i}\right\}$ was generated by the random number function. As a result for each channel we get a different set of water level which follows the individual peak power constraints. 


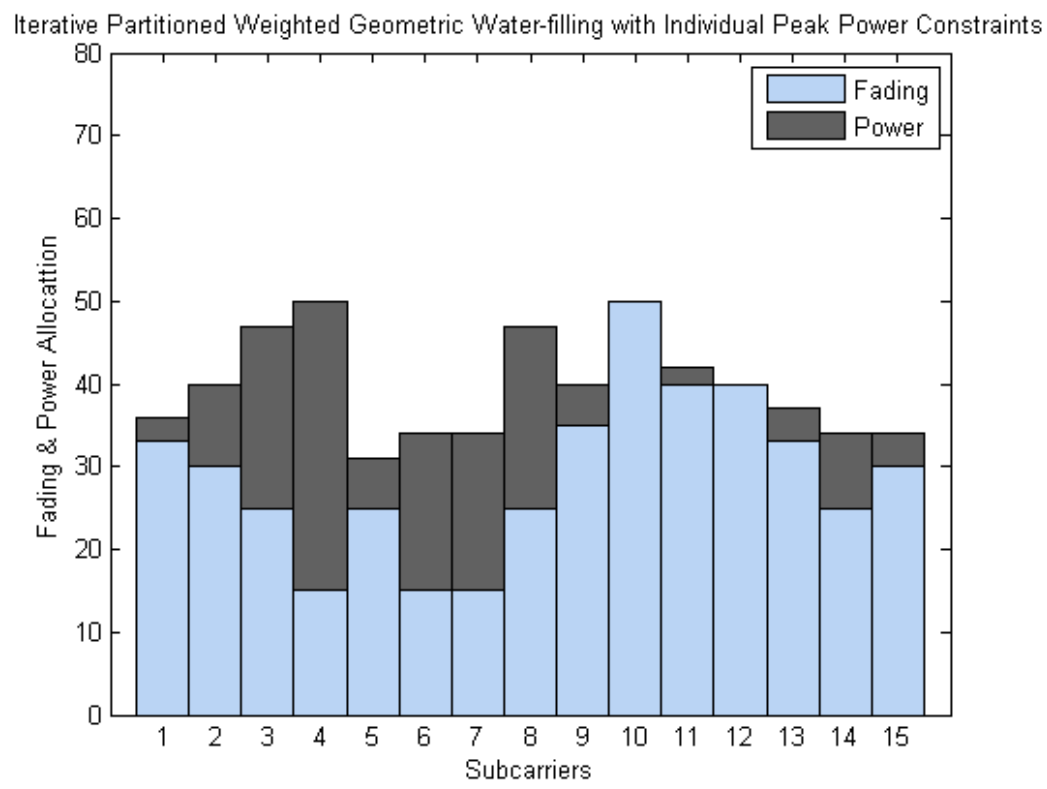

Figure 4.4: Power allocation using Iterative Partitioned Weighted Geometric Water-filling with Individual Peak Power Constraints (IGPP) Algorithm

IGPP algorithm's result is shown in Fig. 4.4. Due to the total subchannel power constraint and individual peak power constraints the numerical result varies from the results of IPW and GWFPP. For each subcarrier we get a different set of water level which follows the individual peak power constraints but they are different from the GWFPP algorithm due to the total subchannel power constraint. 


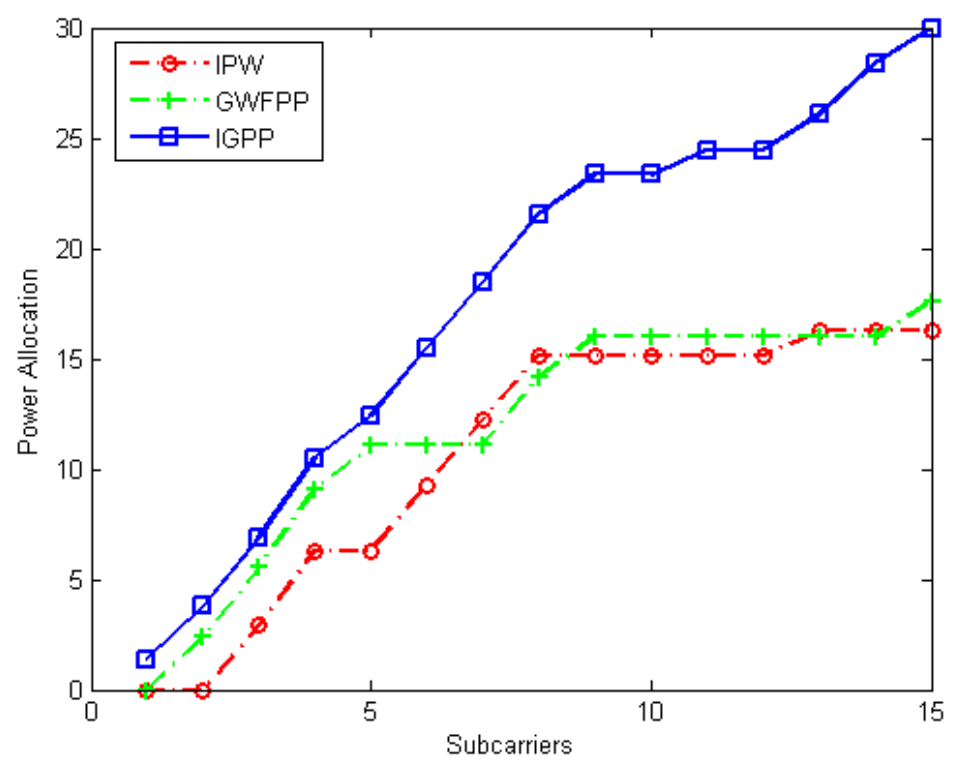

Figure 4.5: Optimal Power Allocation vs Subcarriers for IPW, GWFPP and IGPP

Fig. 4.5 compares the power allocation for the three discussed algorithms. Only the $\left\{P_{i}\right\}$ following all the constraints are shown in this figure. The IGPP graph shows that the available power resources are utilised more efficiently, where as due to the failure to meet all the constraints IPW and GWFPP algorithms' graphs fall behind. They have less power resource utilization according to the graph. Better utilisation of power resources means the throughput for that particular channel will work better. Thus optimal power allocation is important to achieve optimal throughput. IGPP allows the throughput to be better than IPW and GWFPP, due to the better utilisation of the power resources.

Table 4.1 shows the calculation of $\left\{P_{i}\right\}$ for IPW, GWFPP and IGPP. $\left\{P_{i}\right\}$ is different for each subcarrier. This table is a numerical representation of Fig. 4.2 to Fig. 4.4.

So far, we proved that the algorithm IGPP in Fig. 4.4 is a preferred solution for power allocation for OFDM based Cognitive Radio Systems. Based on our IGPP algorithm, the result of the four subchannels is illustrated in Fig. 4.4. For IGPP we can see that the water 
Table 4.1: Calculation Comparison of $\left\{P_{i}\right\}$ using IPW, GWFPP and IGPP based on the numerical results captured from Fig. 4.2 to Fig. 4.4

\begin{tabular}{|l|l|l|l|}
\hline Subcarriers & IPW & GWFPP & IGPP \\
\hline 1 & 10 & 8 & 2 \\
\hline 2 & 13 & 10 & 10 \\
\hline 3 & 18 & 22 & 22 \\
\hline 4 & 28 & 35 & 35 \\
\hline 5 & 8 & 6 & 6 \\
\hline 6 & 18 & 35 & 19 \\
\hline 7 & 18 & 28 & 19 \\
\hline 8 & 18 & 22 & 22 \\
\hline 9 & 8 & 5 & 5 \\
\hline 10 & 0 & 0 & 0 \\
\hline 11 & 3 & 3 & 2 \\
\hline 12 & 0 & 0 & 0 \\
\hline 13 & 2 & 7 & 4 \\
\hline 14 & 10 & 18 & 9 \\
\hline 15 & 5 & 4 & 4 \\
\hline
\end{tabular}

level $\hat{w}$ for each channel is different. From Fig. 4.5 and Table 4.1 we can see that the IGPP gives better result for power allocation than IPW and GWFPP algorithm.

In conclusion, the proposed Iterative Partitioned Weighted Geometric Water-filling with Individual Peak Power Constraints (IGPP) Algorithm applied to power allocation in OFDM based cognitive radio systems aims at maximizing the capacity with consideration of the per subchannel power constraints caused by the $P U_{\text {s }}$ interference limits.

\subsection{Results for Dynamic Channel Sensing Iterative Method (DCSI)}

To get the numerical results we generated randomised fading to create scenarios where IPW, GWFPP and DCSI are under test. For Fig. 4.7 to Fig. 4.9 we used the same set of 
channel fading. We assumed $P_{t}=210$.

\begin{tabular}{|c|c|c|c|c|c|c|c|c|c|c|c|c|c|c|}
\hline Subcarriers & 1 & 2 & 3 & 4 & 5 & 6 & 7 & 8 & 9 & 10 & 11 & 12 & 13 & 14 \\
\hline $1 / \mathrm{hi}$ & 40 & 30 & 50 & 20 & 30 & 40 & 30 & 30 & 20 & 30 & 20 & 70 & 40 & 50 \\
\hline $\begin{array}{l}\text { Peak Power } \\
\text { (Si) }\end{array}$ & 15 & 30 & 10 & 35 & 30 & 15 & 10 & 20 & 20 & 5 & 30 & 0 & 10 & 10 \\
\hline
\end{tabular}

Figure 4.6: Parameters Used For The Following Experiment

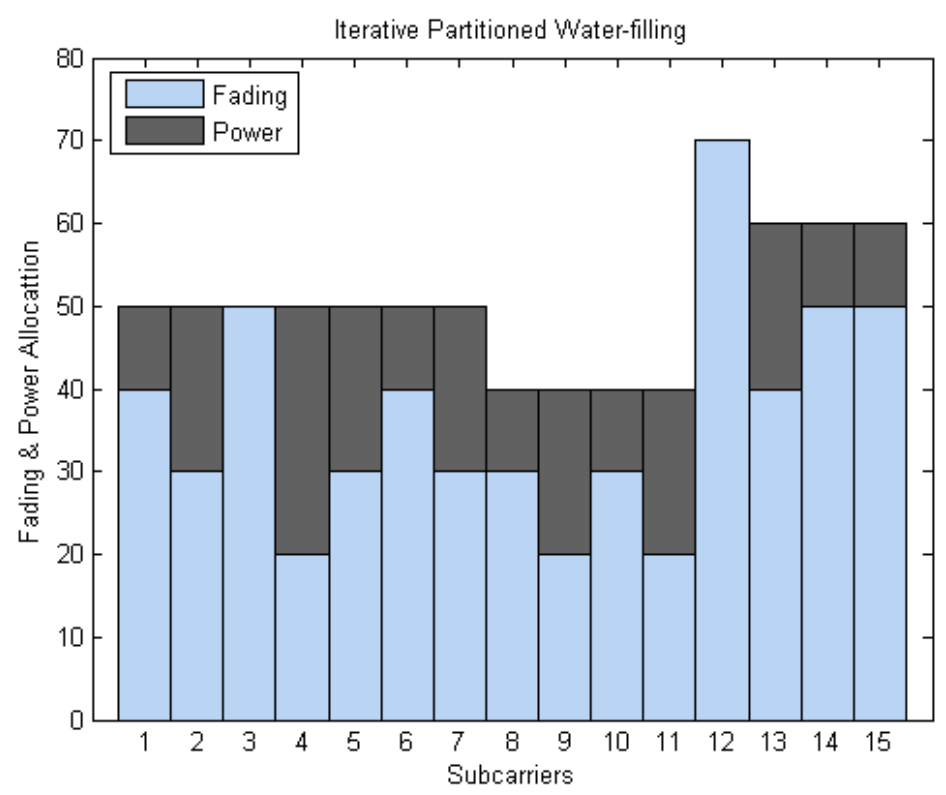

Figure 4.7: Iterative Partitioned Water-filling Method

For Fig. 4.7, IPW method was applied to get the following result. IPW is based on partition of subchannels, as a result we assumed Subcarriers $\{1,2,3,4\}$ to be in Subchannel 1, Subcarriers $\{5,6,7\}$ to be in Subchannel 2, Subcarriers $\{8,9,10,11\}$ to be in Subchannel 3 and the last set of Subcarriers $\{12,13,14,15\}$ to be in Subchannel 4. For each of these channels, $G_{j}=\{60,50,60,40\}$. From Fig. 4.7, we can see that the water level for each subchannel is different. 


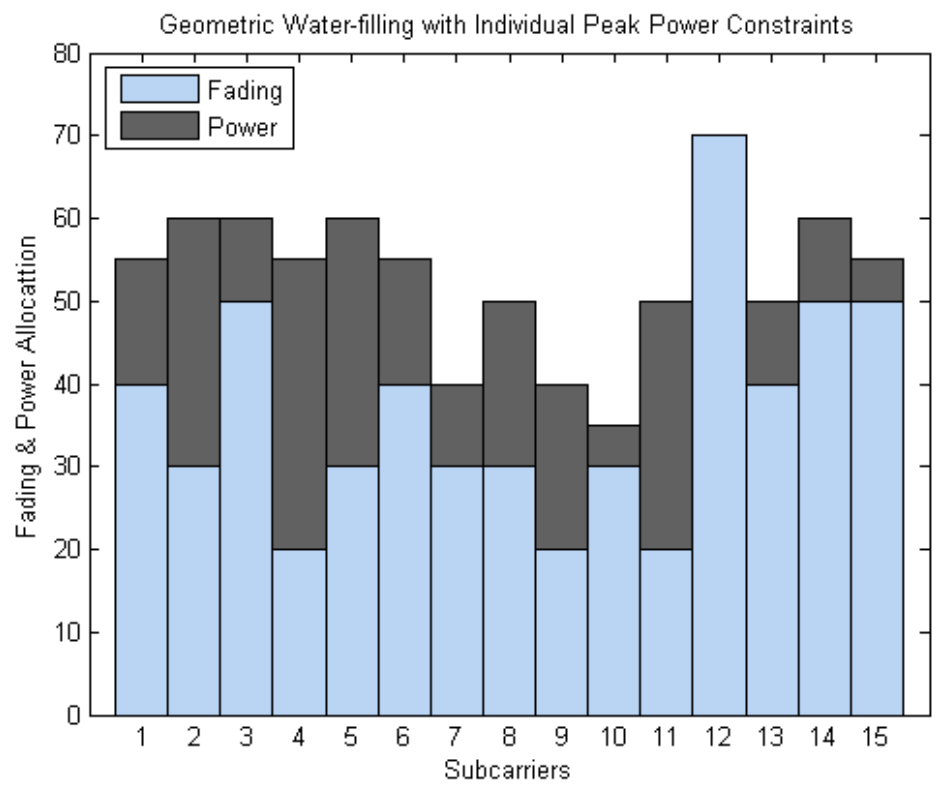

Figure 4.8: Geometric Water-filling Method with Peak Power Constraints

Fig. 4.8 is the result for GWFPP algorithm. Here the peak power constraints of our theory is put to test. The peak power constraint $\left\{S_{i}\right\}$ is generated by the random number function. As a result for each channel we get a different set of water level which follows the individual peak power constraints.

Fig. 4.9 is the result for DCSI algorithm. Due to the total subchannel power constraint and individual peak power constraints the numerical result varies from the results from IPW and GWFPP. 


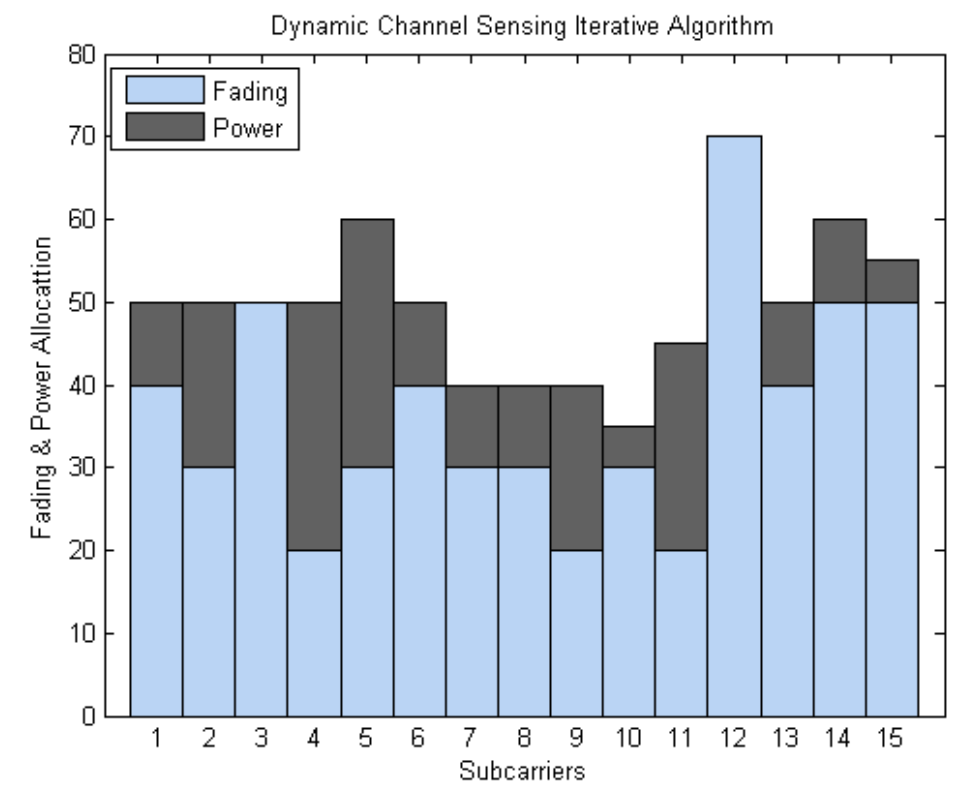

Figure 4.9: Power Allocation Using Dynamic Channel Sensing Iterative Method

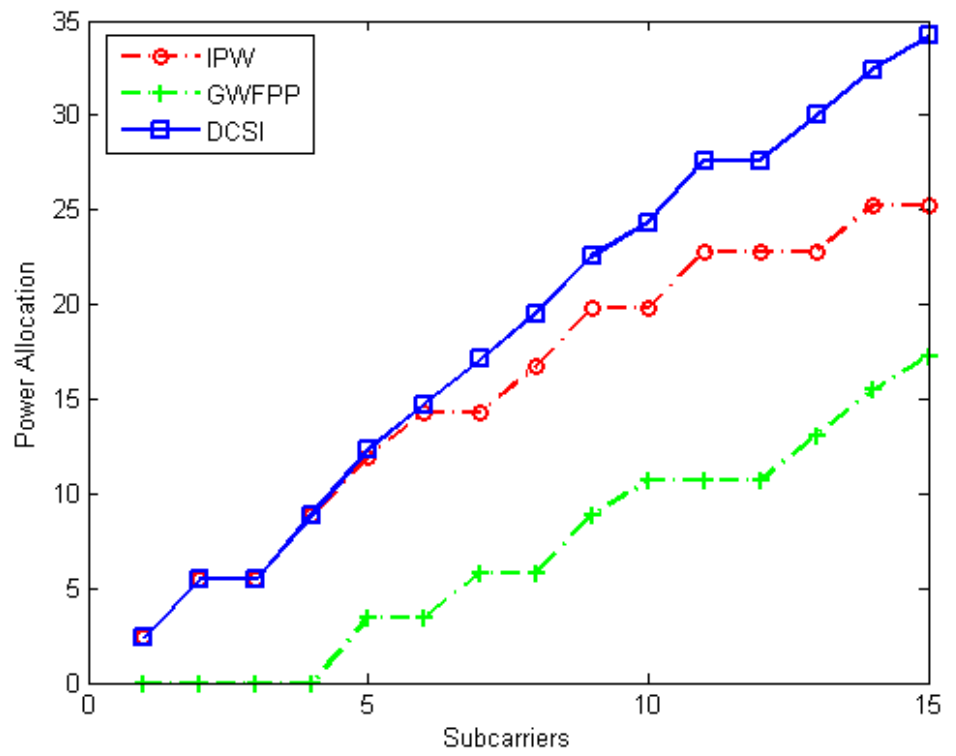

Figure 4.10: Optimal Power Allocation vs Subcarriers for IPW, GWFPP and DCSI

Fig. 4.10 shows the results for the three algorithms. Only the $\left\{P_{i}\right\}$ following all the constraints are shown in this figure. The DCSI graph shows that the available power resources 
Table 4.2: Calculation Comparison of $\left\{P_{i}\right\}$ using IPW, GWFPP and DCSI based on the numerical results captured from Fig. 4.7 to Fig. 4.9

\begin{tabular}{|l|l|l|l|}
\hline Subcarriers & IPW & GWFPP & DCSI \\
\hline 1 & 10 & 15 & 10 \\
\hline 2 & 20 & 30 & 20 \\
\hline 3 & 0 & 10 & 0 \\
\hline 4 & 30 & 35 & 30 \\
\hline 5 & 20 & 30 & 30 \\
\hline 6 & 10 & 15 & 10 \\
\hline 7 & 20 & 10 & 10 \\
\hline 8 & 10 & 20 & 10 \\
\hline 9 & 20 & 20 & 20 \\
\hline 10 & 10 & 5 & 5 \\
\hline 11 & 20 & 30 & 25 \\
\hline 12 & 0 & 0 & 0 \\
\hline 13 & 20 & 10 & 10 \\
\hline 14 & 10 & 10 & 10 \\
\hline 15 & 10 & 5 & 5 \\
\hline
\end{tabular}

was utilised properly, where as due to the failure to meet all the constraints IPW and GWFPP algorithms fall behind. They have less power resource utilization according to the graph. Better utilisation of power resources means the throughput for that particular channel would be better. Thus optimal power allocation is important to achieve optimal throughput. DCSI allows the throughput to be better than IPW and GWFPP.

Table 4.2 shows the calculation of $\left\{P_{i}\right\}$ for IPW, GWFPP and DCSI. $\left\{P_{i}\right\}$ is different for each subcarrier. This table is a numerical representation of Fig. 4.7 to Fig. 4.9.

Dynamic Channel Sensing Iterative Method (DCSI) meets every constraints of our given problem. The drawback of this algorithm is that, it has a longer computational time.

The main results of Dynamic Channel Sensing Iterative (DCSI) Algorithm in this thesis are: 
1) The Dynamic Channel Sensing Iterative (DCSI) approach can compute efficient solution to the objective function and constraints stated in this thesis, including the weighted case.

2) Machinery of the proposed DCSI approach can overcome the limitations of the CWF algorithm to include more stringent constraints.

For optimal power allocation problems with complex constraints our proposed recursive DCSI algorithm has been used to solve the problem. By iteratively applying DCSI the

computed $\left\{P_{i}\right\}$ is achieved within the boundaries of the constraints. Numerical examples from Fig. 4.7 to Fig. 4.10 are provided to illustrate the steps to obtain the optimal solutions by DSSI.

\subsection{Comparison of CWF, IGPP and DCSI}

To get numerical results we generate randomised fading to create scenarios where CWF, IPW, GWFPP, IGPP and DCSI are put to test. For Fig. 4.11 to Fig. 4.16 we use the same set of channel fading. We assumed $P_{t}=290$.

For CWF in Fig. 4.11, the water level is similar for every subchannel and their subcarriers. This does not follow any of the constraints listed in this thesis. 


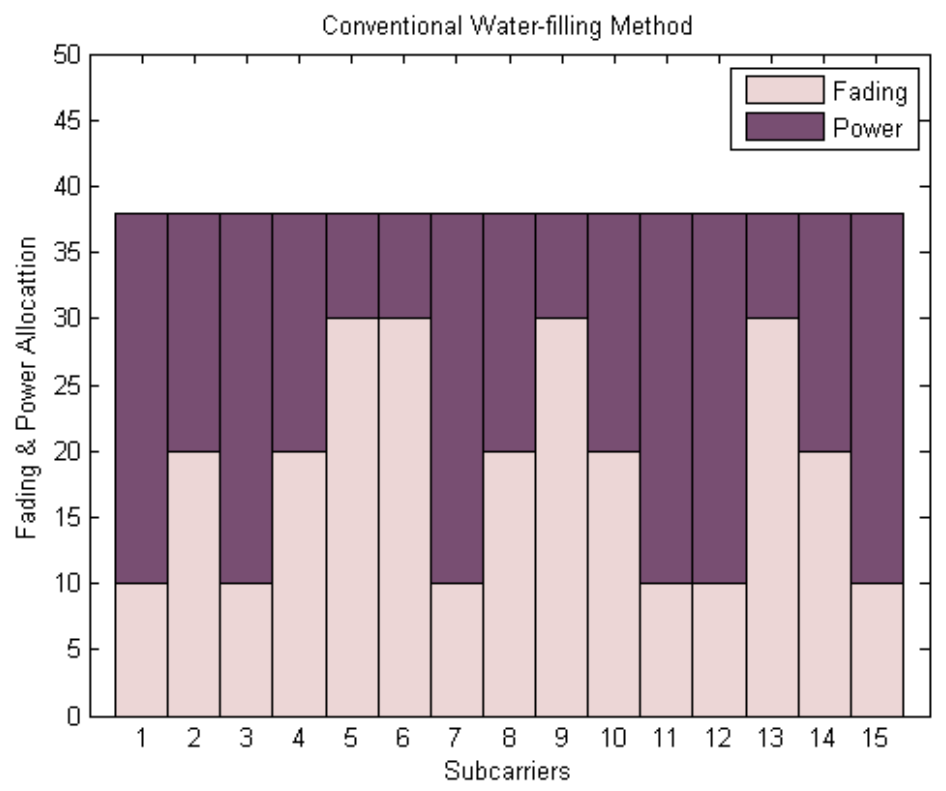

Figure 4.11: Conventional Water-filling Method

For Fig. 4.13, IPW method is applied to get the following result. IPW is based on partition of subchannels, as a result we assumed Subcarriers $\{1,2,3\}$ to be in Subchannel 1, Subcarriers $\{4,5,6\}$ to be in Subchannel 2, Subcarriers $\{7,8,9\}$ to be in Subchannel 3, Subcarriers $\{10,11,12\}$ to be in Subchannel 4 and the last set of Subcarriers $\{13,14,15\}$ to be in Subchannel 5. For each of these channels, $G_{j}=\{48,74,54,28,74\}$. We can see that the water level for each subchannel is different.

\begin{tabular}{|c|c|c|c|c|c|c|c|c|c|c|c|c|c|c|}
\hline Subcarriers & 1 & 2 & 3 & 4 & 5 & 6 & 7 & 8 & 9 & 10 & 11 & 12 & 13 & 14 \\
\hline $1 / \mathrm{hi}$ & 10 & 20 & 30 & 10 & 10 & 20 & 30 & 20 & 10 & 30 & 30 & 20 & 10 & 20 \\
\hline $\begin{array}{l}\text { Peak Power } \\
\text { (Si) }\end{array}$ & 28 & 18 & 8 & 28 & 23 & 5 & 7 & 15 & 20 & 6 & 5 & 10 & 18 & 8 \\
\hline
\end{tabular}

Figure 4.12: Parameters Used For The Following Experiment

Fig. 4.14 is the result of GWFPP algorithm. Here the peak power constraints of our theory is put to test. The peak power constraint $\left\{S_{i}\right\}$ is generated by the random number function. As a result for each channel we get a different set of water level which follows the individual peak power constraints. 


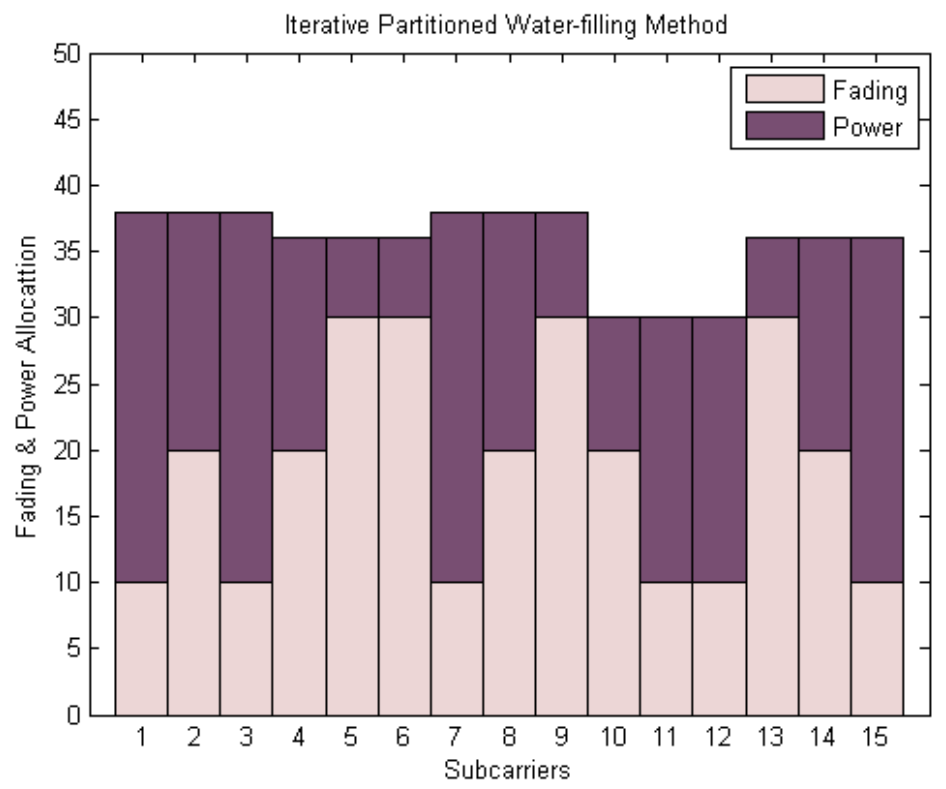

Figure 4.13: Iterative Partitioned Water-filling Method

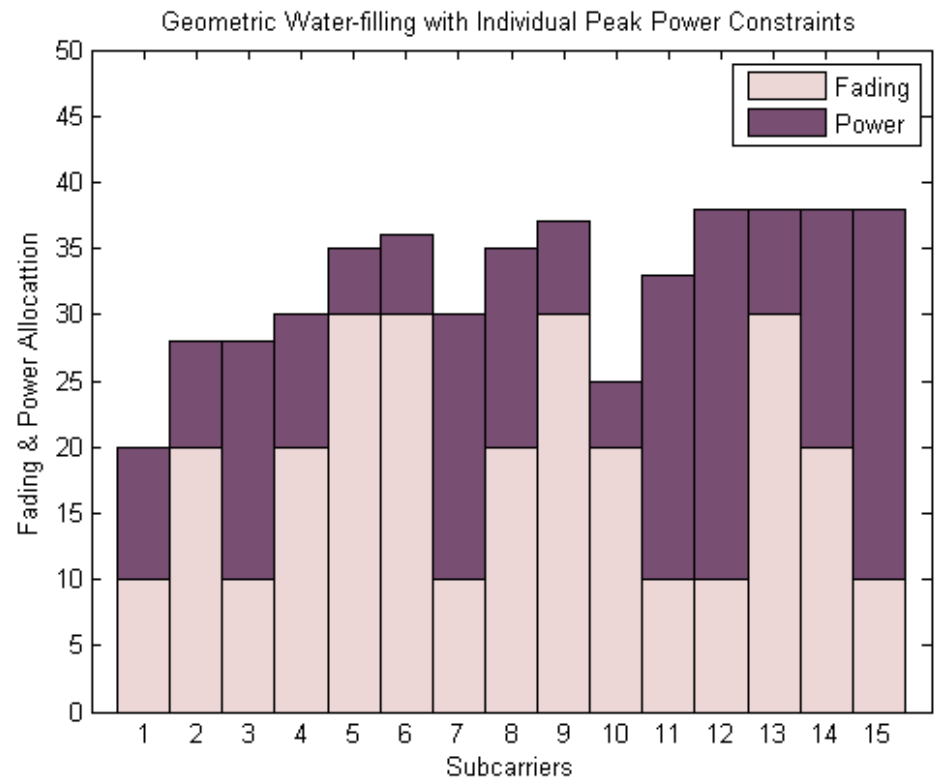

Figure 4.14: Geometric Water-filling with Individual Peak Power Constraints 
Fig. 4.15 is the result for IGPP algorithm. Due to the total subchannel power constraint and individual peak power constraints the numerical result varies from the results of IPW and GWFPP. The IGPP graph shows that the available power resources was utilised properly where as due to the failure to meet all the constraints IPW and GWFPP algorithms fall behind. They have less power resource utilization according to the graph.

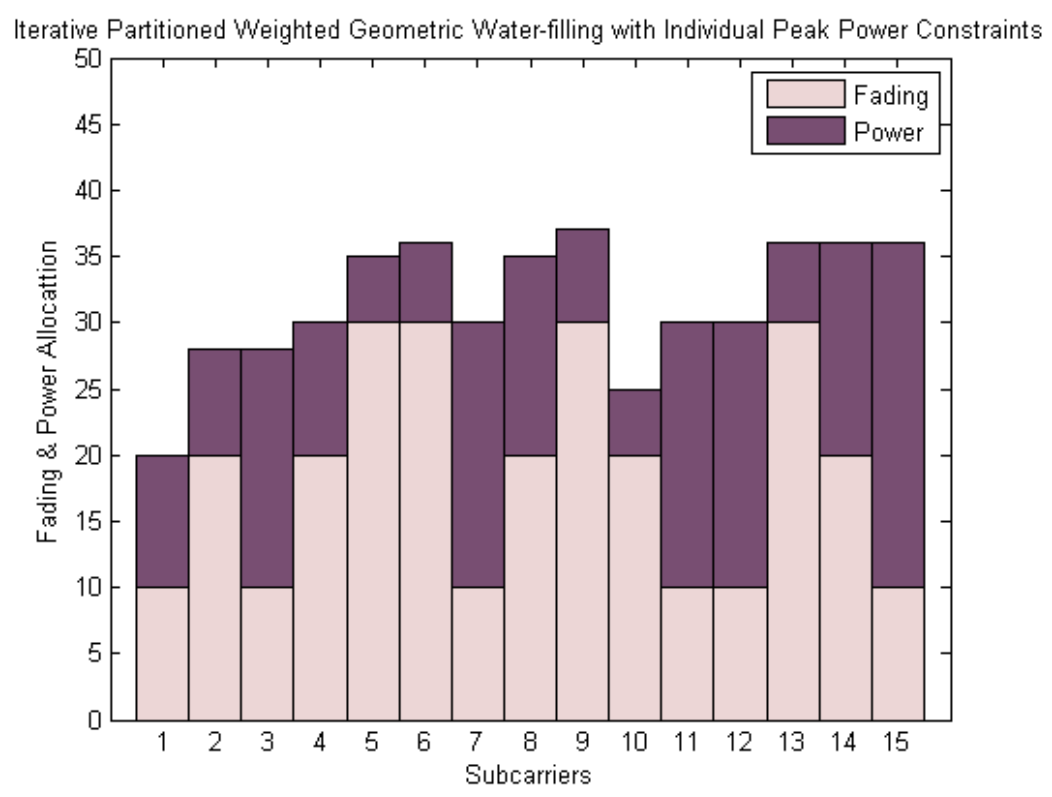

Figure 4.15: Power allocation using Iterative Partitioned Weighted Geometric Water-filling with Individual Peak Power Constraints (IGPP) Algorithm

With DCSI, all of the given constraints are crucial and play a vital role in terms of $\left\{P_{i}\right\}$. The performance is slower as the algorithm checks the constraints individually and starts from the very beginning to get the accurate result. The whole process continues in a loop until all the subcarriers in a set has proper allocated power. Fig. 4.16 is the result for DCSI algorithm. Due to the total subchannel power constraint and individual peak power constraints the numerical result varies from the results of IPW and GWFPP. 


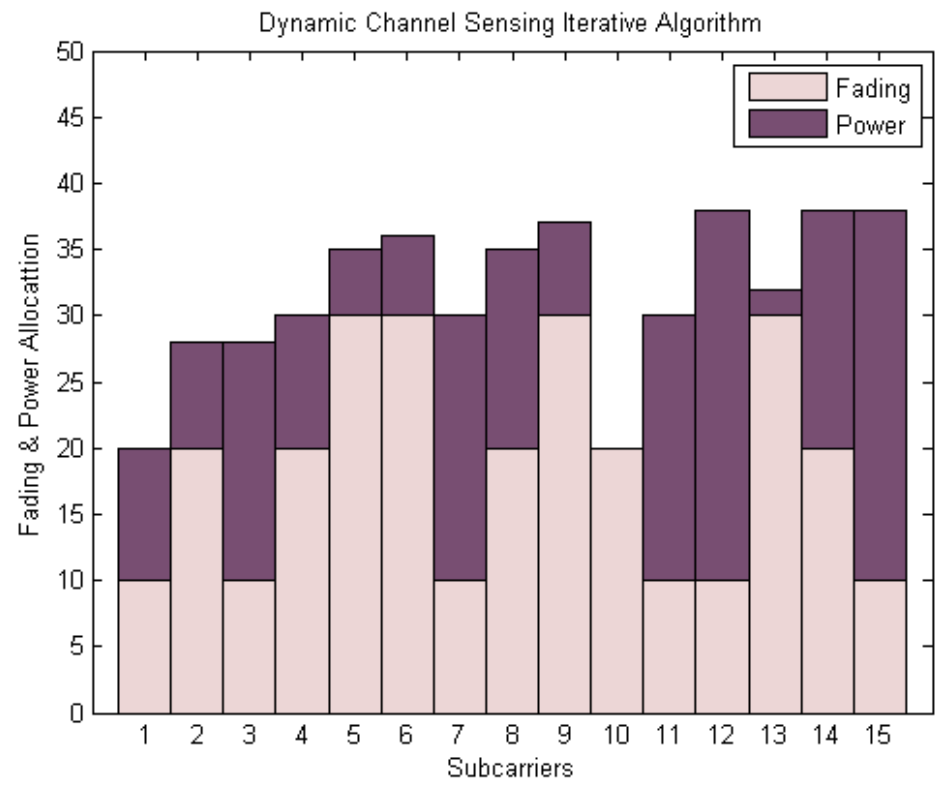

Figure 4.16: Power Allocation using Dynamic Channel Sensing Iterative (DCSI) Algorithm

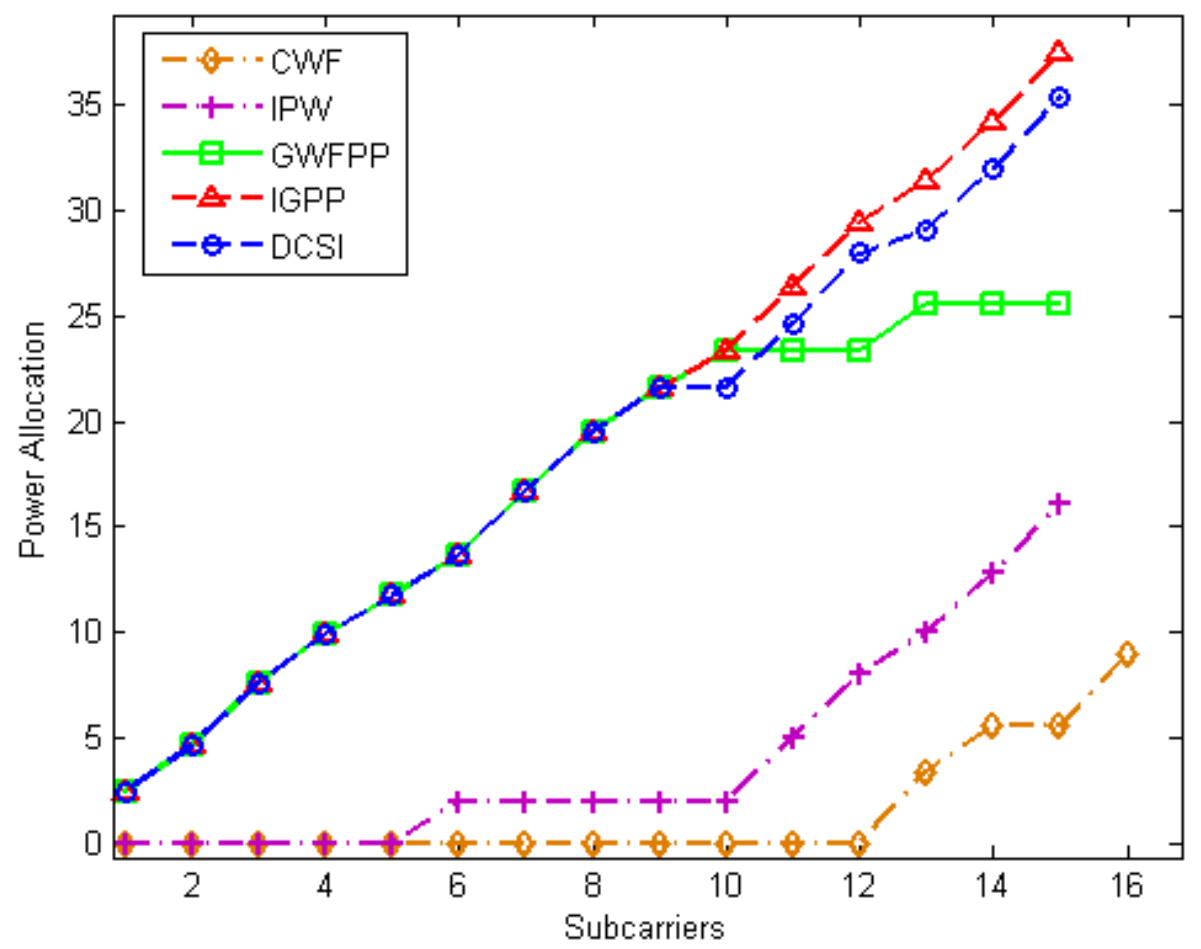

Figure 4.17: Comparison of Power Allocation with Different Algorithm. 
Fig. 4.17 is a Subcarrier Vs Power Allocation graph for all of the algorithm listed above. In this graph each algorithm has a different result. As CWF algorithm fails to meet all the constraints it has a simpler result. IGPP and DCSI are far more advanced. We used random fading per channel to get the simulated scenario. IGPP and DCSI fits all the constraints criteria and manages to simplify the problem.

After studying the five bar charts in Fig. 4.11 to Fig. 4.16 we can reach a feasible conclusion before the actual compilation of the simulated algorithms.

In Fig. 4.11 the constraints for the objective function is violated. In the simulation test for power allocation using water-filling method these subchannels are nullified due to not meeting the objective function constraints.

Fig. 4.15 illustrates, IGPP Algorithm is used to allocate power for each subcarriers. In this particular method all of the objective function constraints are met.

In Fig. 4.16 we can see a similar trend as Fig. 4.15, DCSI meets all the constraints.

There are two important aspect of DCSI algorithm which can be observed from Fig. 4.17. The first one is that, it can sense the channel and it's fading and then intelligently detects the channel with less fading and transfers extra power resources to that particular channel. Secondly, it sorts the $\left\{G_{j}\right\}$ in a descending order in order to allocate the first set of resources to the subchannel with higher total subchannel constraint. These are two important aspects which differentiate DCSI from IGPP. From numerical results of DCSI and IGPP, both algorithms are better for power allocation. 


\section{Chapter 5}

\section{Conclusion and Future Work}

As spectrum scarcity is becoming a serious problem, the worth of finding a general solution for such issue has become even serious due to the rapid development of wireless communications. Initially the main purpose of the search was to modify the conventional water-filling algorithm applied in general OFDM based Cognitive Radio systems due to the per subchannel power constraints and individual peak power constraints.

For Radio Resource Allocation (RRA), one of the most typical problems is to solve power allocation using the Conventional Water-filling. As communication system develops, the structures of the system models and the corresponding RRA problems evolve to more advanced and more complicated ones. Conventional Water-filling (CWF) is not enough to approach these sort of problems.

In this thesis, we investigated towards identifying the optimal power allocation strategy that aims at maximizing the capacity in OFDM based Cognitive Radio Systems. The main

purpose of the research was to modify the CWF algorithm applied in general OFDM based Cognitive Radio systems according to the per subchannel power constraints and individual peak power constraints.

In order to obtain an algorithm to simplify the process and decrease the computational time, we manipulated the Conventional Water-filling (CWF) method to achieve two of our 
desired solutions with a less complexity and minimum computational time.

Iterative Partitioned Weighted Geometric Water-filling with Individual Peak Power Constraints (IGPP) is the first algorithm proposed in this thesis. It is a simple and elegant approach proposed to solve the weighted radio resource allocation problem with peak to peak power constraint and partitioned total subchannel power constraint . The proposed IGPP algorithm requires less computation than the CWF algorithm, under the same memory requirement and sorted parameters.

The second iterative algorithm proposed in this thesis, similar to water-filling algorithm which abides by all the constraints is called Dynamic Channel Sensing Iterative (DCSI) Algorithm.

The proposed approaches solved the given objective function and the constraints with less derivation and computational time.

IGPP is an algorithm which is a combination of Geometric Water-filling method with Peak Power Constraint [15] and Iterative Partitioned Water-filling method described in paper [14].

DCSI is an innovative approach which allows us to solve the target problem intelligently with less complexity. It provides straightforward power allocation analysis, solutions and insights with reduced computation over other approaches under the same memory requirement and sorted parameters.

Numerical examples are provided to illustrate the effectiveness of the algorithms. Our results show with the complicated structured problem conventional water-filling method is limited due to the fact that the water levels are no longer unique. Thus IGPP and DCSI algorithms are appropriate for equivalent complex scenarios. 
As the next step, we plan to continue working on the proposed algorithms to solve more complex problems. By analyzing the accuracy and simulation time of the proposed method to innovate a general formula, we will enhance the usability of this method. More-over, in next upcoming parts we will expand our algorithm to accelerate other steps to achieve more complex objective functions with more complex constraints. 


\section{Bibliography}

[1] P. K. Garg, T. R. Dua, and A. Chandra, "Spectrum challenges for modern mobile services," ICT Standardization, vol. 1, no. 2, pp. 137-158, 2013.

[2] T. Yucek and H. Arslan, "A survey of spectrum sensing algorithms for cognitive radio applications," IEEE Communications Surveys and Tutorials, vol. 11, no. 1, pp. 116-130, 2009.

[3] A. M. Wyglinski, M. Nekovee, and Y. T. Hou, "Cognitive radio communications and networks principles and practice," Information Theoretical Limits on Cognitive Radio Networks, pp. 1-41, 2010.

[4] L. Litwin, "An introduction to multicarrier modulation," Potentials, IEEE, vol. 19, no. 2, pp. 36-38, 2001.

[5] V. Sridhar and R. Prasad, "Nothing wrong with spectrum sharing," 2014. http://www.thehindubusinessline.com/opinion/nothing-wrong-with-spectrumsharing/article 4992619.ece.

[6] E. Britannica, "Radio spectrum," 2014. http://kids.britannica.com/comptons/art164539/Commercially-exploited-bands-of-the-radio-frequency-spectrum.

[7] F. Akyildiz, W. Y. Lee, M. C. Vuran, and S. Mohanty, "Next generation dynamic spectrum access cognitive radio wireless networks: A survey," Computer Networks Journal, vol. 50, no. 13, pp. 2127-2159. 
[8] S. Haykin, "Cognitive radio: Brain-empowered wireless communications," 2005. http://bul.ece.ubc.ca/SimonHaykinCognitive.

[9] "Notice of proposed rule making and order: Facilitating opportunities for flexible, efficient, and reliable spectrum use employing cognitive radio technologies," Federal Communications Commission, vol. 11, no. 3, p. 108, 2005.

[10] "United states frequency allocation chart," Federal Communications Commission, 2003.

[11] S. K. Jones and T. W. Philips, "Plan for tests of prototype personal/portable tv white space devices," Technical report, FCC, 2008.

[12] J. Mitola, "Cognitive radio for flexible mobile multimedia communications," IEEE Mobile Multimedia Conference, 1999.

[13] I. Poole, "Ofdm orthogonal frequency division multiplexing tutorial," 2014. http://www.radio-electronics.com/info/rf-technology-design/ofdm/ofdm-basicstutorial.php.

[14] P.Wang, M.Zhao, L.Xiao, S.Zhou, and J.Wang, "Power Allocation in OFDM-Based Cognitive Radio Systems," Global Telecommunications, GLOBECOM. IEEE, pp. 4061 $-4065,2007$.

[15] P.He, L.Zhao, S.Zhou, and Z.Niu, "Water-Filling: A Geometric Approach and it's Application to Solve Generalized Radio Resource Allocation Problems," Wireless Communications, IEEE Transactions, vol. 12, no. 7, pp. 3637 - 3647, 2013.

[16] D. P. Palomar and J. R. Fonollosa, "Practical algorithms for a family of waterfilling solutions," IEEE Transaction on Signal Processing, vol. 53, no. 2, pp. 686-695, 2005.

[17] Q.Qi, A.Minturn, and Y.Yang, "An Efficient Water-Filling Algorithm for Power Allocation in OFDM-Based Cognitive Radio Systems," Systems and Informatics (ICSAI), International Conference., pp. 2069 - 2073, 2012. 
[18] R. G. Gallager, "Information theory and reliable communication," New York: Wiley, 1968. http://link.springer.com/book/10.1007/978-3-7091-2945-6.

[19] T. M. Cover and J. A. Thomas, "Elements of information theory," New York: Wiley, 1991. ftp://ftp.esat.kuleuven.ac.be/pub/stadius/referenties/CoverThomas/0.pdf.

[20] S.Haykin, "Cognitive Radio: Brain-Empowered Wireless Communications," IEEE Journal on Selected Areas in Communication., vol. 23, no. 2, pp. 201-220, February 2005.

[21] T.Weiss and F.Jondral, "Spectrum Pooling: An Innovative Strategy for the Enhancement of Spectrum Efficiency," IEEE Communications Magazine., vol. 42, no. 3, pp. S8S14, March 2004.

[22] S.Yan, P.Ren, and F.Lv, "Power Allocation Algorithms for OFDM-Based Cognitive Radio Systems," Wireless Communication Networking and Mobile Computing, pp. 1-4, 2010.

[23] P.Wang, "A Genral Power Allocation Algorithm for OFDM-Based Cognitive Radio Systems," IEEE International Conference on Communications (ICC), pp. 1-5, June 2009.

[24] D.Tse and P.Viswanath, "Fundamentals of Wireless Communication," Cambridge University Press, May 2005. http://www.eecs.berkeley.edu/ dtse/taiwancourse.pdf.

[25] Q.Zhao and B.M.Sadler, "A Survey of Dynamic Spectrum Access: Signal Processing, Netwroking and Regulatory Policy," IEEE Signal Processing Magazine., vol. 55, no. 5, pp. 2294-2309, May 2007.

[26] T. Weiss and F. K. Jondral, "Spectrum pooling: an innovative strategy for the enhancement of spectrum efficiency," IEEE Communication Magazine, vol. 43, no. 3, pp. 8-14, 2004.

[27] T. Yucek and H. Arslan, "Mutual interference in ofdm-based spectrum pooling systems," IEEE Vehicular Technology Conference, vol. 4, pp. 1873-1877, 2004. 
[28] T. Keller and L. Hanzo, "Multicarrier modulation: a convenient framework for timefrequency processing in wireless communications," Proc. IEE, vol. 88, no. 5, pp. 611-540, 2000 .

[29] G. Bansal and M. J. Hossain, "Optimal and suboptimal power allocation schemes for ofdm-based cognitive radio systems," IEEE Transactions on Wireless Communications, vol. 7 , no. 11, pp. 4710-4718, 2008.

[30] J. Mitola and G. Q. Maguire, "Cognitive radio: making software radios more personal," IEEE Pers. Commun., vol. 6, no. 4, pp. 13-18, 1999.

[31] S. Haykin, "Cognitive radio: brain-empowered wireless communications," vol. 23, no. 2, pp. 201-220, 2005.

[32] H. Tang, "Some physical layer issues of wide-band cognitive radio systems," Proc. IEEE Int. Symposium on New Frontiers in Dynamic Spectrum Access Networks, pp. 151-159, 2005.

[33] H. Urkowitz, "Energy detection of unknown deterministic signals," Proc. IEEE., vol. 55, no. 4, pp. 523-531, 1967.

[34] B. Wang and K. J. R. Liu, "Advances in cognitive radio networks: A survey," IEEE Journal of Selected Topics in Signal Processing, vol. 5, no. 1, pp. 5-23, 2011.

[35] I. F. Akyildiz, "Next generation dynamic spectrum access cognitive radio wireless networks: A survey," Computer Networks, vol. 50, no. 13, pp. 2127-2159, 2006.

[36] D. Huang, Z. Shen, C. Miao, and C. Leung, "Resource allocation in mu-ofdm cognitive radio systems with partial channel state information," EURASIP Journal on Wireless Communications and Networking, 2010.

[37] W. Yu and J. M. Cioffi, "On constant power water-filling," IEEE International Conference on Communications, pp. 1665-1669, 2001. 
[38] T. Yoo and A. Goldsmith, "Capacity and power allocation for fading mimo channels with channel estimation error," IEEE Transactions on Information Theory, vol. 52, no. 5, pp. 2203-2214, 2006.

[39] X. Kang, Y. C. Liang, A. Nallanathan, H. K. Garg, and R. Zhang, "Optimal power allocation for fading channels in cognitive radio networks: Ergodic capacity and outage capacity," IEEE Transactions on Wireless Communications., vol. 8, no. 2, pp. 940-950, 2009.

[40] Q. Qi, A. Minturn, and Y. Yang, "An efficient water-filling algorithm for power allocation in ofdm-based cognitive radio systems," International Conference on Systems and Informatics, pp. 2069-2073, 2012.

[41] D. Tse and P. Vishwanath, "Fundamentals of wireless communications," Cambridge University Press, 2005.

[42] P. S. Chow, J. M. Cioffi, and J. A. C. Bingham, "Practical discrete multitone transceiver loading algorithm for data transmission over spectrally shaped channels," IEEE Trans. Commun., vol. 43, no. 2-4, pp. 773-775, 1995.

[43] B. S. Krongold, K. Ramachandran, and D. L. Jones, "Computationally efficient optimal power allocation algorithm for multicarrier communication systems," IEEE Trans. Commun., vol. 48, pp. 23-27, 2000.

[44] N. Papandreou and T. Antonakopoulos, "A new computationally efficient discrete bitloading algorithm for dmt applications," IEEE Trans. Commun., vol. 53, no. 5, pp. 785$789,2000$.

[45] Z. Hasan and E. Hossain, "Energy-efficient power allocation in ofdm-based cognitive radio systems: A risk-return model," IEEE Transactions on Wireless Communications, vol. 8, no. 12, pp. 6078-6088, 2009. 
[46] G. Bansal, M. J. Hossain, and V. K. Bhargava, "Adaptive power loading for ofdm-based cognitive radio systems," IEEE International Conference on Communications (ICC), pp. 5137-5142, 2007.

[47] P. Wang, M. Zhao, L. Xiao, S. Zhou, and J. Wang, "Power allocation in ofdm-based cognitive radio systems," IEEE GLOBECOM, pp. 4061-4065, 2007.

[48] S. Barbarossa, S. Sardellitti, and G. Scutari, "Joint optimization of detection thresholds and power allocation for opportunistic access in multicarrier cognitive radio networks," IEEE International Workshop on Computational Advances in Multi-Sensor Adaptive Processing, pp. 404-407, 2009.

[49] Y. Zhang and C. Leung, "Resource allocation in an ofdm-based cognitive radio system," IEEE Trans. Commun., vol. 8, no. 7, pp. 1928-1931, 2009.

[50] E. Pei and S. Wang, "Energy-efficient power allocation for ofdm-based cognitive radio systems with imperfect spectrum sensing," Wireless Communications, Networking and Mobile Computing (WICOM), pp. 1-3, 2011.

[51] K. Son, B. C. Jung, S. Chong, and D. K. Sung, "Power allocation for ofdm-based cognitive radio systems under outage constraints," IEEE International Conference on Communications (ICC), pp. 1-5, 2010.

[52] X. Kang and Y. C. Liang, "Optimal power allocation strategies for fading cognitive radio channels with primary user outage constraint," IEEE Selected Areas in Communications., vol. 29, no. 2, pp. 374-383, 2010.

[53] T. L. Van, H. D. Chi, K. N. Viet, and H. N. Thanh, "Full-filling subcarrier power allocation in ofdma-based cognitive radio systems," Wireless Engineering and Technology, vol. 5, pp. 11-18, 2014. http://www.scirp.org/journal/wet.

[54] S. Srinivasa and S. A. Jafar, "Cognitive radio networks: how much spectrum sharing is optimal," IEEE Global Communications Conference (Globecom), pp. 3149-3153, 2007. 
[55] Q. Zhao, "Spectrum opportunity and interference constraint in opportunistic spectrum access," IEEE International Conference on Acoustics, Speech, and Signal Processing (ICASSP), pp. 1-6, 2007.

[56] W. Wang, T. Peng, and W. Wang, "Optimal power control under interference temperature constraints in cognitive radio network," IEEE Wireless Communications and Networking Conference (WCNC), pp. 116-120, 2007.

[57] K. Hamdi, W. Zhang, and K. B. Letaief, "Power control in cognitive radio systems based on spectrum sensing side information," IEEE International Conference on Communications (ICC), pp. 5161-5165, 2007.

[58] A. T. Hoang and Y.-C. Liang, "A two-phase channel and power allocation scheme for cognitive radio networks," IEEE PIMRC, pp. 1-5, 2006.

[59] Y. Xing, C. N. Mathur, M. A. Haleem, R. Chandramouli, and K. P. Subbalakshmi, "Dynamic spectrum access with qos and interference temperature constraints," IEEE Trans. Mobile Comp., vol. 6, no. 4, pp. 423-433, 2007.

[60] M. H. Islam, Y.-C. Liang, and A. T. Hoang, "Distributed power and admission control for cognitive radio networks using antenna arrays," IEEE DYSPAN, pp. 250-253, 2007.

[61] L. Le and E. Hossain, "Resource allocation for spectrum underlay in cognitive radio networks," IEEE Trans. Wireless Commun., vol. 7, no. 12, pp. 5306-5315, 2008.

[62] D. I. Kim, L. B. Le, and E. Hossain, "Joint rate and power allocation for cognitive radios in dynamic spectrum access environment," IEEE Transactions on Wireless Communications, vol. 7, no. 12, pp. 5517-5527, 2008.

[63] F. S. Chu and K. C. Chen, "Radio resource allocation in ofdma cognitive radio systems," IEEE 18th International Symposium on Digital Object Identifier, pp. 1-5, 2007. 
[64] S. M. Almalfouh and G. L. Stuber, "Interference aware subcarrier and power allocation in ofdma based cognitive radio networks," Multi-Carrier Systems and Solutions, vol. 60, no. 4, pp. 1699-1713, 2009.

[65] P. Zhang, L. Yang, and X. Liu, "Subcarrier and power allocation in ofdm-based cognitive radio systems," Computer Network and Information Security, pp. 24-30, 2010.

[66] T. Qin and C. Leung, "Fair adaptive resource allocation for multiuser ofdm cognitive radio systems," Second Int. Conf. Communications and Networking in China (CHINACOM), pp. 115-119, 2007.

[67] P. Cheng, Z. Zhang, H. Huang, and P. Qiu, "A distributed algorithm for optimal resource allocation in cognitive ofdma systems," IEEE International Conference on Communications (ICC), pp. 4718-4723, 2008.

[68] W. Yu and R. Lui, "Dual methods for nonconvex spectrum optimization of multicarrier systems," IEEE Transactions on Wireless Communcations, vol. 54, no. 7, pp. 1310$1322,2006$.

[69] D. T. Ngo, C. Tellambura, and H. H. Nguyen, "Resource allocation for ofdm-based cognitive radio multicast networks," IEEE Transaction on Vehicular Technology., pp. 1668$1679,2009$.

[70] "FCC Report of the Spectrum Efficiency Working Group," Federal Communications Commission Spectrum Policy Task Force, November 2002. 